\title{
Knowledge Flows between multinational Companies
}

Citation for published version (APA):

Schoenmakers, W. W. M. E. (2005). Knowledge Flows between multinational Companies. [Doctoral Thesis, Maastricht University]. Datawyse. https://doi.org/10.26481/dis.20050420ws

Document status and date:

Published: 01/01/2005

DOI:

10.26481/dis.20050420ws

Document Version:

Publisher's PDF, also known as Version of record

\section{Please check the document version of this publication:}

- A submitted manuscript is the version of the article upon submission and before peer-review. There can be important differences between the submitted version and the official published version of record.

People interested in the research are advised to contact the author for the final version of the publication, or visit the DOI to the publisher's website.

- The final author version and the galley proof are versions of the publication after peer review.

- The final published version features the final layout of the paper including the volume, issue and page numbers.

Link to publication

\footnotetext{
General rights rights.

- You may freely distribute the URL identifying the publication in the public portal. please follow below link for the End User Agreement:

www.umlib.nl/taverne-license

Take down policy

If you believe that this document breaches copyright please contact us at:

repository@maastrichtuniversity.nl

providing details and we will investigate your claim.
}

Copyright and moral rights for the publications made accessible in the public portal are retained by the authors and/or other copyright owners and it is a condition of accessing publications that users recognise and abide by the legal requirements associated with these

- Users may download and print one copy of any publication from the public portal for the purpose of private study or research.

- You may not further distribute the material or use it for any profit-making activity or commercial gain

If the publication is distributed under the terms of Article $25 \mathrm{fa}$ of the Dutch Copyright Act, indicated by the "Taverne" license above, 
Knowledge Flows between Multinational Companies

\author{
A Patent Data Analysis
}


Knowledge flows between multinational companies: a patent data analysis

(C) W.W.M.E. Schoenmakers, Maastricht 2005

Peefschrift Universiteit Maastricht

ISBN 9052784523

Omslag vormgeving: John Caris, Mastricht

Druk: Datawyse/Universitaire Pers Maastricht 


\title{
Knowledge Flows between Multinational Companies
}

\author{
A Patent Data Analysis
}

\section{PROEFSCHRIFT}

TER VERKRIJGING VAN DE GRAAD VAN DOCTOR AAN DE UNIVERSTTEIT MAASTRICHT, OP GEZAG VAN DE RECTOR MAGNIFICUS, Prof. Mr. G.P.M.F. MOLS

VOLGENS HET BESLUIT VAN HET COLLEGE VAN DECANEN, IN HET OPENBAAR TE VERDEDIGEN

OP WOENSDAG 20 APRIL 2005 OM 14.00 UUR

\author{
DOOR
}

Wilfred Wilhelmus Maria EduARdus SchoEnMakers 


\section{Promotoren:}

Prof. dr. J. Hagedoorn

Ptof. dr. G.M. Duysters

Beoordelingscommissie:

Prof. dr. ir. B.G.C. Dellaert (voorzitter)

Prof. dr. J.F. den Hertog

Prof. dr. A-P de Man (Technische Universiteit Eindhoven) 


\section{Acknowledgements}

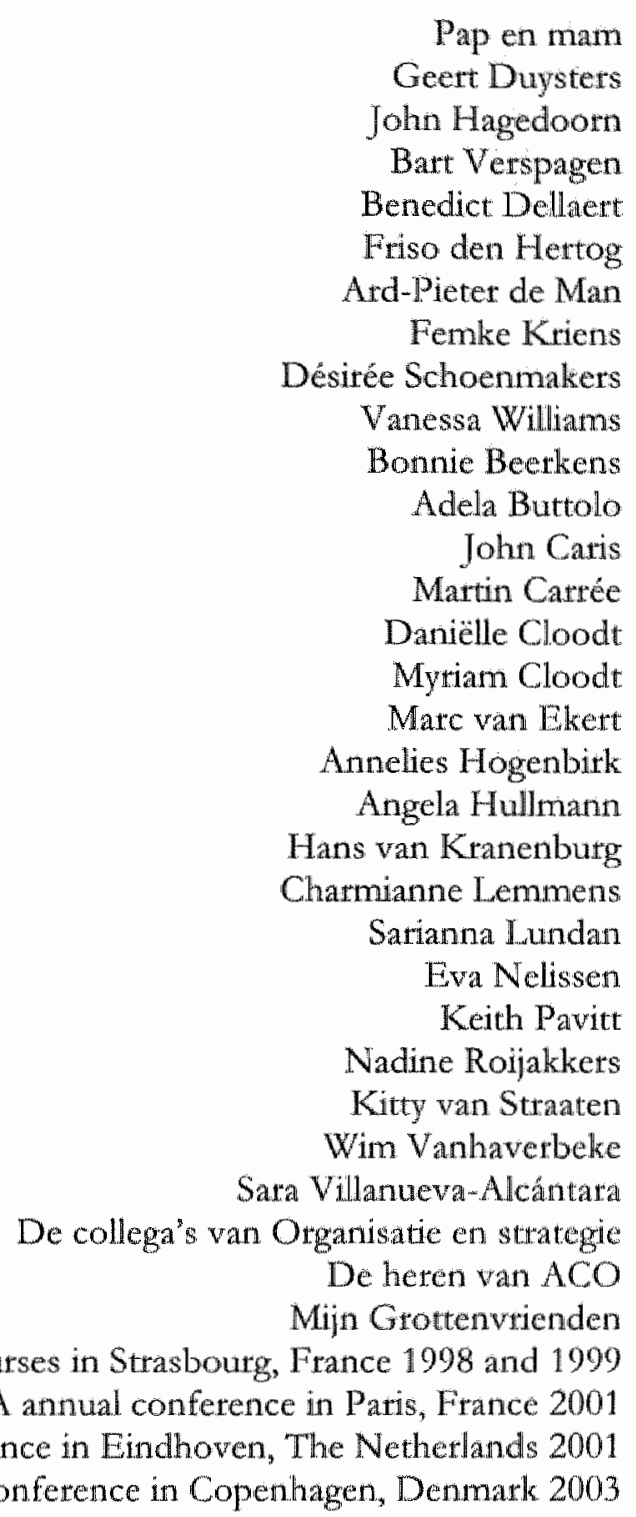

Participants at the ECIS conference in Eindhoven, The Netherlands 2001
Participants at the EIBA annual conference in Copenhagen, Denmark 2003

Participants of the ETIC Ph.D. Courses in Strasbourg, France 1998 and 1999

Participants at the EIBA annual conference in Paris, France 2001

Bedankt!

Wilfred Schoenmakers

Maastricht, 2005 



\section{Content}

Acknowledgements

I Introduction

1.1 Introduction

1.2 Theoretical background 12

1.3 Aim of the thesis $\quad 14$

1.4 Thesis outline 16

II Data, industry and sample

2.1 Introduction 19

2.2 Data sources and sample construction 19

2.3 Patent statistics and their characteristics 24

2.4 Sample choice $\quad 27$

III Factors influencing knowledge flows between multinational companies

3.1 Introduction 29

3.2 Theory and hypotheses $\quad 30$

3.3 Data 34

3.4 Methods $\quad 35$

3.5 Results 39

3.6 Discussion and conclusions $\quad 48$

IV Learning in strategic technology alliances

4.1 Introduction

4.2 Theory and hypotheses $\quad 52$

4.3 Data 58

4.4 Methods 59

4.5 Results 62

4.6 Discussion and conclusions $\quad 70$

V The spatial dimension of patenting by multinational firms in Europe

5.1 Introduction 73

5.2 Theory and hypotheses $\quad 74$

5.3 Data $\quad 78$

5.4 The spread of patenting over European regions $\quad 80$

5.5 The Blue Banana and beyond 85

5.6 Geographical distance and patent citations in Europe 90

5.7 Discussion and conclusions 101 
6.1 Introduction

6.2 Overall Summary and conclusions 103

6.2.1 Factors influencing knowledge flows 104

6.2.2 The influence of strategic technology alliances. $\quad 106$

6.2.3 The spatial dimension of knowledge flows $\quad 107$

6.2.4 Overall conclusions 110

6.3 Strengths and limitations, and suggestions, for future research

\section{Appendices}

A List of companies in the dataset

B Statistics of the firms in the dataset

\section{References}

Samenvatting (summary in Dutch)

Curriculum Vitae

\section{List of Tables}

Table 2.1 Number of firms per region

Table 2.2 Spread of multinational firms in the dataset over the European countries

Table 3.1 Means, standard deviations and correlations Dependent variable Cited and Citing

Table 3.2 Means, standard deviations and correlations for the dummy variables

Table 3.3 Tests for overdispersion in Poisson Model.

Dependent variable Cited

Table 3.4 Tests for overdispersion in Poisson Model.

Dependent variable Citing

Table 3.5 Results for the negative binomial regressions.

Dependent variable Cited

Table 3.6 Results for the negative binomial regressions.

Dependent variable Citing

Table 4.1 Descriptive statistics for the variables in hypotheses 1 and 3

Table 4.2 Correlations for the variables in hypotheses 1 and $3 \quad 64$

Table 4.3 Results Tobit regression hypotheses 1 and $3 \quad 68$

Table 4.4 Results t-test and Willcoxon Signed Ranks test 69

Table 4.5 Regression results hypothesis 2

Table 5.1 Share of patents invented in foreign regions in Europe by European based MNEs, 1997 
Table 5.2 The number of citation links with positive citations, within and between MNE groups, by sector, cited patents in 1994, citing patents 1994-1997

\section{List of Figures}

Figure 2.1 Number of patents per year for the whole dataset 23

Figure 2.2 Citation-lag for the patents in the whole dataset 24

Figure 3.1 Number of patents per year for the whole dataset 35

Figure 3.2 Citation-lag for the patents in the whole dataset 36

Figure 4.1 Spread of the dependent variable 63

Figure 4.2 Gross learning effect $\quad 65$

Figure 4.3 Net learning effect 66

Figure 5.1 The presence of large MNEs R\&D activities in European regions $\quad 82$

Figure 5.2 The number of patents vs. the average spread of patenting over European tegions, firm level 85

Figure 5.3 The Blue Banana and beyond 87

Figure 5.4 Patenting activity by all firms in our dataset $\quad 89$

Figure 5.5 Citation patterns by sector and for within and between MNEs citations 95

Figure 5.6 The impact of distance and time on patent citations, within and between MNEs, chemicals

Figure 5.7 The impact of distance and time on patent citations, within and between MNEs, electronics and motor vehicles 



\section{CHAPTER 1 \\ Introduction}

\subsection{Introduction}

The notion in modem day economics of technological progress through innovation and knowledge creation as instrument for economic growth (Cohen and Levinthal 1989; Kogut and Zander 1992; Nonaka, Byosiere et al 1997; Teece, Pisano et al 1997; Edmondson, Bohmer et al. 2001; Meyer 2002) is not new. Already Adam Smith acknowledged the importance of innovation for the growth of the economy as a whole and for individual companies in his seminal work "The Wealth of Nations" (Smith 1776). Adam Smith considers the division of labour and the "improvements in machinery" as important contribuants to a firm's, as well as a country's wealth. Also Karl Marx recognized the significance of inventions and innovations as a very important element for the growth of economies (Marx 1858). "...the bourgeoisie cannot exist without constantly revolutionizing the means of production". Alfred Marshall as well considered (scientific) knowledge to be an important contribuant to overall wealth (Marshall 1936).

The prosperity experienced by many ancient civilisations and also our own are for a large part due to the development and dissemination of knowledge and innovations. The rise of for instance the Roman Empire can be attributed to technological progress made in Roman society, for instance in the fields of iron making. The wealth we know of as today in our western world comes from deliberate and coincidental technological improvements and knowledge generation. Besides major inventions, like for instance the steam engine transistor and the personal computer, the small incremental innovations as well are responsible for much of the experienced growth in the economy (Smith 1776; Freeman and Soete 1997).

In this chapter we will give a short introduction into the main theories used in this thesis. We will explain the aim of the thesis and provide the main research question as also the three specific research questions that will be answered in the empirical chapters. We will discuss the main contribution of the thesis, and finally will present an outline of the thesis. 


\subsection{Theoretical background}

Joseph Schumpeter laid down the foundations for modern day economic theories on innovation (Schumpeter 1934; Schumpeter 1939; Schumpeter 1942). According to Schumpeter (Schumpeter Mark I) innovation was the main driving force behind capitalist growth and was a main source of company profits. Schumpeter postulated that people and companies have incentives to innovate because innovations can help them achieve a profitable market position, which provides them a temporary monopoly. People and companies combine the publicly available knowledge to develop new knowledge, with which they than can challenge existing firms in a market niche. Successful firms in this way can gain a temporarily monopoly position. Howvever other innovating firms will in tum challenge their position and their knowledge will leak away to these other firms. This mechanism will lead to cycles of, as Schumpeter calls this, "creative destruction". This view would favour the small innovating firms and lead to a more competitive market environment. Because of the leaking of knowledge, knowledge spillovers, without suitable appropriability, firms in this context will not have an incentive to innovate once they have achieved a leading edge.

On the other hand Schumpeter also argues that innovations might be cumulative (Schumpeter Mark II). This would favour large established firms with a long innovation history. Firms would build their innovations on their earlier innovations, and be able to appropriate a large part of it. This is labelled. by Schumpeter "creative accumulation" and would lead to a highly concentrated market. The level of appropriability can be high, since firms can for instance use patents to protect their innovations and on the other hand their innovations are difficult to replicate or imitate because they are cumulative. Therefore firms in this environment are stimulated to keep on innovating, to stay ahead.

Another theory, which incorporates the influence of innovation on the economy, is the New Growth theory, which emerged in the 1980s (Romer 1986; Lucas 1988; Romer 1990) due to a renewed emphasis on information, knowledge and skills. This theory placed technological change in the heart of economic theory by the assumption that knowledge is endogenous to the production process. The focus is on the knowledge externalities brought about by the publicly availability of a large part of the knowledge. In this way not only do the benefits of a firm's newly created knowledge accrue to the firm itself, but also to the economy at large. Thus the development of knowledge within one firm may, because of the knowledge externalities, generate new knowledge in other firms. A distinct characteristic of the New Growth theory is the implication that knowledge might be spatially bounded, which could explain the differences in growth rates between areas. 


\section{NTRODUCTION}

All these theories concentrate for the most patt on the influence of knowledge and innovation on the economy as a whole, and are concerned with undelibetate knowledge exchange, knowledge spillovers. They are less concerned with the influence of knowledge and innovation on the firm level, and forgo the deliberate knowledge exchange that we experience in modern day economic environment. However important, both for the economy as a whole and for the individual company's success, knowledge spillovers alone are not enough in moden day high- and medium-tech sectors of the economy to survive. Picking up more or less free knowledge from the environment by itself is not enough to succeed and is also increasingly more difficult to do. On the one hand has knowledge become much more idiosyncratic, and is thus more and more difficult to implement in the own firm situation. On the other hand has the technology used by companies taken such a flight that companies most often cannot go it all alone. Firms need to work together and exchange each other's knowledge.

During the second half of the twentieth century we see major developments in the field of strategy. Here the focus is more on the individual firm level and less on the economy as a whole. Also do we see increased emphasis here on deliberate knowledge transfer between companies as part of an organisational learning strategy.

One of the most noteworthy theories of the firm is the resource-based view of the firm. This theory finds its origins in the work of Penrose (1959) and Wernerfelt (1984), who were followed by many others (e.g. Prahalad and Hamel 1990; Barney 1991; Kogut and Zander 1992; Peteraf 1993; Rumelt 1996). A core feature of this theory is the supposed difference across firms in the way they control strategically important resources, and the presumed stability of this difference; organisational learning is in this theory thus not considered. Firms are furthermore believed to seek to increase their economic performance, and the difference berween firms" resource endowment is held responsible for the performance differences between firms. Firm resources are considered valuable, rare and difficult to imitate or substitute. Firms are thus successful according to the resources they posses, and the value these resources can deliver for them. The spectrum of resources a firm controls at a certain moment in time is stable and difficult to adjust or expand.

Out of the resource-based theory of the firm emerged another theory of the firm, the dynamic capabilities view of the firm (Prahalad and Hamel 1990; Nelson 1991; Teece, Pisano et al. 1997). In this theory, not the exploitation of current resources is of fundamental importance, but rather the alteration in the bundle of a firm's resources. The ability to create novel resources for the firm is considered the most important tesource of all. Firm performance differences thus occur because of differences in the ability of firms to build new resources, which is by itself a core tesource. In this theory organisational learning is a 
related and importart concept (Teece, Pisano et al. 1997). Only via organisational learning, be it internal ot external, will firms be able to change their current resources, or deliver new ones.

A second successor of the resource-based theory of the firm is the knowledge-based theory of the firm (Kogut and Zander 1992; Grant and Baden-Fuller 1995; Foss 1996; Grant 1996). This theory also concentrates on the dynamic nature of firm resources, so also in this theory organisational learning is considered very important. The core resource in this theory however is the knowledge residing within the firm, be it codified knowledge contaned in patents of copyrights, or tacit knowledge, which tesides in the employers minds and routines. Next to considering organisational learning this theory also delivers a valuable explanation for the increased collaboration between firms, for one of the reasons why companies cooperate might be that they want to learn from each other (Hagedoom 1993b). Firm performance differences are thus explained by the way firms manage their in-house knowledge and succeed in incorporating external knowledge with their internal knowledge.

Since organisational learning, and thus knowledge flows turn out to be so important for individual company success, it would be interesting to investigate this relationship further. The focus of out study is therefore on the individual firm level, were we use the theories of the firm as an important theoretical tool for explaining the observed phenomena of organisational learning and knowledge flows.

\subsection{Aim of the Thesis}

The purpose of this thesis is to enlighten the knowledge flows that occur between multinational companies, Understanding the nature and direction of knowledge flows can aid us in comprehending how organisations learn, which, as discussed before, is important in explaining firm performance differences (Cohen and Levinthal 1989; Kogut and Zander 1992; Nonaka, Byosiere et al 1997; Teece, Pisano et al 1997; Edmondson, Bohmer et al. 2001).

Out main reseatch question is therefore: Wbat is the natwe of kmowledge fows betwen mulhinational companies? To answer this central research question we should answer sevetal more specific research questions. For our understanding of the knowledge flows it is important to start by researching the factors that influence these knowledge flows. In the literatute find much research devoted to this topic Nadiri 1993; Appleyard 1996; Blomstrom and Sjoholm 1999; Gupta and Govindarajan 2000; Jaffe, Trajtenberg et al. 2000a; Plasmans and Lukach 2001; Steenhuis and De Bruijn 2002; Stel and Nieuwenhuijsen 2002; Monjon and Waelbroeck 2003), but most researches are concentrated only on one part of the knowledge flows, the undeliberate knowledge spillovers 
and leave out the deliberate part of the knowledge flows. Most researchers however agree on a direct relationship between knowledge flows and patents, and between knowledge flows and R\&D expenses (Pakes and Griliches 1980; Griliches 1984; Dumont and Tsakanikas 2001; Cohen, Goto et al. 2002). We research both the deliberate and the undeliberate knowledge flows and also study the influencing factors jointly. We are thus interested in the characteristics of the knowledge flows, so what factors influence knowledge flows and in what direction?

Besides positive results for the number of patents and the $R \& D$ expenses we also find positive influences in the literatuze for strategic alliances on knowledge flows (Hagedoorn 1.993a; Mody 1993; Mowery, Oxley et al. 1996; Inkpen 1998; Larsson, Bengtsson et al. 1998; Hagedoorn and Duysters 1999; Parise and Henderson 2001). Usually these results are however based on small datasets, and datasets focused only at the multinational headquatters. Our aim is it to investigate this relationship in a broad and comprehensive manner. We want to include all large multinational firms in the high- and medium-tech sectors of the economy, and look not only at the multinational headquarter but instead look at the whole multinational "group", so inclusive all subsidiaries. Besides investigating the influence of strategic alliances on the knowledge flows as a single factor we also want to investigate the influence of strategic alliances on organisational learning in-depth. Strategic alliances are considered to be important for organisational learning (Hagedoom 1993b; Mody 1993; Inkpen 1998; Khanna, Gulati et al. 1998; Mowery, Oxley et al. 2002). We are thus interested to know what the influence is of strategic technology alliances on knowledge flows between allying firms? We also want to know if allying firms for instance learn better than non-allying firms?

As a last research question we are interested in the spread of knowledge over geographical areas. Since we first investigated the factors influencing the knowledge flows, and took a closer look at the influence of strategic technology alliances, it is now time to turn to the observed spread of the knowledge itself. Knowing how knowledge flows in geographical space is important: for firms that are interested in learning from the environment, and for governments that want to stimulate organisational learning in their region. One might think that advances in communication technology eradicated distance, as some authors do (Morgan 2004). We do find however that much of the MNEs R\&D activities are done in the home country, but that another non-negliable part of the R\&D activities is done abroad (Patel and Pavitt 1991; Le Bas and Sierra 2002). It would thus be interesting to investigate this second part further, and to compare our results for the spread of innovative activity across Europe with other researches in this field (e.g. Cancwell and Iammarino 2001; Hospers 2003). Other researches have studied this subject before, but never on a scale like we do in our research and these researches did not use firm level data as we 
ate doing (Caniëls 1999; Breschi 2000; Maurseth and Verspagen 2002). To our knowledge we are presenting the first European regional analysis of patenting by the world's largest companies, based on European patents. Using our extensive database we are now able to test relationships that could not be tested before. We are thus interested to know if knowledge flows are spatially concentrated or not, and we want to know if knowledge flows freely actoss geographical boundaries or not?

Our main research question can thus be covered by three specific research questions that make up our three empirical chapters:

- What factors influence knowledge flows between multinational companies, and what is the direction of their influence?

- What is the influence of strategic technology alliances on knowledge flows between multinational companies?

- Are knowledge flows spatially concentrated and spatially bounded?

The main contribution of this thesis lies in the fact that it researches the knowledge flows between multinational companies on the basis of a very extensive database. This is the first time ever that the knowledge flows between multinational companies have been empirically tested on such a scale and with such a degree of specificity. For this reason we needed to construct our own dataset, which is described in chapter 2 . The database is extensive in the sense that not only virtually all large multinational companies operating in the medium- and high-tech sectors are included in our analysis, we also constructed the dataset in such a way that all the multinational companies' patents are aggregated under the "parent" firm. In this way we get a better coverage of the

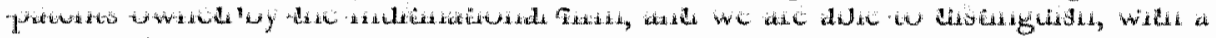
high degree of accuracy, between intra-firm and inter-firm knowledge flows, making our data set very precise. We furthermore were able to combine two extensive databases, one on patents (the EPO database) and one on alliances (the MERIT-CATI database), into one overall database. The combining of these databases gives our database research possibilities that were not available before on this scale. As a last point we want to mention the use of the European Patent office database in this thesis, which makes the thesis a valuable supplement to existing research, based on US patent data.

\subsection{Thesis outline}

The outline of the thesis is as follows. The main body of the thesis consists of one descriptive chapter and three empiricall chapters. Chapter 2 


\section{INTRODUCTION}

describes the extensive database construction process of the final database that we will use throughout this thesis. In chapter 2 we also explain the chatacteristics of the different databases we used to construct our final database. We explore the limitations and shortcomings of the data, and justify the selection of the sample we use.

Chapter 3 is the first empirical chapter, where we investigate the factors influencing the knowledge flows between multinational companies. Based on the database constructed as described in chapter 2 we test what factors influence the knowledge flows to and from the multinational companies in our database, and determine the direction of the influence.

In chapter 4 we investigate the influence of strategic technology alliances on the knowledge flows between multinational firms. By combining our own database with the MERIT-CATI database we tested in what way strategic technology alliances play a role in knowledge flows, and looked at the nature of the learning within strategic technology alliances.

Chapter 5, our last empirical chapter investigates how spatially bounded knowledge is. On the one hand do we want to test how spatially concentrated knowledge flows are; on the other hand are we interested in the spatial diffusion pattern of knowledge. By dividing Europe into different "NUTS" regions we tested our assumptions on the geographic characteristics of knowledge.

Chapter 6 is our concluding chapter where we summarise the results from our three empirical chapters and answer the main research question. We will also highlight the strengths and limitations of our research and give directions for further research. 


\section{CHAPTER 2 \\ Data, Industry and Sample}

\subsection{Introduction}

In this chapter we will explain the database construction of the data set that we will be using throughout the rest of this book. We will indicate the advantages and the disadvantages of the data that we use, especially the patent and patent citation data, and we will explain the reasons for the selection of multinational firms in the medium-and high-tech sectors in our data set.

\subsection{Data sources and sample construction}

For our research we made use of several different databases, which we used to construct our own database. We started off with the Fortune 500 list with the 500 largest companies in the world. From this list we selected all the firms that were in high- and medium-tech sectors. The reasons for selecting these sectors will be discussed below. This left us with 171 multinational firms. We made further use of two other databases, namely the EPO (European Patent Office) database on patents and the Dun \& Bradstreet linkages database on parent-subsidiary relationships between firms.

Our primary data source is the EPO database on patent applications. We selected all patent applications, whether they were granted, have been rejected (or withdrawn), or are still under review. The EPO database we use contains data from 1978 up to the beginning of 1999 , including approximately 935,000 patent applications. Unfortunately, for the purpose of identifying within-firm patent applications, we cannot rely upon the information that EPO supplies in the "applicant name" field. In that field, one may find personal names or names of firms or organizations. In the case of firms, however, it may be the name of an independent firm, or the name of some form of a larger conglomerate or holding firm. The EPO database contains approximately 180,000 unique names in the "applicants" field.

Our sample of firms is limited to large multinational firms that appeared on the Fortune 500 list in 1997, supplemented by a few large firms from the Fortune lists in earlier years. For these firms, we made use of the Dun \& Bradstreet Linkages database to construct a list of the subsidiaries. "The Dun \& Bradstreet Linkages database includes only full, i.e, one hundred percent, 


\section{CHAPTER II}

subsidiaries. We refer to this list as the "group". "The version of the Dun \& Bradstreet Linkages database we used is from late 1998, and represents thus the parent-subsidiary relationships at that point in time. Of course these relations have not atways been like they were in 1998. Thus, when we use the parentsubsidiary ties of 1998 to construct patent data for groups during the petiod 1976-1998, the resulting data does not reflect gtoup patent data at the time of the patent applications involved, but rather at some ex-post period in time (1998). Obviously, this is a sub-optimal procedure. A better procedure would be to use yearly data from the Dun \& Bradstreet Linkages database, and to construct for every year under investigation a groups database as we did for 1998. "The construction of these "group" lists for other years is however still under construction.

There are, nevertheless, at least two reasons why we feel that the procedure used is acceptable for the present purposes. First, most multinational companies apply for the bulk of their patents undet the multinational company name. In this area the changes are less pressing, i.e., mergers occur more often on a lower level. Second, the main aim of using group level data is to exchude intra-firm patents. Although one cannot rule out mistakes on this part that result from the present procedure, it seems reasonable to expect that the number of such mistakes is relatively small. Such a mistake would result if a firm would patent during the period under consideration through a certain subsidiary, and sell off this subsidiary before 1998 (our point of reference for linkages). Given the tendency to patent through group headquarters, one would expect such patents to be relatively small in number.

A further practical problem results from the fact that there is not a oneon-one correspondence between the subsidiary names it the Dun \& Bradstreet Linkages database and the names in the "applicant" field of the EPO database. We made a preselection from the EPO database by searching for different parts of the names found in the linkages data. The results from this preselection were then compared, usually on ane-on-one basis, to the group list ftom Linkages. Some names that were found in the pre-selection from the EPO database could not be identified using the D\&B database. In many of these cases, the name found in the EPO database was partly identical to the name of the (subsidiary) firm we were looking for. This may, for example, happen if the applicant name is the name of a platt, rather than the legal entity it belongs to. In order to be able to learn more about these firms, we constucted a table with the applicant names and addresses from the EPO database. In this way we could compare not only the applicant's name but also the address to the data found in Linkages. If the applicant was not found in the D\&B database, but the applicant's name was almost identical and the address was identical to another subsidiary firm of the same multinational, then we included the name in the group list. 
A final note refers to the case when companies merge, and the original company name under which they applied for a patent might be lost. This could mean that we would not find these patents, although they belong to the multinational firm under investigation. Therefore we also looked at different parts of the company name, thereby eliminating as much as possible this bias. We also made use of the historical information given by the multinationals on their Intemet pages and available in the D\&B database to search for patents applied for under an earlier company name.

From the EPO database we also extracted the patent citation information, which comes with every patent. This supplied us with a table with two columns where the first is the cited patent, and the second is the citing patent, both with their application date. In this way it is possible to follow the "paper trail" of a patent, to designate the importance of a patent based on the number of citations it has, and to contrive the citation-lag of the patents (Carpenter, Narin et al. 1981; Albert, Avery et al. 1991; Jaffe, Trajtenberg et al. 1993). Following Jaffe et al. (1993) we take a patent citation to be evidence of a subsequent technological development building on the result of the cited patent.

For the data on the amounts spend on R\&D, the number of employees per firm, and the revenues per firm we made use of the "Worldscope" database, and the Fortune Global 500 list for 1998. All money values were converted to US dollars to facilitate comparison.

The data about the alliances of the companies was taken from the MERIT- CATI (Cooperative Agreements and Technology Indicators) database (see Hagedoorn 1993b). The CATI database is a relational database containing information on the number and sort of over 15,000 cooperative agreements involving about 9500 firms, and over 3500 different parent companies. Systematic collection of inter-firm cooperation's started in 1987, but earlier years were searched in retrospect. Different sources were used for the construction of the database, among the most important are newspapers and trade journal articles. Even though the dataset will inevitably be incomplete, and biases might be present, CATT is the most complete and dependable source available on cooperative agreements. The CATT database contains only information related to technological cooperation, so those agreements where a combined innovarive activity or an exchange of technology is at least part of the agreement. Partnerships that regulate no more than the sharing of production facilities, the setting of standards, collusive behaviour in price setting and the raising of entry barriers are omitted from the database.

We ended up with information on 171 multinational firms ${ }^{1}$ operating in the following twelve sectors (numbers in brackets refer to the number of firms

\footnotetext{
${ }^{2}$ For the statistics of the firms in our dataset see appendix $B$.
} 
per sector): Aerospace (8); Chemicals (16); Computers (8); Electronics (25); Semiconductors (2); Industrial and farm equipment (8); Metals (13); Motor vehicles (25); Petroleum refining (31); Pharmaceuticals (10); Scientific, Photo, Control equipment (3); and Telecommunications (22). An alphabetical list of all firms can be found in appendix $A$.

All the firms are multinational firms of whom the headquarters are located all over the world with a main focus on the Triad USA, Europe and Japan, see table 2.1. Of all the firms in our daraset $35 \%$ has the headquarters located in the USA, 34\% has its headquarters in Europe, and for $23 \%$ of our firms their headquatters is located in Japan. $92 \%$ of the firms in our dataset thus have a headquarters located in the Triad countries.

Table 2.1; Number of frrms per region.

\begin{tabular}{|l|c|}
\hline Region & Number of firms \\
\hline USA & 60 \\
\hline Europe & 58 \\
\hline Japan & 39 \\
\hline Other & 14 \\
\hline
\end{tabular}

The multinationals with a home base in Europe are spread over eleven countries (sce table 2.2) with a main focus on Germany, England and France. Of the "other" countries South Korea, with 6 out of 14 , has the most multinational headquarters within its borders.

Table 2.2: Spread of multinational firms in the dataset over the European countries.

\begin{tabular}{|c|c|}
\hline European Country & Number of firms in the dataset \\
\hline Germany & 16 \\
\hline Ingland Feance & 10 \\
\hline Iraly & 5 \\
\hline Netherlands $\backslash$ Switzerland. & 4 \\
\hline Sweden & 3 \\
\hline Nonway spain & 2 \\
\hline Belgium Finland & 1 \\
\hline
\end{tabular}

If we take a closer look at the patents in our dataset (see figure 2.1) we see that the graph starts in 1978. From 1978 onwards we see the number of patents applied for increase steeply, till about 1990. After 1990 this increase in patent applications comes to a hold, and we even observe a slight slowdown in the number of patent applicarions, although not substantial. The number of patent applications stays more or less constant until 1997; our last data point. An explanation for the observed pattern can be found in the opening of the EPO in June 1978, following the signing of the European Patent Convention in 
1973, which came in to force on October 1977. The first applications were received on 1 June 1978. The steep increase from 1978 till 1990 is very probably a consequence of the firms having to get acquainted with the EPO. If firms judge applying with the EPO as a valuable protection for their inventions they will make more use of patenting in later periods. Also firms that see the successful patenting of other firm will start using patenting to protect their inventions. This will go on till a certain saturation point is accomplished, for our data apparently around 1990.

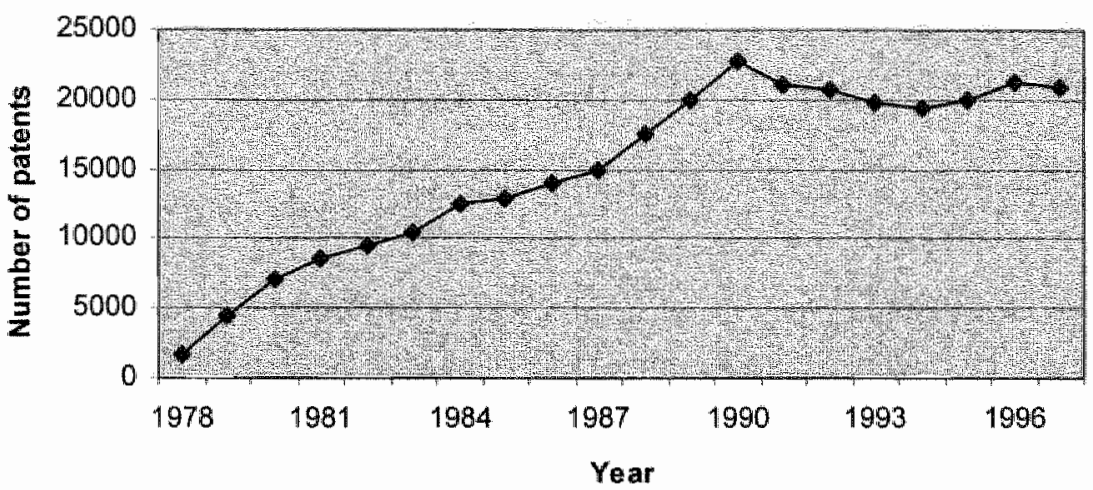

Figure 2.1: Number of patents per year

For the patents in our dataset we also plotted their citation-lag. This is the number of patents per year, after the original patent application, that cited our original patent. The citation-lag for our patents can be seen in figure 2.2 . We see that there is a sharp increase for the first 3 years after the original patent application, after which the citations gradually decline to zero. After about twenty years after the original patent application there are no significant patent citations for the patents in our dataset. This is inline with other research (Jaffe and Trajtenberg 1998). Patents are most interesting to cite just after their application, for the knowledge included in the patent is new at that time and thus valuable to other firms. When time passes the knowledge in the patents gets outdated and replaced by new, more valuable knowledge in younger patents. Only very important robust patents survive, but also their value will deteriorate over time, till eventually after about twenty years their vallue is close 


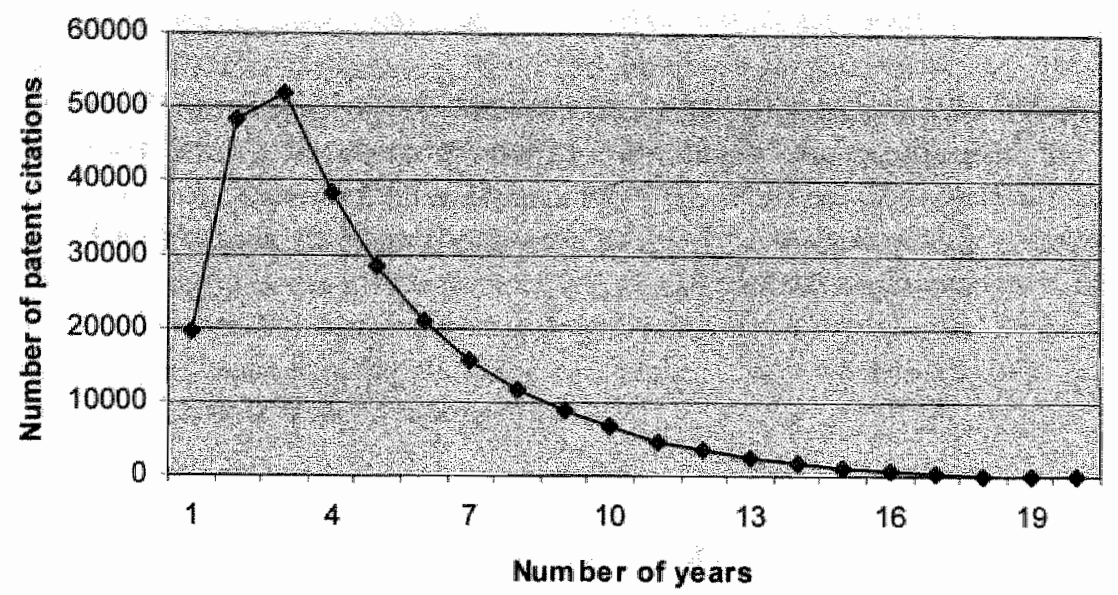

Figure 2.2: Citation-lag for the patents in the whole dataset.

to zero. Most patent citations occur however within four to five years after the original patent application, since the knowledge contained in the patents is than most valuable to other firms.

\subsection{Patent statistics and their characteristics}

Patents are intended to raise the incentive to innovate. Furthermore they can provide a useful indicator for technology trends. A patent is a legal title granting its owner the exclusive right to make use of an invention for a limited area and time by prohibiting others from, amongst orher things, making, using or selling it without authorisation (European Patent Office 2004). They thus confer a legal and enforceable monopoly on the patent holder for a certain period of time, and for a certain area (Parker 1978). The time span of a patent is typically twenty years, and the area is usually confined to the country where the patent is applied, or as in case with the European Patent Office, all the member states of the EPO (at the moment there are 26 member states). A patent is a property right on the commercial use of an appliance; ideas or computer programs are for instance not patentable (Parker 1978; Jaffe, Trajtenberg et al. 1993; Cook 2002). For a patent to be granted, the knowledge contained in it must be non-trivial, and useful. This means the new knowledge must be a novel and useful contribution to the existing field of knowledge. It must not be an obvious invention to experts in the field and it must have potential commercial 
significance (Michel and Bettels 2001). If the new knowledge has these properties than a legal title will be created containing information on for instance the name of the inventor, the employer of the inventor and the technological antecedents of the new knowledge; the patent citations. In the EPO system the applicant can include patent citations, but ultimately the patent examiner from the patent office determines what citations will be included. These patent citations can tell us something about where the knowledge contained in the patent comes from, and thus in a way form the paper trail of the knowledge ancestry of the patent (Jaffe, Trajtenberg et al. 1993). The patent applicant, in the EPO system, may supply information about prior art, known to him, but ultimately the patent examinet, being an expert in the distinct technological field, is supposed to identify all the relevant prior art (Michel and Bettels 2001).

The most obvious advantage of patents is their easily and abundant availability at relatively low cost. They hold tremendous amounts of information about the invention, the inventor and his employer, for very latge time series. Patents are held by many firms and virtually all research organisations, and ate publicly available in computerised form. Not only do patents have a much broader coverage than any other form of data, the information contained by the patents is also very extensive. Besides information on the knowledge lineage, there is information on the knowledge classification, the date of application, the addresses of the inventor and the organisation that holds the patent right. The combination of geographical information with citation information, both contained by the patent, provide for instance a unique way of tracing knowledge flows across time, space and different organisations (Jaffe, Trajtenberg et al. 1993), as can be seen in chapter five.

Besides adwantages, patent statistics also have disadvantages (Griliches 1984; Pavitt 1988; Archibugi 1992; Griliches 1998; Jaffe and Trajtënberg 2002). One of the most obvious disadvantages is the fact that many inventions are never patented. Besides that much research output cannot be patented, firms might also choose not to patent and instead rely on secrecy to protect their new knowledge (Jaffe, Trajtenberg et al. 1993). Patenting can sometimes be considered a strategic decision. Another critique concentrates on the differences in patenting behaviour between sectors (Levin, Klevorick et al. 1987; Griliches 1998). Some sectors rely heavily on patenting to protect their intellectual property where others do not. $A$ third important critique concerns the equal weight given to all patents, distegarding the importance of some very significant patents (Griliches 1984; Griliches 1998). A last critique involves the fact that patents only cover codified knowledge, and neglect the tacit component of knowledge. This problem is especially pressing since a high proportion of firm capabilities are tacit (Arora 1995; Patel and Pavitt 1997). 
Despite these shortcomings of patent data, many researchers consider patents to be a valuable and useful indicator of firm performance differences in technology (Griliches 1984; Basberg 1987; Acs and Audretsch 1988; Pavitt 1988; Napolitano and Sirlli 1990; Freeman and Soete 1997; Griliches 1998; Patel 2000; Acs, Anselin et al. 2002). Most of the problem associated with patent statistics can also be solved one way or the other by appropriate sample construction. The chances of firms patenting their innovations increases for instance with firm size (Scherer 1965; Archibugi and Pianta 1996). Also from empirical research we know that a large part of firms" inventions are patented, again this increases with firm size (Mansfield 1986; Arundel and Kabla 1998). The differences in sector patenting can be solved by sticking to only one sector or by using sector dummies (Griliches 1998). In making use of patent citations we can also distinguish between important en less important patents. We can expect important patents to have consiclerably more citations than their less important counterparts (Griliches 1998). Lastly the fact that patents do not cover the tacit component of a knowledge flow is considered to be not an important problem, since tacit and codified knowledge are considered to be two forms of knowledge that are complementary, not substitutes (Patel and Pavitt 1997).

Patent citations are more and more used in research next to simple patent counts. As noted before patent citations can give us an indication of the importance of a patent. Furthermore via patent citations we are able to follow the knowledge flow from one patent to the other. We follow the reasoning of Jaffe and Trajtenberg (1998) that patents are a proxy for "bits of knowledge" and patent citations are a proxy for the usefulness of a given bit of knowledge in de development of a descendant bit. Patent citations are also more and more used and approved of in different research areas (Carpenter, Narin et al. 1981; Carpenter and Narin 1983; Narin, Noma et al. 1987; Pavitt 1988; Albert, Avery ct al. 1991; Jaffe, Trajtenberg et al. 1993). Patent citations have the same advantages and suffer from the same disadvantages as patents daffe and Trajtenberg 1998; Hall, Jaffe et al. 2001); these will not be reported here again. Two extra problems occur with patent citations because some knowledge will flow between companies without being incorporated in a patent citation, while other patent citations might not indicate a true knowledge flow. Jaffe et al. (2000a) presented empirical evidence for this relationship, which led them to conclude that patent citations are a "noisy" indicator of knowledge flows. Nevertheless patent citations are considered to offer a valuable indicator for the knowledge flows between multinational companies, and are therefore useful for our research Jaffe, Trajtenberg et al. 1993; Griliches 1998; Jaffe and Trajtenberg 1998; Jaffe, Trajtenberg et al. 2000a). 


\subsection{Sample choice}

For our research we selected only multinational firms that are operating in medium- or high-tech sectors. There are several teasons for choosing these firms and these sectors. Since we are interested in knowledge flows, and are trying to measure knowledge flows using patent statistics, we needed to select secrors with a high patenting propensity. Low-tech sectors are by definition not very patent active, and would thus not suffice for our research ${ }^{2}$. Furthermore, although some researchers are critical about the use of patent statistics, in general most researchers agree that they are a useful indicator in medium- and high-tech sectors (Mansfield 1986; Arundel and Kabla 1998).

The reason for choosing only multinational firms is that these firms tend to be very active on different technological fields, and that the chances of firms patenting increase with firm size (Scherer 1965; Arundel and Kabla 1998). Small firms also relay more often than latge firms on secrecy instead of patenting, probably due to their lesser power to fight patent infringement (Arundel 2001). By choosing only multinational firms we thus also embark upon the probiem of patent differences between large and small firms, as noted before.

Furthermore, alliances are very well documented for multinational firms, something that is not the case for smaller firms. Also other data, like R\&D spending and number of employees are much better available for multinational firms than for smaller firms. Multinational firms also suffer to a lesser extent from most of the shortcomings encountered when using patent statistics as in the case of smaller firms (Griliches 1998).

2 The OECD identifies high-, medium- and low-tech sectors based on their R\&D intensity 
$\therefore$ 


\section{CHAPTER 3 \\ Factors Influencing Knowledge Flows between Multinational Companies}

\subsection{Introduction}

Performance differences between firms can be explained using the resource-based view of the firm, were company resources or capabilities are considered essential for company success (Penrose 1959; Wernerfelt 1984; Prahalad and Hamel 1990; Barney 1991; Peteraf 1993). Another theory that offers an explanation for differences in firm performances is the dynamic capabilities view of the firm (Nelson 1991; Teece, Pisano et al. 1997). This theory can be considered to be a special case of the resource-based wiew. According to the dynamic capabilities view, differences in firm performance occur because of differences in the renewal capability for firm capabilities. For this theory learning, internal learning as well as inter-organisational learning, is considered to be important. This implies that knowledge flowing to and from the company is critical in explaining company performance. Especially in turbulent economic environments does this last theory appear to be better suited for explaining performance differences between companies.

Since knowledge flows turn out to be of such crucial importance, it would be interesting to investigate the factors that influence the knowledge flows to and from a company. Knowing these factors can aid us in gaining a better understanding of the knowledge flows, and thus of the organisational leaming, which in turn could support us in understanding the reasons for differences in firm growith.

Following Jaffe and Trajtenberg (1998) we use patents as a proxy for knowledge "bits", and patent citations as a substitute for a knowledge flow. Based on the concept of absorptive capacity (Cohen and Levinthal 1990), and internal as well as external learning via R\&D investments and alliance formation, we investigate the main factors influencing the knowledge flows to and from the company. Using a database of 171 multinational firms in the medium- and high-tech sectors we test our hyporheses using negative-binomial regression. 


\subsection{Theory and hypotheses}

Companies in the tesource-based view are seen as being basically heterogeneous. The resource-based view's perspective on the economic reality opens-up the "black-box" of the inside of the firm. In the resource-based view the reasons for performance differences between firms are reasons that lie within the firm itself, namely the resources or capabilities that every firm has. According to the resource-based view the economy is in a constant disequilibrium, which opens up opportunities for firms. Because of the different resources firms control, and thus the different resource endowments of the firms, performance differences occur. The resources or capabilities that firms posses are unique, scarce and difficult to imitate (Penrose 1959; Wernerfelt 1984; Barney 1991; Conner 1991; Peteraf 1993). These characteristics make it possible for firms possessing specific resources to yield teturns unattainable for firms lacking them, and thus supplying them a competitive edge.

In the classical resource-based view these resources are seen as "sticky", untradeable resources. Firms posses them because of some historical reason, they cannot sell them and they cannot buy them. This makes the classical resource-based theory "static" or "rigid". This rigidity is no problem in a stable economic environment with few changes. For modern day medium- and hightech sectors, which are characterised by swiftly changing competitive edges, this might actually be too rigid.

The dynamic capabilities view of the firms, in which not the exploitation of current resources is the pivotal focus point, but tather the change in the bundle of a firm's resources (Prahalad and Hamel 1990; Nelson 1991; Teece, Pisano et al. 1997), is better able to explain performance differentials in a turbulent economic environment. Since the firm"s environment is constantly changing, firms needs to change their bundle of resources in order to be able to meet the changing environmental requirements.

Differences in firm performances thus occur because of differences in a firm's capability to improve irs current core resources or to develop new ones (Teece, Pisano et al. 1997). The ability to improve on existing resources or to develop new ones is itself a core resource (Levitt and March 1988; Gilbert and Cordey-Hayes 1996; Teece, Pisano et al. 1997). For dynamic capabilities interorganisational learning is therefore a related and very important concept. Firms can only keep their capabilities dynamic when they are able to learn from other companies, or when they have the possibility to conduct a sufficient degree of R\&D themselves. As inter-organisational learning turns out to be of such critical importance to the dynamic capabilities of a firm, and thus to the firm's economic performance, it would be interesting to investigate it further, and to see what its characteristics are. 
Shedding light on the mechanism of how knowledge flows outside firm boundaries can aid us in a better understanding of how firms learn. Organisational learning is seen as an important soutce of competitive advantage, and is therefore regarded as an important factor explaining overall company success (Cohen and Levinthal 1989; Kogut and Zander 1992; Nonaka, Byosiere et al. 1994; Teece, Pisano et al. 1997; Edmondson, Bohmer et al. 2001). This realisation of the importance of knowledge for the growth of the economy, and consequently for the growth of the firm, is not new. Alteady in the 1930 soseph Schumpeter devoted extensive attention to knowledge and the importance of technological capability building in his work (Schumpeter 1934; Schumpeter 1939; Schumpeter 1942).

In this chapter we want to investigate the various main factors influencing the knowledge flowing from one firm to the other.

For knowledge to flow from one company to the other, and to be used by this other company, two factors are very important. First firms must be willing to use and adopt the external knowledge (Trott, Cordey-Hayes et al. 1995). Paralyzed by their core rigidities firms might be unable to recognize the importance of adopting new knowledge (Leonard-Batton 1995). Their current competencies might keep them trapped and blind for advantageous external innovations (Levitt and March 1988).

A second important factor is that there needs to be at least some degree of relatedness of the receiving firm's knowledge with the knowledge it gets from the other firm. Learning is only possible if the receiving firm can relate the new knowledge, to knowledge that is already part of its knowledge base (Bower and Hilgard 1981; Cohen and Levinthal 1990; Cohen 1991). Part of what companies learn from others is likely to be an echo of their own previous knowledge (Anderson 1848). Firms need to have some threshold level of absorptive capacity. Without sufficient absorptive capacity the new knowledge will be of no use to the receiving firm (Cohen and Levinthal 1990; Szulanski 1996). Cohen and Levinthal define absorptive capacity as the whole of the abilities of firms to use their prior related knowledge to value external information, assimilate it and use it for their own commercial ends (Cohen and Levinthal 1990). One way to see absorptive capacity in this view is to look at the specific knowledge base of the firm, where with specific knowledge base (SKB) we mean the total amount of knowledge available to the firm. Firms that possess more knowledge will have a higher absorptive capacity, since they posses more knowledge to which they can relate the new knowledge they are exposed to in the environment. This implies that firms with a larget specific knowledge base will be able to incorporate more outside knowledge, and thus learn more than firms with a smaller specific knowledge base. On the other hand, firms with a larger specific knowledge base have a higher chance of supplying other firms 
with knowlledge. The larger a firm's specific knowledge base the higher the chance that other firms can link it to their own knowledge and use it for their own benefit. The size of a firm's specific knowledge base would thus increases the knowledge flows to and from the company.

This leads us to our first set of hypotheses:

$\mathrm{H}_{\mathrm{ta}}$ : Firms that have a larger firm specific knowledge base have a higher degree of knowledge flows to other companies

\section{$\mathbf{H}_{1 \mathrm{~b}}$ : Firms that have a larger firm specific knowledge base receive a higher degree of knowledge flows from other companies}

The specific knowledge base of a firm can be interpreted as a static measure of absorptive capacity of a firm, it tells us something about the current state of the absorptive capacity of the firm. Besides the static measure of absorptive capacity we should also incorporate the dynamic factors influencing the absorptive capacity. One of the most important dynamic factors influencing the firm specific knowledge base is the amount of R\&D spending of the firm. Dierickx and Cool (1989: 1506) illustrate this using the "bathtub" metaphor. The level of water in the tub indicates the stock of water at any moment in time; it is a cumulative result of the flows of water into the tub. In our example this is the firm specific knowledge base, which represents the total stock of knowledge at a particular moment in time. The current $R \& D$ spending is than represented by the water flowing into the tub through the tap.

Firms invest in R\&D not only to pursue direct process and product innovations, but allso to maintain and develop their capabilities to assimilate and exploit external knowledge (Cohen and Levinthal 1989; Zahra and George 2002). The amount that firms spend on $R \& D$ influences the specific knowledge base of the firm, since $R \& D$ spending is seen as a good measure of firm performance in innovation (Verspagen 1996; Freeman and Soete 1997; Bloom and Reenen 2000). R\&D spending is thus often used by companies to learn, either by establishing R\&D units close to innovation centres, or by working together on lead markets or with lead customers (Niosi 1999). Via R\&D spending firms have a means of internal learning, which can aid them in learning form the external knowledge they encounter. The more firms spend on R\&D the more they can be expected to have a good understanding of the current state of knowledge in their field of research. On the one hand does this aid firms in assimilating the external knowledge within their own firm boundaries, on the other hand will firms with more $\mathbb{R} \& D$ efforts be more 
antractive partners for other firms that aim to gather valuable knowledge. Firms might invest in R\&D even though their efforts spill out into the economy as a whole for reasons of increasing their own absotptive capacity. This might ensure them a competitive edge (Cohen and Levinthal 1989). Other firms will. try to follow the R\&D intensive firm by trying to link up with their knowledge. R\&D spending thus increases the absorptive capacity of the firm, and increases the knowledge potential of the firm to other firms, which in turn will increase the knowledge flows to and from the company.

This brings us to our second set of hypotheses:

\section{$\mathrm{H}_{2 \mathrm{a}}$ : A firm's R\&D spending has a positive effect on the knowledge flows to other companies}

\section{$\mathrm{H}_{2 \mathrm{~b}}$ : A firm's $\mathrm{R} \& \mathrm{D}$ spending has a positive effect on the knowledge flows to the company}

Where $R \& D$ spending is a means of internal learning, firms can also learn directly from their external environment. This so-called external learning is often realised through means of strategic alliances (Kogut 1988; Hagedoorn and Schakenraad 1990; Hagedoorn 1993b; Mody 1993; Khanna, Gulati et al. 1998). Although internal learning via $R \& D$ efforts is very important for organisational learning, learning via alliances is becoming increasingly more important. The rapid technological changes of today's economic environment make it impossible, even for large multinational firms to develop everything themselves. The "bathtub" metaphor discussed before also illustrates this crucial point concerning knowledge bases; flows of knowledge can be adjusted immediately, while stocks of knowledge cannot. It takes time to accumulate the desired change in knowledge stock. Firms may not have this time to develop solutions to the problems they encounter internally (Dierickx and Cool 1989; lane and Lubatkin 1998). Therefore they need to work together and need to rely on the competences of other firms. Other forms of external knowledge appropriation, like M\&A's turn out to be less optimal in rapidly changing environments (Doz and Hamel 1998).

Since learning in alliances seems to be of such eminent importance we can expect to see more knowledge flows to and from firms with increasing numbers of alliances. So irrespective of the specific knowledge bases of the firms, and the familiarity of the allying firms with each others knowledge base, one can expect more knowledge flows to and from allying firms as opposed to non-allying firms (Hagedoorn and Schakenraad 1994). 
Our third set of hypotheses is therefore:

$\mathrm{H}_{3:}$ : Firms will have more knowledge flowing to other companies if they have more alliances

\section{$\mathrm{H}_{3 \mathrm{~b}}$ : Firms will have more knowledge flows to their company if they have more alliances}

\subsection{Data}

The data we use to test our hypotheses comes from various different sources (see also chapter 2). We started of with a selection of medium- to hightech companies who appeared on the Fortune 500 list of the 500 largest multinational companies worldwide in 1997. This resulted in a list of 171 multinationals spread over 12 sectors. For the firms in this list we searched the EPO (European Patent Office) database, to find the patents per firm. Because in EPO all the patent applicants are recorded by the unique applicant name, and not by "parent" company, this could mean that we missed many patents if we would only look for the "parent" company's name. Therefore, before looking at the EPO database we first constructed a "group" list per firm, based on the Dun \& Bradstreet Linkages database, in which we searched for every "subsidiary" company of the "parent" the accompanying EPO code and the associated patents. Having these "group" lists enabled us to find virtually all the patents of each multinational. The version of the Dun \& Bradstreet Linkages database we used is from late 1998, and thus represents the parent-subsidiary telationships at that point in time.

Fort the data on alliances we made use of the MERIT-CATT (Cooperative Agreements and Technology Indicators) database. The CATI database contains information on over 15,000 strategic alliances and holds information for over 3500 different parent companies. The CATI database contains only information related to technological cooperation, referring to those agreements wete a combined innovative activity or an exchange of technology is at least part of the agreement. Partnerships that regulate no more than the sharing of production facilities, the setting of standards, collusive behaviour in price setting and raising entry bartiers are omitted from the database.

The information on R\&D expenditures, revenues and number of employees was taken from the Worldscope database. 
The sectors the companies are involved in are the following: Aerospace (1); Chemicals (2); Computers, (3); Electronics (4); Semiconductors (5); Industrial and farm equipment (6); Metals (7); Motor vehicles (8); Petroleum refining (9); Pharmaceuticals (10); Scientific, photo, control equipment (11); and Telecommunications (12). All firms are multinational firms of which the headquarters are located worldwide with a main focus on the Triad USA, Europe and Japan.

\subsection{Methods}

The absorptive capacity of a firm, defined as the specific knowledge base of the firm, can be measured by the number of patents per firm. 'The more patents the higher the chance that the knowledge contained in these patents can be linked up with outside knowledge, and visa versa. We follow the reasoning of Jaffe and Trajtenberg (1998) that patents are a proxy for pieces of knowledge. A knowledge flow, following the same reasoning, can be thought of as a patent citation. Although patent citations might not always indicate a knowledge flow, they are still considered a useful indicator for the spread of knowledge (Jaffe and Trajtenberg 1998). Patent citations are the paper trail of the knowledge flows, for they are included by the patenting firm or the patenting officer to indicate where the knowledge contained in the patent

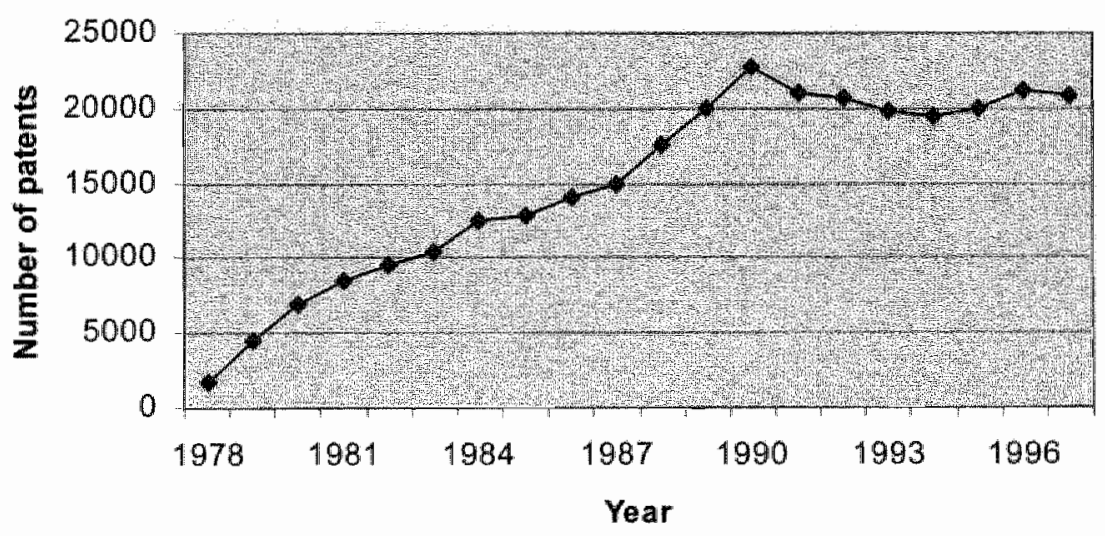

Figure 3.1: Number of patents per year 
comes from. Via patent citations we can thus more or less trace back the knowledge ancestors of each patent.

Besides the nomal problems associated with the use of patent statistics (Basberg 1987; Griliches 1990) (see also chapter 2), there are two more things we must keep in mind. One is that the number of patents applied for differs per year (see figure 3.1). This would mean that in the earliet years when there are less patents applied for, there is by definition, a smaller chance for patents to be cited. On the other hand patents that where taken out early, will have a longer citation listory, which gives them a higher chance on patent citations. We need to correct for both these problems.

The first problem can be solved by limiting the number of years to those years that have almost identical numbers of patents applied for. Looking at figure 3.1. we can see that this is the period 1989 till 1997. If we only use this period we can be pretry sure that the number of citations does not depend on the measuring years.

For the second problem to be solved we first need to look at the citationlag of the patents in our sample. For this reason we looked at the number of citations per patent for the first twenty years after the patent was applied for (see figure 3.2 ).

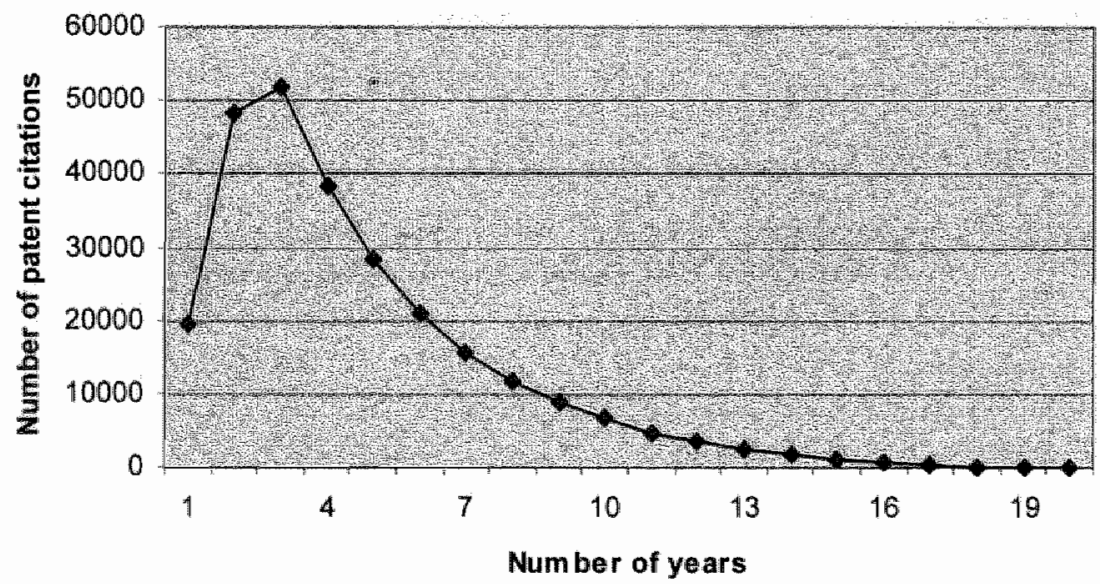

Figure 3.2: Citation-lag for the patents in the whole dataset.

Figure 3.2 shows that most citations occur between 4 and 5 years after the patent application. For our calculations we chose to cut off the number of citations to a patent four years after the application, which is also in line with earlier research (Hagedoorn and Cloodt 2003). This allows us to use five 
measuring years, since we measure the number of parent citations for the patents from 1989 till 1993 using a four-year mowing window. This means that for instance for the patents that were applied for in 1989 we measured all the patent citations until 1993. For the patents applied for in 1990, we measured the patent citations till 1994, etc. After this exercise we ended up with three statistics per firm, the number of patents applied for and the number of patent citations. Since there are two ways of looking at patent citations, the number of times the firm cites patents of other firms, and the number of times the patents of the firm are cited by other firms' patents, we included both values.

\section{Dependent yariable}

Our dependent variable is measured as the number of patent citations, both from the company to other companies (Citing) and from other companies to the company (Cited) for the time period 1989-1997, using a four-year moving window. Our dependent variable is thus a non-negative random integer-valued count variable. This means that normal ordinary least squares (OLS) regression will provide an incomplete description of the results. The necessary condition for an OLS regression of normal distribution is not met with count data, since the vatiable cannot be negative. We therefore need to turn to a discrete probability distribution.

\section{Independent variables}

As a first independent variable we used the total number of patents per: firm (Numpat). As discussed before we see patents as a proxy for pieces of knowledge (Jaffe and Trajtenberg 1998), and thus see a firm's absorptive capacity as the total number of patents per firm. According to our reasoning, a higher absorptive capacity would lead to more knowledge flows. We also regressed without this variable, with the logarithm of this variable (LnNumpat), to control for large size differences, and with the patent intensity of this firm (PatInt), defined as the number of patents per employee. This last variable does not consider the absolute size of the absorptive capacity of a firm, as do Numpat and LnNumpat, but instead looks at the influence of the relative size of a firm's absorptive capacity on knowledge flows.

As a further independent variable we used the $R \& D$ intensity of the firm (R\&DInt), defined as the amount spend on R\&D divided by the revenues of the firm. This variable is a good measure for the dynamic factors influencing the absorptive capacity. The more $R \& D$ intensive a firm is the more knowledge flows it can be expected to have. R\&D spending is generally considered a good indicator for firm performance in innovation (Verspagen 1996 ; Freeman and Soete 1997; Bloom and Reenen 2000), and is considered to have a positive influence on absorptive capacity (Cohen and Levinthal 1989). 
The last independent variable we used is the number of prior alliances of the firm (NumPA). We also used the logarithm of this variable in our regressions (LnNumPA) to control for large size difference between the companies on this variable. Since alliances are more and more used to learn from their alliance partners (Kogut 1988; Hagedoom and Schakenraad 1990; Hagedoorn 1993a), we can expect that firms with more alliances have a higher change of learning from their partners, and consequently will have more knowledge flows, especially in high-and medium-tech sectors.

\section{Contrat variables}

As a control variable for size we used the logarithm of the number of employees of the firm (SIZE). Dating back to Schumpeter, the argument was made that R\&D activity and patent activity of companies increases with their size (Schumpeter 1934; Scherer 1965; Kamien and Schwartz 1982; Scherer 1984; Mueller 1986; Cohen and Klepper 1996). Another reason for using this control variable is the clear evidence in the literature of a link between a company's size and its degree of alliance formation (Mytelka 1991; Duysters and Hagedoorn 1995; Hagedoorn 1995). Thus, though we are using only multinational firms in our dataset we want to control for size since there are considerable size differences, even between multinational companies, and we expect this to have an influence on our results.

We also included dummy variables for the 12 sectors in our dataset (Dum1-11). Our sample contains both high-tech and medium-tech sectors. High-tech sectors are almost by definition sectors with a high degree of patentintensity, and consequently will show a disproportionate share in industry wide patenting (Griliches 1998). There might however also be other industry influences, like the relative size of the sectors, which we need to control for.

To test our main hypotheses we use the following empirical specifications:

$$
\begin{aligned}
\text { Cited } & =f(\text { Numpat, LnNumpat, PatInt, R\&DInt, NumPA, LnNumPA, SIZE, } \\
\text { Dummy 1-11). } & \text {. }
\end{aligned}
$$

And:

$$
\begin{gathered}
\text { Citing }=f(\text { Numpat, LnNumpat, PatInt, R\&DInt, NumPA, LnNumPA, SIZE, } \\
\text { Dummy 1-11). }
\end{gathered}
$$




\subsection{Results}

In table 3.1 we report the descriptive statistics and correlations for our variables with Cited and Citing respectively, as our dependent vatiables. Table 3.2 shows the results for our dummy variables. It turns out that there are no setious correlations among the variables. The high correlations that we observe between the variables Numpat and LnNumpat and between NumPA and LnNumPA are not a problem, since these variables were never regressed simultaneously. We also looked at the Variance Inflation Factor for our regressions (not reported here). There turned out to be no serious multicollinearity among our variables, the VIF values were well below 4 (were 10 is the cut-off value).

Table 3.1 Means, Standard deviations and cortelations (N= 171) Dependent variable Cited and Citing

\begin{tabular}{|c|c|c|c|c|c|c|c|c|c|c|c|}
\hline Variables & Mean. & s.d. & I & 2 & 3 & 4 & 5 & 6 & 7 & 8 & 9 \\
\hline Cited & 136.30 & 221.35 & 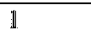 & & & & & & & & \\
\hline Citing: & 136.30 & 222.35 & & $\mathrm{~T}$ & & & & & & & \\
\hline Numpat & 609.63 & 885.48 & 0.896 & 0.922 & 1 & & & & & & \\
\hline LnNumpat & -29.97 & 185.34 & 0.621 & 0,617 & 0.681 & 1 & & & & & \\
\hline Pathnt & 2.206 & 2.566 & 0.454 & 0.453 & 0.503 & 0.515 & $\mathbb{1}$ & & & & \\
\hline R\&Dint & 0.026 & 0.019 & 0.511 & 0.511 & 0.566 & 0.586 & 0.472 & 1 & & & \\
\hline $\operatorname{NumP}^{\mathrm{P}} \mathrm{A}$ & 41.08 & 55.13 & 0.570 & 0.564 & 0.635 & 0.492 & 0.167 & 0.516 & 1 & & \\
\hline LnNumPA & -125.87 & 336.493 & 0.402 & 0.383 & 0.452 & 0.576 & 0.127 & 0.544 & 0.802 & 1 & \\
\hline LinumEmp & 11.014 & 0.943 & 0.416 & 0.428 & 0.447 & 0.401 & -0.140 & 0.285 & 0.498 & 0.510 & 1 \\
\hline Duml & 0.046 & 0.210 & -0.102 & -0.105 & -0.072 & 0.007 & .0 .072 & 0.104 & 0.084 & 0.126 & 0.081 \\
\hline Dum? & 0.094 & 0.290 & 0.092 & 0.109 & 0.215 & 0.213 & 0.289 & 0.192 & 0.104 & 0.133 & -0.070 \\
\hline Dimin3 & 0.047 & 0.210 & 0.323 & 0.309 & 0.256 & 0.122 & 0.137 & 0.107 & 0.200 & $\infty 0,0116$ & 0.064 \\
\hline Dum 4 & 0.150 & 0.350 & 0.408 & 0.387 & 0.300 & 0.272 & 0.015 & 0.149 & 0.209 & 0.164 & 0.294 \\
\hline Dums & 0.012 & 0.110 & 0.017 & 0.013 & 0.026 & -0.013 & 0.012 & 0.037 & 0.126 & 0.129 & -0.015 \\
\hline Dum6 & 0.047 & 0.210 & $-0.11 \overline{8}$ & -0.1110 & $-0,087$ & -0.009 & -0.076 & -0.101 & $.0,093$ & $=0.0119)$ & 0.043 \\
\hline Dum 7 & 0.076 & 0.270 & -0.136 & -0.139 & -0.122 & -0.025 & -0.036 & -0.249 & -0.159 & -0.067 & 0.129 \\
\hline Dump 8 & 0.150 & 0.350 & -0.150 & 0.131 & -0.117 & -0.014 & -0.181 & -0.047 & 0.025 & 0.043 & 0.199 \\
\hline Dumg & 0.180 & 0.390 & -0.212 & -0.219 & -0.224 & -0.234 & 0.004 & -0.425 & -0.222 & $\approx 0.302$ & $.0,426$ \\
\hline Dun 10 & 0.059 & 0.240 & 0.011 & -0.003 & 0.032 & 0.157 & 0.095 & 0.237 & 0.065 & 0.166 & .0 .0015 \\
\hline Dum II & 0.018 & 0.130 & 0.142 & 0.121 & 0.191 & 0.151 & 0.254 & 0.260 & -0.013 & 0.023 & 0.009 \\
\hline
\end{tabular}




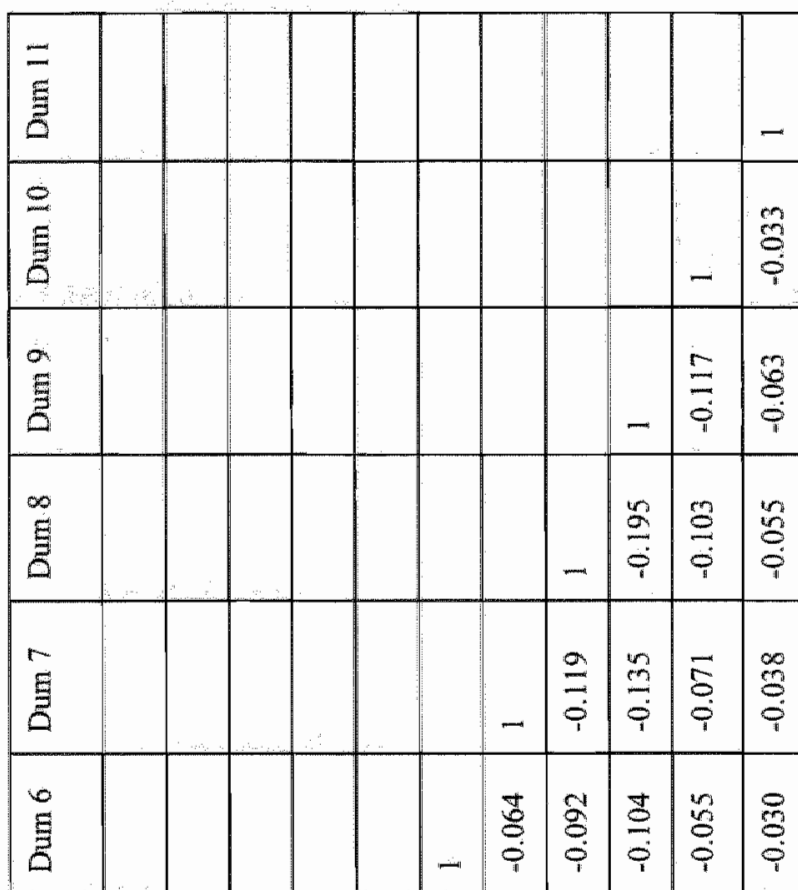

\begin{tabular}{|c|c|c|c|c|c|c|c|c|c|c|c|}
\hline 曋 & & & & & - & 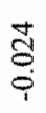 & $\overline{8}$ & $\begin{array}{l}\stackrel{9}{0} \\
\stackrel{0}{9}\end{array}$ & $\begin{array}{l}\overline{8} \\
8\end{array}$ & $\begin{array}{l}\text { 영 } \\
\text { i }\end{array}$ & $\frac{n}{8}$ \\
\hline$\stackrel{\vec{\Xi}}{\mathrm{g}}$ & & & & - & $\stackrel{8}{8}$ & $\frac{8}{8}$ & $\frac{9}{D}$ & $\vec{E}$ & $\frac{2}{9}$ & $\frac{8}{8}$ & $\stackrel{n}{8}$ \\
\hline$\stackrel{m}{B}$ & & & - & $\frac{\delta_{0}^{2}}{8}$ & $\stackrel{8}{8}$ & $\frac{a}{8}$ & $\begin{array}{l}\mathbb{8} \\
8\end{array}$ & $\frac{8}{8}$ & $\frac{d}{\dot{\theta}}$ & $\frac{6}{6}$ & $\stackrel{8}{9}$ \\
\hline 臬 & & - & 8 & $\frac{m}{m}$ & $\frac{1}{8}$ & $\frac{1}{8}$ & 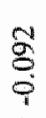 & $\frac{m}{9}$ & $\frac{\pi}{9}$ & $\begin{array}{l}8 \\
8 \\
8\end{array}$ & $\frac{9}{8}$ \\
\hline $\overrightarrow{5}$ & - & 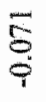 & $\frac{g}{8}$ & $\stackrel{8}{8}$ & $\stackrel{8}{8}$ & $\frac{9}{8}$ & 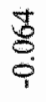 & $\frac{8}{8}$ & $\frac{8}{\phi}$ & $\begin{array}{l}2 \\
8 \\
8\end{array}$ & 8 \\
\hline Wh & $\frac{8}{8}$ & $\begin{array}{c}\stackrel{8}{9} \\
0\end{array}$ & $\underset{\mathrm{c}}{\mathrm{d}}$ & $\frac{8}{3}$ & $\frac{0}{0}$ & $\frac{0}{1}$ & 롱 & $\begin{array}{l}8 \\
0\end{array}$ & $\underset{8}{\stackrel{8}{8}}$ & $\stackrel{?}{\stackrel{7}{0}}$ & $\frac{8}{0}$ \\
\hline$\sum_{\mathbb{3}}^{\mathbb{3}}$ & $\stackrel{0}{0}$ & $\frac{\mathbb{8}}{8}$ & 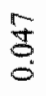 & $\frac{8}{0}$ & $\stackrel{0}{0}$ & $\stackrel{8}{8}$ & $\stackrel{0}{8}$ & $\frac{0}{0}$ & $\frac{\infty}{0}$ & $\begin{array}{l}0 \\
8 \\
8\end{array}$ & $\frac{\infty}{\delta}$ \\
\hline$\frac{\frac{3}{8}}{\frac{80}{5}}$ & $\bar{E}$ & $\frac{\mathrm{H}}{\mathrm{E}}$ & $\stackrel{?}{\Xi}$ & $\frac{\mathscr{E}}{E}$ & 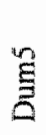 & $\stackrel{\mathscr{E}}{\mathscr{E}}$ & 氞 & $\stackrel{\stackrel{\infty}{\Xi}}{\Xi}$ & $\stackrel{g}{\vec{B}}$ & $\frac{\rho}{\tilde{\Xi}}$ & $\bar{\Xi}$ \\
\hline
\end{tabular}


Since our dependent variables consist of count data we cannot use standard OLS regression, but instead have to use Poisson or Negative Binomial Regression. The Poisson model is the statistical standard model for count data (Hausman, Hall et al. 1984; Cameron and Trivedi 1986). With the number of patent citations specified as Poisson we can regress them on the independent variables. The Poisson regression model has however one important restriction. The model is based on the assumption that the mean and the variance of the dependent variable are equal, given the independent variables. With many empirical count data distributions the variance however turns out to exceed the mean, leading to overdispersion. In case of overdispersion, the estimates of the Poisson regression model are still consistent yet inefficient. Inferences based on the estimated standard errors are thus no longer valid. The negative binomial regression model takes account of this overdispersion, and should be used in such circumstances (Hausman, Hall et al. 1984; Cameron and Trivedi 1986). The negative binomial regression model is a more general case of the Poisson regression model.

To test whether the Poisson regression model is applicable in this case we need to test the variance-mean equality, conditional on the explanatory variables. Standard tests to be used in such a case are the likelihood ratio test, the Wald test or t-test (Cameron and Trivedi 1990; Greene 2000). Cameron and Trivedi (1990) found that the optimal test involves simple least squares regressions, where they test the variance-mean equality, $\mathrm{H}_{0}: \operatorname{Var}\left(\mathrm{y}_{\mathrm{i}}\right)=\mu_{\mathrm{i}}$, against the alternative hypothesis $H_{A}: \operatorname{Var}\left(y_{i}\right)=\mu_{i}+\alpha g\left(\mu_{i}\right)$, where $g\left(\mu_{i}\right)=\mu_{i}$ or $\mathrm{g}\left(\mu_{\mathrm{i}}\right)=\mu_{\mathrm{i}}^{2}$, and $y_{\mathrm{i}}$ is the dependent variable. If $\alpha=0$, than the model reduces to a standard Poisson model, with the variance equal to the mean. The results of these tests for overdispersion are shown in tables 3.3 and 3.4 . 
Table 3.3 Tests for overdispersion in Poisson Model Dependent variable Cited.

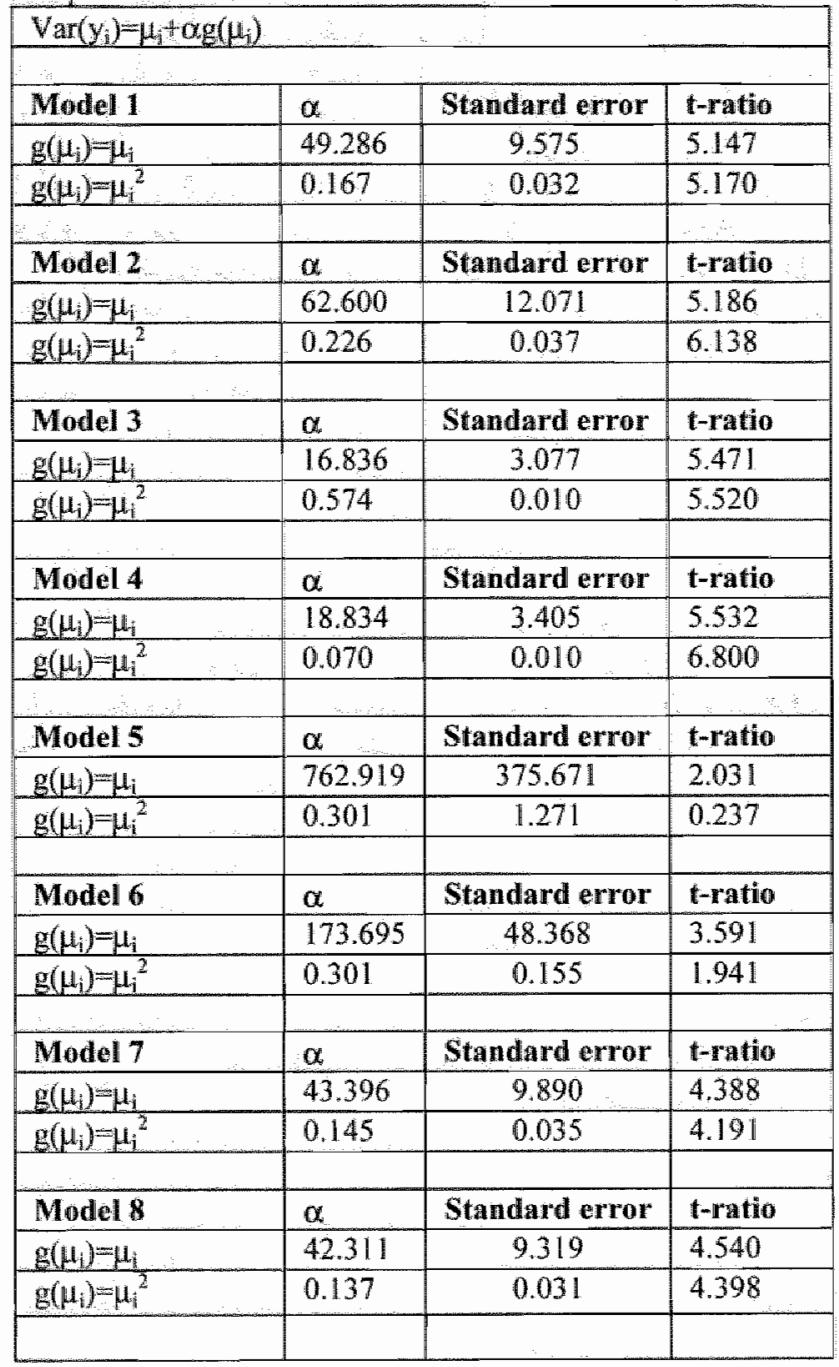


Table 3.4 Tests for overdispersion in Poisson Model Dependent variable Citing.

\begin{tabular}{|c|c|c|c|}
\hline $\operatorname{Var}\left(y_{i}\right)=\mu_{i}$ & & & \\
\hline Model 9 & $\alpha$ & Standard error & t-ratio \\
\hline$g\left(\mu_{j}\right)=\mu_{i}$ & 45.921 & 9.475 & 4.847 \\
\hline$g\left(\mu_{i}\right)=\mu_{i}^{2}$ & 0.162 & 0.031 & 5.232 \\
\hline Model 10 & $\alpha$ & Standard error & t-ratio \\
\hline$g\left(\mu_{i}\right)=\mu_{i}$ & 56.195 & 11.291 & 4.977 \\
\hline$g\left(\mu_{i}\right)=\mu_{i}^{2}$ & 0.220 & 0.033 & 6.721 \\
\hline Model 11 & $\alpha$ & Standard error & t-ratio \\
\hline$g\left(\mu_{i}\right)=\mu_{i}$ & 14.531 & 3.107 & 4.676 \\
\hline$g\left(\mu_{i}\right)=\mu_{i}^{2}$ & 0.053 & 0.010 & 5.060 \\
\hline Model 12 & $\alpha$ & Standard error & t-ratio \\
\hline$g\left(\mu_{i}\right)=\mu_{i}$ & 16.173 & 3.609 & 4.482 \\
\hline$g\left(\mu_{i}\right)=\mu_{i}^{2}$ & 0.060 & 0.011 & 5.316 \\
\hline Model 13 & $\alpha$ & Standard error & t-ratio \\
\hline$g\left(\mu_{i}\right)=\mu_{i}$ & 630.753 & 289.916 & 2.176 \\
\hline$g\left(\mu_{i}\right)=\mu_{i}^{2}$ & 0.314 & 0.970 & 0.324 \\
\hline Model 14 & $\alpha$ & Standard error & t-ratio \\
\hline$g\left(\mu_{i}\right)=\mu_{i}$ & 157.174 & 39.686 & 3.960 \\
\hline$g\left(\mu_{i}\right)=\mu_{i}^{2}$ & 0.300 & 0.127 & 2.356 \\
\hline Model 15 & $\alpha$ & Standard error & t-ratio \\
\hline$g\left(\mu_{i}\right)=\mu_{i}$ & 40.372 & 10.186 & 3.964 \\
\hline$g\left(\mu_{i}\right)=\mu_{i}^{2}$ & 0.142 & 0.035 & 4.009 \\
\hline Model 16 & $\alpha$ & Standard error & t-ratio \\
\hline$g\left(\mu_{i}\right)=\mu_{i}$ & 38.312 & 9.131 & 4.196 \\
\hline$g\left(\mu_{i}\right)=\mu_{i}^{2}$ & 0.129 & 0.030 & 4.239 \\
\hline
\end{tabular}


The tests show that the Poisson regression is not feasible in this case since there is clear evidence for overdisperion in the data. $\alpha$ is significantly different from zero in the first regression based test for every model we tested. For the models 5 and 13, $\alpha$ is not significant for the second tegression based test. Therefore we estimated the Negative Binomial model $\mathbb{I}$, following Hausman, Hall and Grilliches (1984) and Cameron and Trivedi (1986).

The tables 3.5 and 3.6 show the results of the Negative Binomial regressions, for both dependent variables and using different combinations of independent variables. In the models 1 till 8 we tested our hypotheses $1 \mathrm{a}, 2 \mathrm{a}$ and 3 a, using Cited as dependent variable. Models 9 till 16 were used to test our hypotheses $1 \mathrm{~b}, 2 \mathrm{~b}$ and $3 \mathrm{~b}$, whereby Citing is our dependent variable. 
Table 3.5 results for the negative binomial regressions, using different combinations of variables. $\mathrm{N}=171)$. Dependent variable Cited

\begin{tabular}{|c|c|c|c|c|c|c|c|c|}
\hline Variables & Model 1 & Model 2 & Model 3 & Modict 4 & Model 5 & Mothel 6 & Model 7 & Model \\
\hline Constant & $\begin{array}{l}0.838 \\
(2.372) \\
\end{array}$ & $\begin{array}{l}1.813^{3} \\
(1.960)\end{array}$ & $\begin{array}{l}0.716 \\
(0.903)\end{array}$ & $\begin{array}{l}0.322 \\
(1.020)\end{array}$ & $\begin{array}{l}10.713^{3 x} \\
0.318\end{array}$ & $\begin{array}{l}7.525^{*} \\
(0.306)\end{array}$ & $\begin{array}{l}-3.729 \% \text { 矃 } \\
(1.660)\end{array}$ & 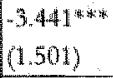 \\
\hline Numpat & $\begin{array}{l}5.071 \% \text { w } \\
(0.000)\end{array}$ & $\begin{array}{l}(4.910)^{*} \\
(0.000)\end{array}$ & & & & & & \\
\hline Loto Wampat & & & $\begin{array}{l}24.191 * * \\
(0.043)\end{array}$ & $\begin{array}{l}19.504 \text { w } \\
(0.055)\end{array}$ & & & & \\
\hline Pardnt & & & & & $\begin{array}{l}6.678 \\
(0.015) \\
\end{array}$ & $\begin{array}{l}6.676 \\
(0.015)\end{array}$ & $\left(\begin{array}{l}8.799 * \\
(0.013)\end{array}\right.$ & 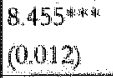 \\
\hline R\&Dint & $\left(\begin{array}{l}1.236 \\
(0.101)\end{array}\right.$ & $\begin{array}{l}1.425 \\
0.096)\end{array}$ & $\begin{array}{l}1.100 \\
(0.034)\end{array}$ & $\left(\begin{array}{l}0.513 \\
0.045)\end{array}\right.$ & $\begin{array}{l}2.420 \\
0.080)\end{array}$ & $\begin{array}{l}2.721 \\
(0.074)\end{array}$ & $\begin{array}{l}1.5 \% 6 \\
(0.078)\end{array}$ & $\begin{array}{l}1.746 \\
(0.073)\end{array}$ \\
\hline Nump $\mathrm{A}$ & $\begin{array}{l}0.336 \\
(0.003)\end{array}$ & & $\begin{array}{l}0.324 \\
(0.000)\end{array}$ & & $\begin{array}{l}3.243 \text { * } \\
0.002)\end{array}$ & & $\begin{array}{l}1.134 \\
(0.002)\end{array}$ & \\
\hline LunNumPA & & $\begin{array}{l}2.675 \\
(0.092)\end{array}$ & & $\begin{array}{l}1.444 \\
(0.052)\end{array}$ & & $\begin{array}{l}5.266 * 2 \\
(0.068)\end{array}$ & & $\begin{array}{l}2.562 \% \\
0.073)\end{array}$ \\
\hline Lon NumEmp & $\begin{array}{l}0.719 \\
(0.201)\end{array}$ & $\begin{array}{l}\infty 0.375 \\
(0.169) \\
\end{array}$ & $\begin{array}{l}-2.059 \text { * } \\
(0.082)\end{array}$ & $\begin{array}{l}-1.128 \\
(0.099 \\
\end{array}$ & & & $\begin{array}{l}5.849 \\
(0.14 .5) \\
\end{array}$ & $\begin{array}{l}5.3044 \text { as } \\
0.135 \\
\end{array}$ \\
\hline Duml & $\begin{array}{l}-1.967 \\
(0.626)\end{array}$ & $\begin{array}{l}-2.820^{*} \\
(0.514)\end{array}$ & $\begin{array}{l}-3.772 \text { w**** } \\
(0.322)\end{array}$ & 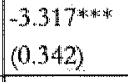 & $\begin{array}{l}-3.443^{* * * 3} \\
(0.511)\end{array}$ & $\begin{array}{l}-3.908 * * * \\
(0.444)\end{array}$ & $\begin{array}{l}-3.141 . \\
(0.460)\end{array}$ & $\begin{array}{l}-3.449 \\
(0.414)\end{array}$ \\
\hline Dum2 & $\begin{array}{l}0.971 \\
(0.619)\end{array}$ & $\begin{array}{l}-0.848 \\
(0.579) \\
\end{array}$ & $\begin{array}{l}-4.310^{k} \\
(0.225)\end{array}$ & $\begin{array}{l}-4.141^{* * * *} \\
(0.230)\end{array}$ & $\begin{array}{l}-2.617^{*} \\
(0.532)\end{array}$ & $\begin{array}{l}2.353^{4: 2} \\
(0.502)\end{array}$ & $\begin{array}{r}-1.239 \\
(0.497)\end{array}$ & $\begin{array}{l}-1.831 \\
(0.434)\end{array}$ \\
\hline Dum3 3 & $\begin{array}{l}0.114 \\
(0.629) \\
\end{array}$ & $\begin{array}{l}0.124 \\
(0.500)\end{array}$ & $\begin{array}{l}-1.811 * \\
(0.224)\end{array}$ & $\begin{array}{l}-0.644 \\
(0.268) \\
\end{array}$ & $\begin{array}{l}-0.807 \\
(0.426)\end{array}$ & $\begin{array}{l}0.074 \\
(0.387)\end{array}$ & $\begin{array}{l}-0.179 \\
(0.466) \\
\end{array}$ & $\begin{array}{l}0.111 \\
(0.408)\end{array}$ \\
\hline Dum4 & $\begin{array}{l}0.156 \\
(0.519)\end{array}$ & $\begin{array}{l}0.351 \\
(0.427)\end{array}$ & $\begin{array}{l}-1.747 \\
0.169)\end{array}$ & $\begin{array}{l}1.444 \\
(0.184)\end{array}$ & $\begin{array}{l}0.574 \\
(0.373)\end{array}$ & $\begin{array}{l}1.250 \\
(0.331)\end{array}$ & $\begin{array}{l}0.255 \\
(0.375)\end{array}$ & $\begin{array}{l}0.875 \\
(0.335)\end{array}$ \\
\hline Dum 5 & $\begin{array}{l}40.339 \\
(0.870) \\
\end{array}$ & $\begin{array}{l}-1.093 \\
(0.669)\end{array}$ & $\begin{array}{l}-13.465 \\
(0.198)\end{array}$ & $\begin{array}{r}-0.566 \\
(0.660)\end{array}$ & $\begin{array}{l}-2.158 \\
(0.878) \\
\end{array}$ & $\begin{array}{l}-2.020^{* * * *} \\
(0.858)\end{array}$ & $\begin{array}{l}1.552 \\
0.655) \\
\end{array}$ & $\begin{array}{l}-1.601 \\
0.598)\end{array}$ \\
\hline Dumb & $\begin{array}{l}-2.180^{* *} \\
(0.647)\end{array}$ & $\begin{array}{l}0.922 \\
(0.861) \\
\end{array}$ & $\begin{array}{l}-6.883^{*} * *+2 \\
(0.277)\end{array}$ & $\begin{array}{l}-3.752^{\text {**..kt }} \\
(0.461)\end{array}$ & $\begin{array}{l}-3.325 \text { : } \\
(0.445)\end{array}$ & $\begin{array}{l}-0.966 \\
(0.731)\end{array}$ & $\begin{array}{l}-2.621^{\text {s.t. }} \\
(0.498)\end{array}$ & $\begin{array}{l}1.875 * \\
0.613)\end{array}$ \\
\hline Dum? & $\begin{array}{l}1.080 \\
(0.532)\end{array}$ & $\begin{array}{l}-1.227 \\
(0.473) \\
\end{array}$ & $\begin{array}{l}-5.193^{3} \text { ). } \\
(0.200)\end{array}$ & 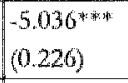 & $\begin{array}{l}2.522^{w} \\
(0.389)\end{array}$ & $\begin{array}{l}-1.756^{*} \\
(0.375)\end{array}$ & $\begin{array}{l}1.163 \\
(0.394) \\
\end{array}$ & $\begin{array}{l}-1.414 \\
(0.373) \\
\end{array}$ \\
\hline Dums & $\begin{array}{l}-1.239 \\
(0.512)\end{array}$ & $\begin{array}{l}-1.576 \\
(0.378)\end{array}$ & $\begin{array}{l}-4.680^{\text {nas }} \\
(0.182)\end{array}$ & 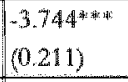 & $\begin{array}{l}-1.486 \\
(0.394)\end{array}$ & $\begin{array}{l}-1.401 \\
(0.318)\end{array}$ & $\begin{array}{l}1.708^{\text {*t }} \\
(0.353) \\
\end{array}$ & $\begin{array}{l}2.053^{*} \\
0.300 \\
\end{array}$ \\
\hline Dum9 & $\begin{array}{l}0.444 \\
(0.5434) \\
\end{array}$ & $\begin{array}{l}-0.475 \\
(0.433)\end{array}$ & $\begin{array}{l}-4.235^{\text {*a* * }} \\
(0.213) \\
\end{array}$ & $\begin{array}{l}-3.381 \text { * } \\
(0.238)\end{array}$ & $\begin{array}{l}-1.838 * \\
(0.373)\end{array}$ & $\begin{array}{l}-1.359 \\
(0.315)\end{array}$ & $\begin{array}{l}0.31 .4 \\
(0.391 y \\
\end{array}$ & $\begin{array}{l}-0.494 \\
0.320 \\
\end{array}$ \\
\hline Dun 10 & $\begin{array}{l}-0.324 \\
(0.632)\end{array}$ & $\begin{array}{l}0.254 \\
(0.765) \\
\end{array}$ & $\begin{array}{l}-3.037 \\
0.230)\end{array}$ & $\begin{array}{l}-2.668 \text { **1.4 } \\
(0.250)\end{array}$ & $\begin{array}{l}0.975 \\
(0.630)\end{array}$ & $\begin{array}{l}-1.996^{* * \text { *1 }} \\
\left(0.477^{1}\right)\end{array}$ & $\begin{array}{l}0.774 \\
0.473 \\
\end{array}$ & $\begin{array}{l}-0.811 \\
(0.471) \\
\end{array}$ \\
\hline Dum 11 & $\begin{array}{l}0.074 \\
(0.829)\end{array}$ & $\begin{array}{l}0.142 \\
(0.675)\end{array}$ & $\begin{array}{l}2.007 * 3 \\
(0.346)\end{array}$ & $\begin{array}{l}-2.108^{\text {*it }} \\
(0.341)\end{array}$ & $\begin{array}{l}-0.987 \\
(0.955)\end{array}$ & $\begin{array}{l}-1.368 \\
(1.141)\end{array}$ & $\begin{array}{l}-0.775 \\
(1.134)\end{array}$ & $\begin{array}{l}.0 .840 \\
0.969)\end{array}$ \\
\hline
\end{tabular}

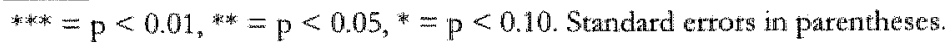




\section{CHAPTER III}

Table 3.6 results for the negative binomial tegressions, using different combinations of variables. $(\mathrm{N}=171)$. Dependent variable Cicing

\begin{tabular}{|c|c|c|c|c|c|c|c|c|}
\hline Variables & Model 9 & Model 10 & Model 11 & Modul 12 & Model 13 & Model 14 & Podxel 15 & Nodel 16 \\
\hline Combtains & $\begin{array}{l}0.333 \\
(1.835)\end{array}$ & $\begin{array}{l}1.974 \\
1587\end{array}$ & $\begin{array}{l}20.537 \\
100911\end{array}$ & $\begin{array}{l}-1.224 \\
(0.774)\end{array}$ & $\begin{array}{l}11.244 \\
0.302)\end{array}$ & $\begin{array}{l}8.069 \text { Ping } \\
0.319 \\
\end{array}$ & $\begin{array}{l}-3.737 \\
(1.1769 \\
\end{array}$ & $\begin{array}{l}3,476 \% \\
(1,298)\end{array}$ \\
\hline Whampat & $\begin{array}{l}4.892 \\
0.000)\end{array}$ & $\begin{array}{l}7.498 \text { क } \\
0.000\end{array}$ & & & & & & \\
\hline L nnvumagat: & & & $\begin{array}{l}19.092 \\
(0.049)\end{array}$ & $\begin{array}{l}20.277^{14 *} \\
(0.046)\end{array}$ & & & & \\
\hline Patitrut & & & & & $\begin{array}{l}7.900^{\text {sk }} \\
(0.013)\end{array}$ & $\begin{array}{l}7.119 \text { was } \\
(0.014)\end{array}$ & $\begin{array}{l}10.715^{\text {*a* }} \\
00.010\end{array}$ & $\begin{array}{l}8.624^{2 *} \\
(0.012)\end{array}$ \\
\hline Qx Dint & $\begin{array}{l}2.310^{2} \\
(0.088)\end{array}$ & $\begin{array}{l}2.237^{*} \\
(0.051)\end{array}$ & $\begin{array}{l}0.190 \\
(0.023)\end{array}$ & $\begin{array}{l}0.139 \\
(0.034) \\
\end{array}$ & $\begin{array}{l}2.760^{*} * \\
(0.066)\end{array}$ & $\begin{array}{l}1.802 \\
10.060\end{array}$ & $\begin{array}{l}1.218 \\
(0.050)\end{array}$ & $\begin{array}{l}1.845^{*} \\
(0.067) \\
\end{array}$ \\
\hline Wum ${ }^{A}$ & $\begin{array}{l}0.233 \\
(0.003)\end{array}$ & & $\begin{array}{l}-0.361 \\
(0.001)\end{array}$ & & $\begin{array}{l}3.988 \text {. } \\
(0.002)\end{array}$ & & $\begin{array}{l}1.684 \\
(0.002)\end{array}$ & \\
\hline LITHLEMPA & & $\begin{array}{l}2.440^{\text {* }} \\
(0.071)\end{array}$ & & $\begin{array}{l}-0.755 \\
(0.043) \\
\end{array}$ & & $\begin{array}{l}5.268 \\
(0.063) \\
\end{array}$ & & $\begin{array}{l}2.052^{\text {nit }} \\
(0.073)\end{array}$ \\
\hline LaNund anp & $\begin{array}{l}1.699 \% \\
(0.159) \\
\end{array}$ & $\begin{array}{l}0.046 \\
(0.141)\end{array}$ & $\begin{array}{l}0.355 \\
(0.086)\end{array}$ & $\begin{array}{l}0.979 \\
(0.070)\end{array}$ & & & $\begin{array}{l}6.658 \\
0.105)\end{array}$ & $\begin{array}{l}5.630^{2} \\
(0.121)\end{array}$ \\
\hline Diame & $\begin{array}{l}-2.464^{\text {s.3 }} \\
(0.607)\end{array}$ & $\begin{array}{l}-2.056^{\text {m }} \\
(0.5860\end{array}$ & $\begin{array}{l}-5.222^{*} \text { *小 } \\
(0.308\end{array}$ & $\begin{array}{l}-5.908 \% \\
(0.268)\end{array}$ & $\begin{array}{l}-3.833^{14 \%} \\
(0.461)\end{array}$ & $\begin{array}{l}-2.522^{\text {a }} \\
(0.520)\end{array}$ & $\begin{array}{l}-2633 \\
(0.432)\end{array}$ & $\begin{array}{l}-5.088 \text { (0.30) } \\
(0.370)\end{array}$ \\
\hline Duand 2 & $\begin{array}{l}\times 0.470 \\
(0.621)\end{array}$ & 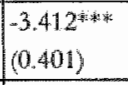 & $\begin{array}{l}-3.896 \\
0.284)\end{array}$ & $\begin{array}{l}-4.232 \text { क*⿻一未丷 } \\
(0.233) \\
\end{array}$ & $\begin{array}{l}-3.54496 * 1 \\
(0.443)\end{array}$ & $\begin{array}{l}2.533 \\
(0.434)\end{array}$ & $\begin{array}{l}-1.452 \\
0.378 \\
\end{array}$ & $\begin{array}{l}3.437^{* 3.3} \\
(0.358) \\
\end{array}$ \\
\hline Dums & $\begin{array}{l}0.049 \\
(0.559)\end{array}$ & $\begin{array}{l}0.042 \\
(0.515)\end{array}$ & $\begin{array}{l}-0.896 \\
(0.317 \\
\end{array}$ & $\begin{array}{l}-1.343 \\
(0.262) \\
\end{array}$ & $\begin{array}{l}-1.021 \\
(0.423)\end{array}$ & $\begin{array}{l}0.168 \\
(0.411) \\
\end{array}$ & $\begin{array}{l}-0.430 \\
(0.339)\end{array}$ & $\begin{array}{r}-0.120 \\
(0.474) \\
\end{array}$ \\
\hline Down & $\begin{array}{l}0.319 \\
(0.439)\end{array}$ & $\begin{array}{l}0.694 \\
(0.309) \\
\end{array}$ & $\begin{array}{l}-2.239 \\
(0.217)\end{array}$ & $\begin{array}{l}2.290^{\circ} \\
(0.189)\end{array}$ & $\begin{array}{l}0.806 \\
0.358) \\
\end{array}$ & $\begin{array}{l}1.864 \\
(0.334)\end{array}$ & $\begin{array}{l}1.480 \\
(0.248)\end{array}$ & $\begin{array}{l}1.014 \\
(0.291) \\
\end{array}$ \\
\hline Dumb & $\begin{array}{r}-0.182 \\
(0.935)\end{array}$ & $\begin{array}{l}-0.748 \\
(0.668) \\
\end{array}$ & $\begin{array}{l}-3.692 \\
0.305)\end{array}$ & $\begin{array}{l}-0.323 \\
(0.669) \\
\end{array}$ & $\begin{array}{l}-1.534 \\
(1.154) \\
\end{array}$ & $\begin{array}{l}-2.889 \text { *⿻一𠃋十: } \\
(0.613)\end{array}$ & $\begin{array}{l}-2937^{\text {(⿻) }} \\
(0.399) \\
\end{array}$ & $\begin{array}{l}-2.148^{*} \\
(0.558) \\
\end{array}$ \\
\hline Dhan6: & $\begin{array}{l}-2.398^{*} \\
(0.490)\end{array}$ & $\begin{array}{r}-1.460 \\
(0.540) \\
\end{array}$ & $\begin{array}{l}6.556 \\
0.284\end{array}$ & $\begin{array}{l}-5.619 \% \\
(0.287)\end{array}$ & $\begin{array}{l}-2.686^{* * *} \\
0.380 \\
\end{array}$ & $\begin{array}{l}1.015 \\
(0.500)\end{array}$ & $\begin{array}{l}-3.303^{\text {ind }} \\
(0.302) \\
\end{array}$ & $\begin{array}{l}-1.562 \\
0.485) \\
\end{array}$ \\
\hline 1Dham & $\begin{array}{l}-1.078 \\
(0.529) \\
\end{array}$ & $\begin{array}{l}-1.947 * \\
(0.461)\end{array}$ & 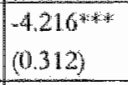 & 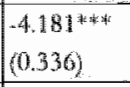 & $\begin{array}{l}-2.335^{\text {kn }} \\
(0.423)\end{array}$ & $\begin{array}{l}2.047 \\
0.412\end{array}$ & $\begin{array}{l}-2766 \\
(0.283 y\end{array}$ & $\begin{array}{l}-1.942^{4} \\
(0.376)\end{array}$ \\
\hline Dumb & $\begin{array}{l}1.326 \\
(0.438)\end{array}$ & $\begin{array}{r}-1.424 \\
0.3645 \\
\end{array}$ & $\begin{array}{l}-4.543^{2} \text { 米: } \\
(0.214) \\
\end{array}$ & 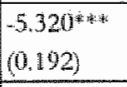 & $\begin{array}{l}-1.224 \\
(0.353) \\
\end{array}$ & $\begin{array}{l}-0.620 \\
(0.320 \\
\end{array}$ & $\left(\begin{array}{l}2358^{4} \\
0.225\end{array}\right.$ & $\begin{array}{l}2.121^{*: *} \\
(0.262)\end{array}$ \\
\hline Dum 9 & $\begin{array}{l}-0.625 \\
0.434 \\
\end{array}$ & $\begin{array}{l}-1.242 \\
(0.391)\end{array}$ & $\begin{array}{l}4.818^{\text {th }} \\
0.250\end{array}$ & $\begin{array}{l}-5.359^{*} * \\
(0.212)\end{array}$ & $\begin{array}{l}2.413 \\
0.345\end{array}$ & $\begin{array}{l}-2.009 \\
(0.321)\end{array}$ & $\begin{array}{l}2.345 \\
(0.237)\end{array}$ & $\begin{array}{l}-1.612 \\
(0.283) \\
\end{array}$ \\
\hline$D \min 10$ & $\begin{array}{l}0.514 \\
0.733)\end{array}$ & $\begin{array}{l}-3.9355^{4} \\
(0.386)\end{array}$ & 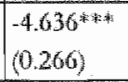 & $\begin{array}{l}2.491^{*} \\
(0.316)\end{array}$ & $\begin{array}{l}-0.514 \\
(0.644)\end{array}$ & $\begin{array}{l}1.153 \\
(0.573) \\
\end{array}$ & $\begin{array}{l}0.049 \\
(0.505)\end{array}$ & $\begin{array}{l}-1.916^{*} \\
(0.376)\end{array}$ \\
\hline Dum 1 & $\begin{array}{l}0.228 \\
0.962)\end{array}$ & $\begin{array}{l}0.267 \\
0.812)\end{array}$ & $\begin{array}{l}-7.658 \text {. } \\
(0.288)\end{array}$ & $\begin{array}{l}-4.065 * * \\
(0.312)\end{array}$ & $\begin{array}{l}-1.694^{+k} \\
(0.949)\end{array}$ & $\begin{array}{l}-3.168^{\text {事的和 }} \\
(0.558)\end{array}$ & $\left(\begin{array}{l}1.219 \\
(0.844\end{array}\right.$ & $\begin{array}{l}-4.64)^{*} \\
(0.442)\end{array}$ \\
\hline
\end{tabular}

* $=p<0.01^{*} * p^{*}=0.05_{*}^{*}=p<0.10$. Standatd ertors in parentheses. 
We will first look at liypotheses $1 \mathrm{a}$ and $1 \mathrm{~b}$, where we argued that knowledge flows to and from the company will be positively related to the level of the specific knowledge base of the firm. We see that for all the models we tested our independent variables Numpat, LnNumpat and Patlnt are positive and highly significant. This provides strong support for both hypothesis la and hypothesis $1 \mathrm{~b}$. The size of the specific knowledge base of a company and thus the absorptive capacity of a company cleatly has a positive influence on the knowledge flowing to and from the company.

We now turn to hypotheses $2 \mathrm{a}$ and $2 \mathrm{~b}$ where we asserted that a firms R\&D spending is positively related to the knowledge flows to and from the company. We find strong evidence for both hypotheses, although the evidence is somewhat stronger for hypothesis $2 \mathrm{a}$ than for $2 \mathrm{~b}$. In two models we find a negative beta for R\&DInt, but both coefficients are non-significant. For all the other models we find a positive coefficient, which is most of the time significant. This all points to cleat evidence for a positive relationship between R\&D spending and knowledge flows over firm botders, and supplies strong evidence for both hypothesis $2 \mathrm{a}$ and hypothesis $2 \mathrm{~b}$.

In our last two hypotheses we are investigating the influence of prior alliances on the knowledge flows to and from the company. We postulated that knowledge flows to and from the company increase, if these companies were engaged in more alliances. Our results are somewhat less strong than for our other hypotheses, but still most coefficients are positive and significant. Only for three models we do find a negative result, but none of them is significant. As we expected, the amount of prior alliances does have a positive influence on the knowledge flowing to and from the company. Both our hypotheses 3a and $3 \mathrm{~b}$ are thus supported by our results.

For our control variable for firm size (SIZE) we find clear positive evidence between this vatiable and the knowledge flows from other companies to the company. Apparently larger firms tend to cite other firms mote than do smaller firms. This is not surprising considering that larger firms will be involved in a larger spectrum of research. The evidence of SIZE and the knowledge flowing from the focal company to other companies is less conclusive. For three models we do find a negative result, one of which is significant. "The results are thus more or less inconclusive although there is a slight positive result, indicating that larger firms are cited mote. However, given the results we are unable to make a conclusive statement here. An explanation could be that some small firms are very knowledge intensive, where on the other hand large firms most often have a larger pool of knowledge. Both these results could offset one another more or less leading to the results we find. More research will however be needed here.

Considering the results for our dummy variables, we do not see a clear line, although most coefficients are negative. None of the sectors stands out 
clearly, and conclusions are difficult to draw from these results. More regtessions were carried out using different combinations of variables or leaving out some of the variables (not reported), but this did not have an influence on our tesults.

\subsection{Discussion and Conclusions}

In this chapter we empirically tested the factors influencing knowledge flows between multinational companies. Using the dynamic capabilities view of the firm we hypothesised that a larger firm specific knowledge base would increase the knowledge flows to and from the company. Our empirical results clearly show that there is indeed a strong positive relationship between a firm's absorptive capacity and the amount of knowledge flowing in and out of the company. On the one hand does a larger absorptive capacity allow firms to hook on to outside knowledge more easily, thus increasing the knowledge flows to the company. On the orher hand does this same larger absorptive capacity supply other firms with more possibilities to hook on to the firm's specific knowledge, thus leading to increased knowledge flows out of the company.

In our second set of hypotheses we postulated that a firm's $R \& D$ spending would have a positive effect on the knowledge flows to and form the company. Also here our empirical results provide clear support for our hypotheses. Firms that spend more on $R \& D$ will be better able to absorb and valuate the knowledge from the environment, which leads to more knowledge flows to the company. For other firms it would also be much more interesting to tap into the specific knowledge base of an $R \& D$ active firm than of one who relies on relatively old knowledge. New knowledge can be expected to provide firms with a competitive edge. New knowledge can generally be found in R\&D active firms, thus leading to more knowledge flows from R\&D intensive firms to other firms.

In our final set of hypotheses we argue that firms with a higher number of alliances will show more knowledge flows. Our empirical results again provide conclusive evidence for the anticipated relationship. More and more, alliances are established especially with the explicit purpose of knowledge exchange (Mowery, Oxley et al. 1996; Lane and Lubatkin 1998). Overall the positive relationship between knowledge flows and the establishment of alliances between these companies is in line with contemporary research (Hagedoorn 2002). These knowledge flows will of course be bi-directional.

Overall we can conclude that knowledge flows, which are so important for company progress, are clearly influenced by the three factors that we studied in this chapter i.e, absorptive capacity, R\&D intensity and the number of prior alliances. A firm's stock of knowledge, its relative efforts in R\&D and 
the amount of cooperation"s with other companies is shown to have a positive influence on the knowledge flowing in and out of the company. Since knowledge flows are so important for organisational learning and thus for company success, it is of eminent importance to understand which factors influence these knowledge flows in which way. This chapter aids to enlance our current understanding of this specific relationship, and shows that learning organisations in high- and medium-tech sectors need to work on their absorptive capacity by investing in $\mathrm{R} \& \mathrm{D}$ and by forming alliances. More research is needed in this field to fine-tune the influence of the individual factors and to search for possible other factors. 


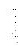




\section{CHAPTER 4 \\ Learning in Strategic Technology Alliances}

This chapter is hased on the article "Learning in Strategic Technology Alliances", by Wilfred Scliperimakers and Geert Duysters:

\subsection{Introduction}

The resources or capabilities of a firm are often seen as an important contribuant to overall company success and competitiveness (Penrose 1959; Prahalad and Hamel 1990; Barney 1991; Teece, Pisano et al. 1997). In the "traditional" resource-based view of the firm, firms are viewed as a collection of distinctive and difficult to imitate, scarce resources or capabilities (Penrose 1959; Wernerfelt 1984). The deployment of these valuable, rare and idiosyncratic tesources is expected to yield a distinct return or rent for the firm possessing them. Firm resources are thus, necessarily, seen as being heterogeneous across firms. This "traditional" resource-based perspective sees a firm's bundle of resources as static and more or less fixed over time. From this perspective firms are only able to acquire new resources through mergers or acquisitions.

Whereas the "traditional" resource-based view is mainly concerned with static competences, the dynamic capabilities view of the firm, concentrates on dynamic factors (innovation, organisational learning, etc.). Not the deployment of existing resources is at the focal point of this theory, but rather the change in a firm's resources (Teece, Pisano et al. 1997). This change in resources is necessary for firms in order to be able to respond effectively to changing environmental conditions. One of the dynamic capabilities view's main focus points is the acquisition of new capabilities through organisational learning.

Over time, consensus grew that organisational learning can be considered as the most important vehicle for competence development (Drejer 2000). Following Cohen and Levinthal (1989), we see that two characteristics of the innovation process are very important: the creation of new knowledge within the firm itself, and the incorporation of existing external knowledge. On this junction of internal and extemal knowledge accumulation one often finds strategic technology alliances. Here the internal knowledge inherent to the company is combined with knowledge external to the company (Hagedoorn and Duysters 2002). Both the resource-based view and the dynamic capabilities view help us to explain organisational learning within strategic alliances. However, they are unable to explain some of the key issues related to learning 
alliances. Therefore we turn to a third stream of literature, the knowledge-based view of the firm. In the knowledge-based view of the firm (Grant and BadenFuller 1995; Foss 1996) knowledge is considered the pivotal resource of a firm. Knowledge can consist of codified knowledge contained in the patents or copyrights of a firm, but can also be incorporated in the tacit everyday routine operations carried out by workers. In line with the dynamic capability perspective this view also concentrates on the dynamics of the firm resources, rather than on its static posture. An important point of the knowledge-based view is that it provides a new explanation for the observed trend towards collaborative agreements between firms. It has been shown that firms collaborate, among other things, to get access to the knowledge of other firms (Hagedoorn 1993b). According to a recent Accenture Study (1999) learning was cited as a critical goal in over $40 \%$ of all alliances under study. This percentage was expected to exceed 50 percent in 2003. As a result the use of collaborations is seen as an important vehicle for organisational learning and knowledge acquisition, and thus for the forming of new competencies.

Given the increasing importance of external knowledge appropriation by means of strategic alliances, it is of eminent importance to understand the particular nature of strategic technology partnering and to take a closer look at the impact of firm collaborations on organisational learning. In the rest of this chapter we will therefore explore the influence of strategic technology alliances on organisational learning.

\subsection{Theory and hypotheses}

Whereas the "traditional" resource-based view concentrates primarily on the efficient use of internal comperencies, the dynamic capabilities view of the firm argues that it is of vital importance to exploit external sources of capabilities. The knowledge-based view incorporates both perspectives and deals with the role of knowledge acquisition and integration within an organisational learning setting.

Firm specific capabilities are often difficult to create or imitate by other companies. Some capabilities are protected by patent law, while others are so idiosyncratic that taken out of their context they are hard to understand. Time constraints can hinder firms to create or imitate capabilities fast enough to be able to exploit them. Even firms possessing firm specific capabilities may not be able to use them effectively in other situations or other markets (Mowery 1983). Also the market for capabilities is not perfect, making it difficult to obtain the resources externally (Teece, Pisano et al. 1997). The external acquisition of knowledge via a merger or acquisition is also complicated. Whereas M\&A's can provide scale economies to organisations they hamper 
Alexibility, efficient knowledge transfer, and speed, the capabilities needed most in today's economy. As in the case of strategic alliances, recent studies have shown that, in spite of the unprecedented increase in the number of M\&A $\mathrm{s}_{\text {, }}$ their overall contribution to firms" performance is very poor: Their poor track record is primarily due to the massive integration challenges that arise after the acquisition in combination with the high acquisition premiums. Companies often get more than they want and need to sell parts of the acquired firm again. It might also be very difficult to use the acquired competences in the acquiring firm (Doz and Hamel 1998; Capron 1999). The most significant problem however, seems to be that the high costs associated with the mexger or acquisition and the correspondingly high exit costs diminish the Alexibility of firms to quicklly adapt to turbulent changes in the economy. Strategic alliances suffer less from these problems, for they allow greater flexibility and they are less involving than for instance an acquisition. A firm can also be involved in several alliances at a time, something that is more difficult with M\&Ass.

Learning within strategic alliances is however a complex phenomenon (Inkpen 2002), and of course also has its problems and shortcomings. We will touch on the most obvious ones here. Firms must critically evaluate their partner's knowledge and the relevance of this knowledge for their own operations (Dyer and Singh 1998; Inkpen 1998). They should make sure that the partneting firm really has the desired knowledge, and that it is possible to get access to the knowledge via the proposed alliance. Partners in an alliance must also be willing to actively exchange knowledge, and be able to understand what they are learning (Hamel 1991). This thus also implies a capacity to learn and the appropriate processes and systems to facilitate learning (Inkpen 1998). Experience with earlier alliances can be helpful in successfully learning from later alliances (Westney, 1988) and experience with domestic alliances can be a stepping-stone to international alliances (Barkema, Shenkar et al. 1997). The management of international alliances is of course more difficult than that of domestic alliances. A last important point is the alignment of parent and alliance managers' culture, thereby avoiding that parent managers see the alliance as a threat and try to frustrate the alliances success (Schein 1996; Inkpen 1998). The largest problem in alliance learning is the loss of knowledge to the alliance partnet, and the accompanying threat of opportunistic behaviour by the partner. Trust is therefore a very important prerequisite for a successful alliance (Mody 1993).

Given these shortcomings strategic alliances are nevertheless frequently considered an effective means of accelerating the accretion of new capabilities. Via interorganisational learning, alliance partners can acquire resources or transfer knowledge. More in particular, alliances can play a major role in the efficient transfer of tacit knowledge. Whereas codified knowledge can be absorbed by studying e.g. a blueprint or recipe, without personal interaction, in 
the case of tacit knowledge interpersonal contact is very important (Nelson and Wintrer 1982).

Strategic technology alliances are therefore increasingly used for organisational learning and knowledge transfer (Kogut 1988; Hamell 1991; Parkhe 1991; Hagedoorn 1993b; Inkpen 1998; Sarkar, Echambadi et al. 2001). Such organisational learning can take on many different forms. We can distinguish among three main forms of organisational learning within strategic alliances (Inkpen and Dinur 1998). Firms make use of alliances to learn how to handle and manage future alliances. This type of learning primarily affects the managerial processes in the parent company. In this specific case no product or process knowledge is transferred. Knowledge might also be transferred merely for use in the present alliance operations. In this case no attempt is made to internalise the knowledge in the parent operations, nor was this the intent from the beginning. All the knowledge stays within the alliance itself. The third form of learning takes place when parent companies transfer the alliance knowledge to their own operations. Learning is primarily directed towards helping the parent companies to enhancing their own strategy and business operations. These three forms of learning obviously do not exclude each other. There might be combinations of these three forms of learning within alliances. Only when the last form of learning is included can we call it a learning alliance.

Various studies have argued that for effective learning processes in alliances, a sufficient degree of absorptive capacity is required. Cohen and Levinthal define absorptive capacity as the whole of the abilities of firms to use their prior related knowledge to walue external information, assimilate it and use it for their own commercial ends (Cohen and Levinthal 1990: 128). The absorptive capacity of a company is for a large part dependent on the current degree of knowledge in a specific technological field (Dodgson 1989; Cohen and Levinthal 1990; Levinthal 1994). Therefore we might argue that if a firm lacks a sufficiently developed technology base it is likely to have problems absorbing the newly acquired external technological knowledge. Alliance partners can only be expected to learn from the alliance as long as they have at least some prior knowledge in a specific field, so that they can incorporate the new knowledge and use it for their own means. Without an adequate degree of absorptive capaciry, a firm will not be able to learn. Firms will be better at internalising a partner's knowledge when they possess at least some overlap in knowledge bases (Mowery, Oxley et al. 1996; Lane and Lubatkin 1998; Lane, Slak et al. 2001). Too little overlap in knowledge bases between the allying firms is likely to inhibit learning, and therefore a minimal level of overlap in knowledge bases is necessary to facilitate learning (Ahuja and Katila 2001; Mowery, Oxley et al. 2002). On the other hand, when there is too much overlap there will be no learning either, because there is almost nothing the firms could learn from each other that they do not already know (Grant 1996). We can 
therefore expect that there is an optimal level of overlap, which will facilitate the learning the best. Therefore we hypothesize:

\section{$\mathrm{H}_{1}$ : The degree of overlap in the allying partners' initial knowledge bases has an inverted $U$-shaped relationship with the degree of learning taking place in the alliance.}

Although building absorptive capacity and a corresponding internal development of resources is important, learning from external sources is considered to be equally important for successful innovation (Cohen and Levinthal 1989; Cohen and Levinthal 1990; Vanhaverbeke, Duysters et al. 2002). In particular in turbulent high technology environments in which a firm's competitive position is determined by its abillity to innovate, alliances seem to be the most preferred option. Under conditions of change continued reliance on internally developed core competences makes firms extremely valnerable (Duysters and De Man 2002). Firms are therefore increasingly engaged in strategic technology alliances. Strategic technology alliances have enabled them to cope with the rising costs of R\&D efforrs and the speed and complexity of technological developments. An alliance with a competent partner enables firms to share development costs and to go faster down the learning curve. This might result in an improved time-to-market and a corresponding increase in the level of innovativeness. Because innovation has become one of the key competitive drivers, the use of alliances might therefore provide a means to achieve sustained competitive advantages. The effectiveness of strategic alliances for organisational learning is demonstrated by a recent study which showed that the most successful alliance firms are five times more likely to incorporate learning as an explicit goal of their alliances than their nonsuccessful counterparts (Accenture 1999).

The usefulness of strategic technology alliances for external learning of companies is tested in several studies (e.g. Mowery, Oxley et al. 1996; Lane and Lubatkin 1998). It turns out that strategic technology alliances ate a very effective vehicle for organisational learning. Learning via strategic technology alliances has many advantages. Alliances often enable firms to accelerate their capability development and helps them to reduce the time and risk involved in developing new products and technologies (Grant and Baden-Fuller 1995). Also the combining of knowledge of the firms involved in an alliance may prove to provide important synergistic effects, leading to new and better knowledge that neither of the partners could have realised independently. Furthermore, a set of alliances can often be seen as a radar function, which enables firms to explore new technologies developed by other companies. If one of these technologies proves to be successful the firm may choose to extent the alliance or to integrate the knowledge in-house. This decreases the 
risk of loosing out on new interesting technological opportunities and spreads the costs and risks among partners (Duysters and De Man 2002).

Besides establishing alliances for knowledge transfer, firms also form alliances in order to exploit their existing resources in new markets. In this case the alliance might not be a learning alliance, but rather a complementary alliance (Teece 1986). In a complementary alliance each partner brings in its own core competencies. One partner might have the knowledge of the market whereas the other might have the technical- or process knowledge. The aim of these kinds of alliances is not to share knowledge, but rather to complement the partner. Doz and Hamel (Doz and Hamel 1998) make the distinction between learning alliances and cospecialization alliances, where the former is aimed at learning from the alliance partner and the latter is primarily directed towards exploiting new markets.

The effect of a learning alliance on the relative post-alliance knowledge base overlap of the allying firms will be inversely related to the effect of a cospecialization alliance. A learning alliance can be expected to provoke an increase in overlap between the allying firms, because the intention of the alliance is to learn. For firms working together in a cospecialization alliance, one would expect no increase, or even a decrease in overlap, because firms will specialise in different technological fields and thus resemble each other less after the alliance. Also for firms that are not involved in an alliance we would expect a decrease, or at least no increase, in knowledge base overlap for the measuring period. Therefore the knowledge bases of allying partners in a learning alliance will show greater increase in overlap than do the knowledge bases of firms not involved in a learning alliance.

This leads to our second hypothesis:

$\mathbf{H}_{2}$ : Learning alliances will show significantly greater learning among the allying firms compared to firms that are not engaged in learning alliances, or not engaged in alliances.

In the social network literature the distinction between strong and weak ties (Granovetter 1973) has been posed to bear important implications on the nature of organisational learning. Weak ties are considered to be more important for the diffusion of unrelated knowledge whereas strong ties are more important for the diffusion of related knowledge. According to Granovetter the strength of a tie is " a combination of the amount of time, the emotional intensity, the intimacy, and the reciprocal services which characterize the tie" (Granovetter 1973: 1361) Firms connected via strong ties know each other well, and are also to some degree aware of the knowledge of the other partner. Firms connected through weak ties are usually less familiar with each other and with each other's knowledge base. In weak tie relationships, firms can 
learn from dissimilar knowledge bases whereas in the case of strong tes they can deepen their understanding of their existing knowledge. Weak ties are therefore more efficient as drivers of explorative research. They also tend to fulfil a bridge function between two, more or less unrelated business cliques, and are therefore geared towards combining previously distinct knowledge. "The lack of "social capital" (trust, comfort) is however likely to fuel opportuinism and a lack of commitment among the alliance partners. Strong ties on the other hand are used more often in exploitative research settings where firms from the same "clique" or technological field work together in order to deepen their existing knowledge. Therefore, we expect that the scope of the learning in a network comprised of weak ties is broader and in a network characterised by strong ties is deeper.

The degree of intimacy in the tie is related to the concept of trust (Gulati 1995). Before firms are willing to exchange information or knowledge they want to make sure that their sharing partner is trustworthy. Firms will be very wary of opportunistic behaviour, especially when the exchange touches on their core knowledge (Dollinger, Golden et al. 1997). According to transaction cost theory, the type of contact between firms depends on the anticipated transaction costs. Especially with core capabilities involved firms will be wery protective, and choose for a reliable partner. Also the resource-based theory of the firm considers reputation an important resource (Wernerfelt 1984). In combination with strong and weak ties we can expect that there will be note trust between partners with strong ties, than with partners who are connected via weak ties. As argued by Krackhard (1992: 218) these "... strong ties constitute a base of trust that can reduce resistance and provide comfort in the face of uncertainty". In strong ties, opportunistic behaviour affects the reputation of firms more than in a situation of weak ties. If a firm is considered a non-trustworthy partner in a network of strong ties this news will travel quickly, and its effect on the opportunistic firm will be considerable. In a weak tie situation both problems are less critical, and might even be outweighed by the application of the knowledge gained in the own clique. We can expect firms to work together in weak ties only with their peripheral competencies, which they want to expand. For their core competencies they will relay on strong ties.

So while furms could potentially learn a great deal from weak tie contacts, the fear for opportunistic behaviour, and consequently the lack of trust might inhibit knowledge flows. Although learning in a strong tie situation will be less broad, we can expect more knowledge flows in these kinds of interactions due to greater trust between partners. This leads us to expect that most of the observed knowledge flows will be between firms connected via strong ties. We would therefore expect more learning taking place in a strong tie alliance as opposed to a weak tie alliance. 
Therefore our third hypotheses:

\section{$\mathrm{H}_{3:}$ The learning, taking place in strong tie alliances is larger than in weak tie alliances.}

\subsection{Data}

Our sample of firms is taken from the Fortune 500 list in 1997 (for a more complete description of the database construction see chapter 2). We selected all firms in the medium- to high tech sectors. This provided us with a set of 171 parent firms in 12 sectors. Using the Dun \& Bradstreet Linkages dataset we searched all the subsidiaries of these 171 firms to construct a "group" list per firm. "This "group" list makes it possible to trace back more of the patents of the multinationals we studied. This enables us to include the patents and patent citations of their subsidiaries, and not just those of only the parent company. The version of the Dun \& Bradstreet Linkages database that we used originates from 1998, and thus represents the "group" list at that particular moment in time, or actually a bit earlier. Of course, the parent subsidiary relationships will change over time, so we want our measuring moment to be as close as possible to the moment of the sample construction.

For the data on patents we made use of the European Patent Office (EPO) data set. Based on the "group" lists we constructed, we searched the EPO data set for all patents of the multinational. In the EPO database the patents are recorded by applicant name. Sometimes we found names that only partly corresponded with the names of the firms we were looking for, in that case we compared the address we got from Dun \& Bradstreet with the address contained in the EPO database. If they were identical we included the patents in our sample, otherwise we excluded them. We also used the patent citation data present in EPO so that we could end up with a list of all the patent citations per patent.

We made use of the well-known MERIT-Cooperative Agreements and Technology Indicators (CATI) database for the information on alliances. The CA'T database is a relational database containing over 15,000 cooperative agreements involving about 9500 firms. Systematic collection of inter-firm alliances started in 1987, but earlier years were searched in retrospect. Different sources were used for the construction of the database, among the most important are newspapers and trade journal articles. Even though the dataset will be inevitably incomplete, and biases might be present, CATI is the most complete and dependable source available on cooperative agreements.

For data on R\&D expenditures and number of employees we made use of the Worldscope clatabase. The data on $R \& D$ expenditures was converted to U.S. Dollars to facilitate comparison. 


\subsection{Methods}

The knowledge that a firm possesses can be thought of as residing in the patents owned by the firm. Patents are by definition representations of new and unique pieces of knowledge, and as such, the collection of patents a firm has, represents its total set of knowledge. Following Ahuja and Katila (2001), we also include the patents the furm is citing in its own patents; for also the knowledge included in these patents mast, to some extend, be known to the firm. Even though the firm itself, just for legal reasons, might include some of the patent citations, or they might be included by the patent officer reviewing the patent, these citations indicate a knowledge relationship, and the firm can be expected to have at least some idea of the knowledge involved, especially at the multinational level. The general knowledge base of a firm in our sample is then defined as the total of own patents of the firm, plus the patents cited in these patents. The individual patents in each firm's knowledge base can than be compared with the patents in other firms' knowledge bases.

Patent-based measures of course have their limitations see for instance Griliches (1990), and chapter 2 of this thesis; the propensity to patent for instance might differ per industry. Some industries rely heavily on patents while others do not, we therefore will include a dummy variable for alliances between firms from different industries, and alliances between firms from the same industries. Furthermore, alliances are especially important for the transfer of tacit knowledge (Badaracco 1991), but the patents we are using are by definition examples of codified knowledge. This could lead us to exclude the tacit knowledge component from our analysis. The tacit knowledge flowing between firm boundaries, however, is almost impossible to measure, but there is substantial evidence that tacit knowledge flows are closely linked with codified knowledge flows (Patel and Pavitt 1997), and thus we feel confident in the use of patent data.

We started our analysis by extracting alliance pairs for 1993, our base year, from the CATl database, that belong to our set of medium- to high tech firms from the Fortune 500 list of 1997 . For these alliance pairs we calculated or collected the necessary variables. We found 78 unique alliance pairs, which we used for our analyses.

We used 1993 as our base year for two reasons. The first reason to do this is because the year 1993 lies close to 1998, the year of our database construction. The closer we are to 1998 the more confident we can be that the results we find can be extrapolated to the 1998 configuration of firms and interconnections. Furthermore taking 1993 as a base year still gives us enough measuring years to be able to retrieve reliable information from our data. 


\section{Dependent variable}

As our dependent variable we took the Knowledge Base Overlap after the alliance was established $(\mathrm{KBO})$. The Knowledge Base Overlap is defined as the number of patents that appear in both firms" general knowledge bases, divided by the total number of patents in both firms' general knowledge base. We measure this overlap for the five years after the establishment of the alliance, so for 1994 till 1998. This has to do with the time it takes to obtain a new patent and the time to undertake subsequent patent citations. So the Knowledge Base Overlap for a certain alliance between Firm $i$ and Firm; after the alliance is:

$$
K B O_{A i j}=\left(K B_{A i} \cap K B_{A_{i}}\right) /\left(K B_{A_{i}} \cup K B_{A i}\right)
$$

\section{Independent variable}

Our independent variable is the Knowledge Base Overlap before the alliance is established $\left(K B O_{B}\right)$. Here we measure the overlap in the firm's general knowledge bases in the five years before the establishment of the alliance. The Knowledge Base Overlap for the alliance between Firm ${ }_{\mathrm{i}}$ and Firmi before the alliance is defined as:

$K \mathrm{KO}_{\mathrm{Bij}}=\left(\mathrm{KB}_{\mathrm{Bi}} \cap K \mathrm{~B}_{\mathrm{Bi}}\right) /\left(\mathrm{KB}_{\mathrm{Bi}} \cup \mathrm{K} \mathrm{B}_{\mathrm{Bi}}\right)$. We will use $K \mathrm{KOO}_{\mathrm{Bij}}$ as well as $\left(\mathrm{KBO}_{\mathrm{Bii}}\right)^{2}$.

\section{Control variables}

We also include the number of prior alliances of the firms $\left(P A_{G i i}\right)$ in general, so with all other firms it allied with, and the number of prior alliances "special" thus with the same orher firm $\left(\mathrm{PA} \mathrm{A}_{\mathrm{Si}}\right)$, as independent variables. For both variables we looked at the alliances the firms had since 1970 and for the five successive years before the alliance, and used the average of both firms in the alliance. "This provides us with four variables $P A_{G i j} 70, P A_{S i j} 70, P A_{G i j} 5$ and $P A_{\text {sij }} 5$. We expect that the number of prior alliances will have a positive influence on the learning taking place. Firms that work together more often will experience more trust within the relationship, so this might increase the learning. On the other hand, firms that had more alliances will have more experience in dealing with an alliance and also this will increase the likelihood of a knowledge transfer. We expect thus a positive influence from the number of prior alliances on the learning.

To further test the influence of strong and weak ties in a relationship we also used the number of Prior Equity Alliances (PEAs5) with the same other firm in the five years before the alliance as a proxy of the strength of the tie 
between the two firms in the alliance. In an equity alliance the allying firms have strong commitments to each other, inhibiting opportunistic behaviour. Nonequity alliances on the other hand have more characteristics of the looser relationship of a weak tie. The more equity alliances two firms have prior to the measuring alliance of 1993, the stronger we can expect their tie to be. We allso included a dummy variable (EQUITY), which is tepresented by a one (1) in the case of an equity alliance in 1993 and a zero (0) for a non-equity alliance between the two firms in 1993 . We included this variable to correct for a possible influence of the current form of the alliance.

Other control variables we included are the logarithm of $R \& D$ spending of the firms in the alliance $\left(R \& D_{i j}\right)$. If firms dedicate a larger amount of spending to R\&D they will have more in-house knowledge to process the new knowledge. Furthermore, these firms are likely to have a learning attitude. Further we include the Logatithm of the number of employees (SIZE $\mathrm{E}_{i j}$ of the allying firms. Size is likely to have a positive effect on knowledge flows. A larger firm can be expected to have a larger pool of knowledge to draw from, and will thus be better at incorporating new knowledge. Also bigger firms have more resources for incorporating the new knowledge. On the other hand, smaller firms are usually considered more innovative than big firms, which would lead us to expect less learning in bigger firms. For both variables, $R \& D_{i \mid}$ and $S I Z E_{i j,}$ we use the average values of both firms in the alliance.

We also included a dummy variable for firms from the same industry allying $(\mathrm{SECTOR}$ ). We might expect more learning taking place between firms from the same industry, because the knowledge overlap between the firms will be bigget. On the other hand, because of competition sensitivities firms might be more reluctant to share knowledge with firms from the same industry. We though expect the first influence to be more influential. This means that it will be represented by a zero ( 0 ) if two firms from different industries are allying and by a one (1) if they are from the same industry.

To test our hypotheses we test the following empirical specification:

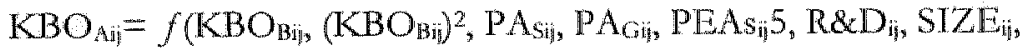

$$
\begin{aligned}
& \text { SECTOR, EQUITY, }
\end{aligned}
$$

The testing of Hypothesis 2 requires the construction of a control group. To be able to test if firms working together in a learning alliance learn mote than firms who are not involved in such an alliance, of not allying, we needed to construct a control group of firms who had not worked together in a learning alliance. Using the CATI-database we searched for every alliance pair $A-B$ in our dataset a firm $C$ that did not have an alliance with neither $A$ not $B$ 
and that resembled $B$ as close as possible, concerning industry, firm size, R\&D spending and number of patents. The "new" firm $C$ was than put together with the "old" firm A. This "matched" pair A-C was used as control group in the testing of hypothesis 2 .

\subsection{Results}

Before we can start testing our hypotheses we first take a closer look at our data, and at its specific characteristics. The results are reported in table 4.1.

\begin{tabular}{|c|c|c|c|c|}
\hline Variable & Megn & Std. Dev. & Min. & Max. \\
\hline $\begin{array}{l}1 K B O A^{5} \\
(x \| 000)\end{array}$ & 4.45 & 5.11 & 0 & 30.43 \\
\hline $\begin{array}{c}2 \times B O_{13} 5 \\
(x 1000)\end{array}$ & 4.36 & 5.44 & 0 & 26.40 \\
\hline $\begin{array}{c}3\left(K B O_{B} 5\right)^{2} \\
(\times 1000)\end{array}$ & 48.22 & 108.86 & 0 & 696.93 \\
\hline $4 P A_{\text {Sili }} 70$ & 1.60 & 1.86 & 0 & 7 \\
\hline $5 P A_{S i n} 5$ & .92 & 1.49 & 0 & 7 \\
\hline $6 \mathrm{PA}_{\mathrm{G} i \mathrm{j}} 70$ & 68.12 & 41.32 & 5 & 182.50 \\
\hline $7 P A_{6 \mathrm{ij}} 5$ & 32.94 & 23.43 & 1 & 85.50 \\
\hline $8 P E A s_{1 i}^{5}$ & .29 & .65 & 0 & 3 \\
\hline$g R \& D_{i j}$ & 6.99 & 0.93 & 4.77 & 8.71 \\
\hline $10.512 E_{3 j}$ & $\| 1.76$ & 0.64 & 10.48 & 13.09 \\
\hline $11 \mathrm{SECTOR}$ & .35 & .48 & 0 & 1 \\
\hline 12 EQUITY & .24 & .43 & 0 & 1 \\
\hline
\end{tabular}

For the testing of hypotheses 1 and 3 , on the relationship between prior knowledge and alliance learning, and the influence of strong and weak ties in alliances on learning, we test our model using regression analysis. Since our dependent variable is left censored (see figure 4.1) we cannot use standard OLS regression, but instead have to use Tobit regression. 


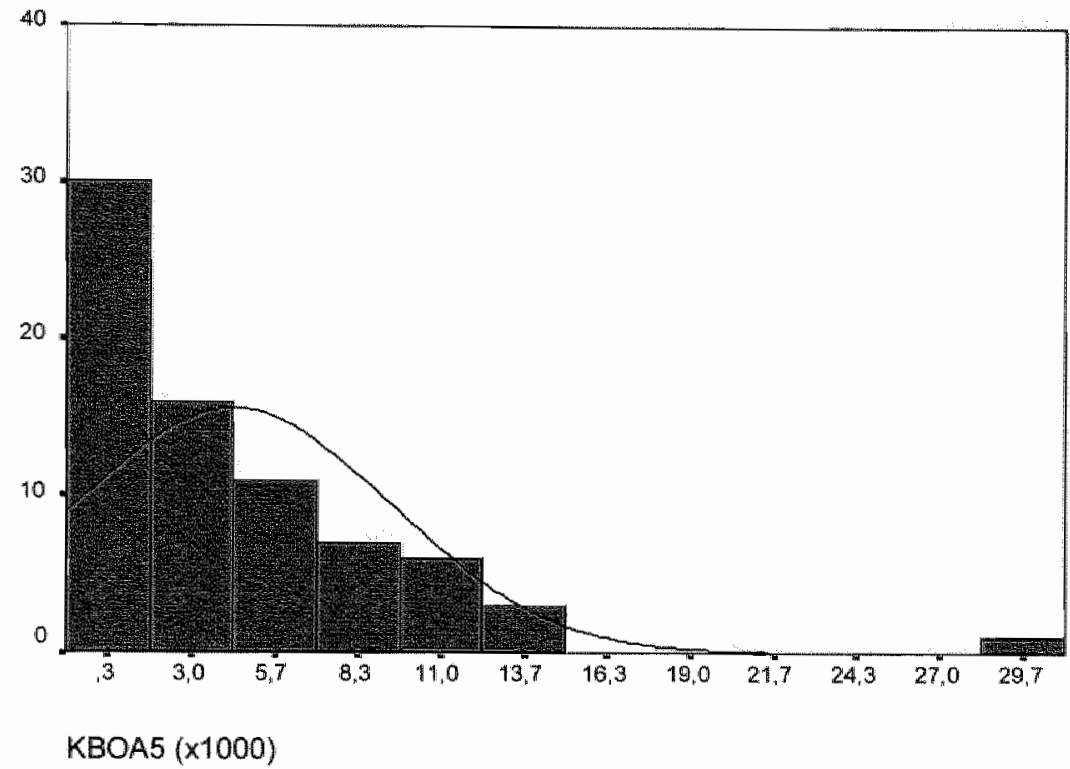

Figure 4.1 Spread of the dependent variable $K \mathrm{BO}_{4} 5$

Table 4.2 shows the correlations for hypotheses 1 and 3 . It turns out that there are no severe correlations among our independent variables, except for $\mathrm{R} \& \mathrm{D}_{\mathrm{ij}}$ and firm size measured as the logarithm of the number of employees $(0.758)$, and between one of the PA $A_{G i}$ variables $\left(P A_{G} 70\right)$ and both $R \& D_{i j}(0.817)$ and SIZE $(0.710)$. We ran our regressions with different combinations of these variables, and it turns out to make no difference for our results. The same applies to $P A_{G} 5$ and $R \& D_{i j}(0.742)$. Some other high correlations are among variables that were never regressed together, thus posing no problem. The different Prior Alliance variables for instance are highly correlated, which is logical, but we only use one of these variables in our regressions at a time. We also regtessed every independent vatiable on all the other independent variables (values not reported here); this shows no serious multicollinearity among the independent variables. All the VIF values were well below 5 (were 10 is the standard cut-off value). 
Table 4.2: Correlations for the variables in hypotheses 1 and 3 .

\begin{tabular}{|c|c|c|c|c|c|c|c|c|c|c|c|}
\hline Variable & 1 & 2 & 3 & 4 & 5 & 6 & 7 & 8 & 9 & $10 \quad 11$ & 12 \\
\hline $1 \mathrm{KBO} / 5$ & 1.00 & & & & & & & & & & \\
\hline $2 K B O M$ & $.7390+4$ & 1.00 & & & & & & & & & \\
\hline $3\left(K B O_{B} 5\right)^{2}$ & $.628^{*}$ & $4.918^{8}$ & $* 1.00$ & & & & & & & & \\
\hline $4 P A_{y y} 70$ & .321 ** & $3.349 \%$ & 245 & 1.00 & & & & & & & \\
\hline $5 P A w_{j}^{5}$ & .181 & $.288^{8+4}$ & .204 & $.793^{*}$ & 1.00 & & & & & & \\
\hline $6 P A_{G i j}>0$ & .305 * & $* .410^{* x}$ & $350^{\text {thx }}$ & $* 470 *$ & $.3599^{*}$ & N" 1.00 & & & & & \\
\hline $7 P A_{C, 5}$ & $.356^{* * 2}$ & $447^{\text {kn }}$ & $392^{\text {水 }}$ & $* 466 *$ & . 402 & $* .939 * *$ & $* 1.00$ & & & & \\
\hline $8 P E A s_{i j}$ & .027 & .163 & .134 & $.541^{*}$ & $* 710^{* * 4}$ & $* 257 *$ & $.253 *$ & 1.00 & & & \\
\hline $9 R C^{\prime}$ & $.299 *$ & *. $405^{*}$ & 338 & $448 *$ & $400^{*}$ & 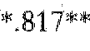 & .742 & $.331 *$ & $* 1.00$ & & \\
\hline $1.0 S I Z E_{i / Z}$ & .180 & $224^{*}$ & .202 & $.355 *$ & $334 * 3 *$ & 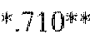 & $* .552$ * & $.288 *$ & $758 *$ & 1.00 & \\
\hline $11 S E C T O$ & $R_{i, \pi} 413^{*}$ & $* .311 *$ & $.289^{\text {\% }}$ & .171 & .201 & -.018 & -.018 & $.253^{*}$ & .038 & .0591 .00 & \\
\hline 12 EQUTT & $3 y-054$ & -.076 & -.067 & .025 & -.051 & .037 & -.025 & -.028 & -.004 & $.075-162$ & 1.00 \\
\hline
\end{tabular}

Table 4.3 provides the empirical results for the Tobit regression for hypotheses 1 and 3 . We only show those regressions that we ran with different combinations of variables that give extra information, more regressions were carried out but they gave no different results. As extra control we also regressed using OLS regression. The results are in line with the results as reported under Tobit regression. $\mathrm{KBO}_{\mathrm{Bii}}$ turns out to be very significant every time we ran the regression, and the sign is always positive. Our independent variable $\left(\mathrm{KBO}_{\mathrm{Bii}}\right)^{2}$ is also significant in every regression and this time the sign is always negative. 
Put together these two variables provided strong proof for our first hypothesis; that there is an inverted $U$-shaped relationship between the learning taking place in an alliance and the prior knowledge overlap between the firms. To further test this relationship we investigated the shape of this relationship, using the first regression in table 4.3 . We plotted the relationship between $\mathrm{KBO}_{\mathrm{B}} 5$ on the $X$-axes and the learning effect on the $Y$-axes (see figure 4.2). This gives us the gross learning effect.

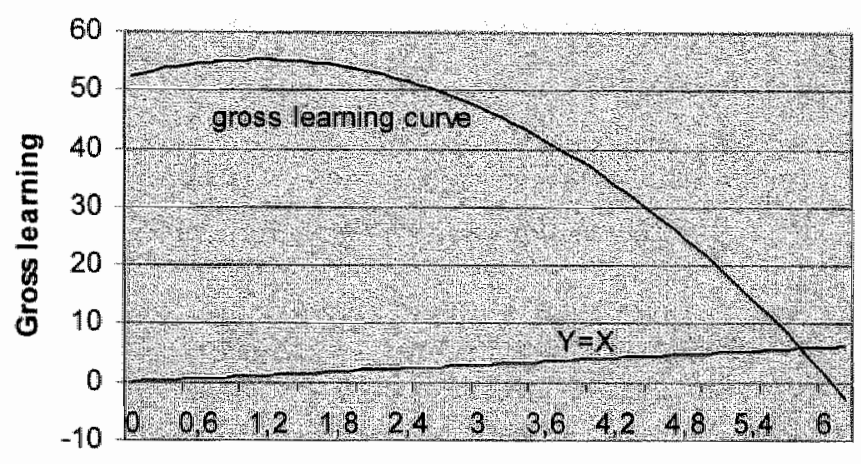

Knowledge base overlap before KBOb5

Figure 4.2 Gross learning effect

On the line $Y=X$ both $K B O_{A} 5$ and $K B O_{3} 5$ have the same value and no learning is taking place. Learning takes place for the part of our parabola that is above the $Y=X$ line. If we thus want to know the net learning effect we need to subtract the line $\mathrm{Y}=\mathrm{X}$ from our parabolic relationship. By doing this we end up with a new parabola with a maximum for $\mathrm{KBO}_{\mathrm{A}} 5$ at $\mathrm{KBO}_{\mathrm{B}} 5=0.9$ (see figure 4.3). The formula of the parabola is $-2.253 \mathrm{X}^{2}+4.062 \mathrm{X}+52.42$ : the control variables are added up to give one value for the constant. The parabola crosses the $X$-axes at $X_{1}=-4.01$ and $X_{2}=5.81$. Since our dependent variable has a mean of 4.45 and a standard deviation of 5.11 we know that $95 \%$ of our results are in the area of $X=4.45 \pm(2 \times 5.11)$, thus from $X=-5.77$ till $X=14.67$. Our tesults are well within this range, and the net learning does indeed show an inverted $U$. shaped relationship, where the learning first increases with increasing before 
knowledge base overlap $\left(\mathrm{KBO}_{\mathrm{B}} 5\right)$, reaches a maximum for $\mathrm{KBO} 5=0.9$, and decreases after this point.

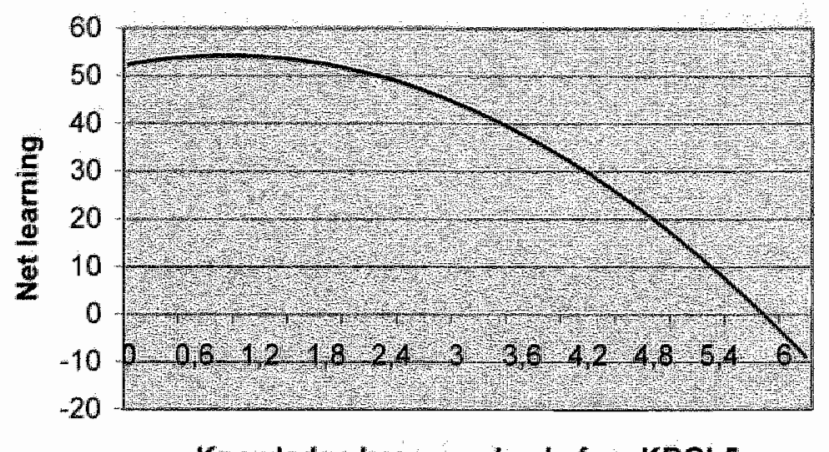

Figure 4.3 Net learning effect

Looking further at table 4.3 it turns out that also our control variable SECTOR $_{\mathrm{ij}}$ is significant and positive for every regression analysis. This indicates that indeed there is more learning taking place in an alliance between firms from the same sector, compared to firms from different sectors.

Hypothesis 3 is not supported by our data. We find on the contrary strong evidence for the opposite, weak ties are more important for learning than strong ties. Out variable PEAs5 is every time significant and negative. This thus indicates that more equity alliances with the same firm leads to less learning. Our Prior Alliance variables all give positive results though not always significant. Thus the number of prior alliances has a positive influence on the learning in strategic alliances. These two results combined supports strong evidence that the alliances in our sample learn more from weak ties than from strong ties. It could be that complementarity outweighs trust for the firms in our sample. Another explanation for this remarkable result might be that the firms are suffeting from "over-embeddedness" (Uzzi 1997). Embeddedness influences the firms" allying behaviour (Granovetter 1992; Gulati 1998), leading to preferential allying partners, since trust is an important basis for knowledge 
exchange and partner selection (Cyert and March 1963; Nelson and Winter 1982; Mody 1993). This would lead firms to search for allying partners among their trusted partners (Stuart and Podolny 2000) with whom they have had beneficial partnerships in the past. This reduces search costs and alleviates opportunistic behaviour between the partners (Gulati and Gargiulo 1999). The more firms rely on these same partners, with whom they get strongly connected since they have frequent interactions, with this same partner and will commit a great deal to the relationship, the more they are going to resemble the partner and the less they are able to learn from it. When partners become more familiar with each other, they start to resemble each other more (Friedkin 1984). The proximity between partners reduces the divergence of the attitudes berween the partners, especially for partners who are connected via strong ties (Brass, Butterfield et al. 1998). They might develop core rigidities (Leonard-Barton 1995), which can cause them to fall into competency traps (Levitt and March 1988). Alliance firms thus get isolated from possible alliance partners outside the current alliance; therefore they will suffer from decreasing possibilities for learning and innovation (Duysters and Lemmens 2003). The more firms work together, the greater the trust and intimacy between them will grow (Bass, Butterfield et al. 1998). Over time this may lead to "over-embeddedness" where firms get too similar, decteasing opportunities for learning and innovation (Duysters, Hagedoorn et al. 2003). Learning via strong ties is thus still beneficial and very important for the allying firms, but research suggests that there is a limit to the positive effects observed. The stronger firms work together and especially the longer they work together in stronger ties, the less their innovative performance, and the less they can learn from each orher (Duysters, Hagedoorn et al. 2003). This outcome is inline with our findings and serves ats a good explanation for the results we are finding.

We find slight positive results for our control variable EQUTT'Y (but never significant), indicaring that the current form of the alliance is impottant for learning. We also ran the regressions without this control variable and it turned out to make no difference for our results. 


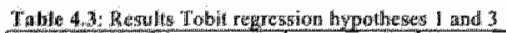

\begin{tabular}{|c|c|c|c|c|c|c|c|}
\hline 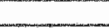 & (1) & (2) & (3) & (4) & $(5)$ & 60 & (7) \\
\hline $\begin{array}{l}1 \% B O, 5 \\
1 \times / 600)\end{array}$ & $\begin{array}{l}5.662^{2 m+t} \\
60.2093)\end{array}$ & $\begin{array}{l}5.0909 \% 00 \\
(0.099)\end{array}$ & $\begin{array}{l}4.833^{104} \\
(0.204)\end{array}$ & $\begin{array}{l}5.158^{6 / 4} \\
(0.203)\end{array}$ & $\begin{array}{l}5.2196 \% \\
(0.206)\end{array}$ & $\begin{array}{l}4.876^{\circ} \text { o } \\
(9.210)\end{array}$ & $\begin{array}{l}5.351^{* * *} \\
(0.204)\end{array}$ \\
\hline $\begin{array}{l}\left(K \mathbb{8} O_{15} 5\right)^{2} \\
(\mathrm{~N} / 000)\end{array}$ & $\begin{array}{l}-2.253^{* 6} \\
0.0101\end{array}$ & $\begin{array}{l}-2.287^{618} \\
(0.009)\end{array}$ & $\begin{array}{l}-4.87 \% \\
0.0169\end{array}$ & $\begin{array}{l}-2.236 \% * \\
(0.010)\end{array}$ & $\begin{array}{l}-2.11 \text { 19.th } \\
(0.610)\end{array}$ & $\begin{array}{l}2.130^{* *} \\
(0.010)\end{array}$ & $\begin{array}{l}-2.165^{*} \\
(0.010)\end{array}$ \\
\hline$P_{A_{s i}} 70$ & & & $\begin{array}{l}2.044^{\text {"1* }} \\
(0.285)\end{array}$ & & & & \\
\hline$\beta_{A_{3 j} j^{5}}$ & & & & & $\begin{array}{l}0.707 \\
(0.415)\end{array}$ & & \\
\hline$P A_{i t i l}>0$ & & & & $\begin{array}{l}1.481 \\
(0.013)\end{array}$ & & & \\
\hline$\hat{\beta}_{A, i j j^{\prime}}$ & $\begin{array}{l}1,945 \\
(0.023)\end{array}$ & $\begin{array}{l}1984+3 \\
(0.022)\end{array}$ & & & & $\begin{array}{l}0.467 \\
(0.023)\end{array}$ & \\
\hline$P E A s_{i j} 5$ & $\begin{array}{l}-2,323 \% \\
(0.65 \%)\end{array}$ & $\begin{array}{l}-2,361 * \\
(0.671)\end{array}$ & $\begin{array}{l}.2 .767^{* *} \\
(0.756)\end{array}$ & $\begin{array}{l}-2.22)^{\prime} \\
(0.676)\end{array}$ & $\begin{array}{l}-1.862 * \\
(0.921)\end{array}$ & & $\begin{array}{l}-1.898^{*} \\
(0.660)\end{array}$ \\
\hline$R d D_{i f}$ & $\begin{array}{l}-1.782^{*} \\
(0.143)\end{array}$ & & & & & $\begin{array}{l}-1.709^{*} \\
(0.147)\end{array}$ & $\begin{array}{l}-0.598 \\
(0.101)\end{array}$ \\
\hline$S I Z R_{i j}$ & & $\begin{array}{l}-1.918 \% \\
(0.01509)\end{array}$ & $\begin{array}{l}.1 .300 \\
(0.062)\end{array}$ & $\begin{array}{l}\times 1.619 \\
(0.087)\end{array}$ & $\begin{array}{l}.0 .908 \\
(0.061)\end{array}$ & & \\
\hline$S E C T O R_{i f}$ & $\begin{array}{l}3.972^{2 * 4 *} \\
(0.865)\end{array}$ & $\begin{array}{l}4.052^{* * *} \\
(0.865)\end{array}$ & $\begin{array}{l}3.722 * * * 1 \\
(0.823)\end{array}$ & $\begin{array}{l}3.831^{* * *} \\
(0.876)\end{array}$ & $\begin{array}{l}3.577^{* * *} \\
(0.842)\end{array}$ & $\begin{array}{l}3.562 * 110+1 \\
(0.824)\end{array}$ & $\begin{array}{l}3.474 * 4 * \\
(0.834)\end{array}$ \\
\hline EQUTTY & $\begin{array}{l}0.364 \\
(0.813)\end{array}$ & $\begin{array}{l}0.410) \\
(0.810)\end{array}$ & $\begin{array}{l}0.214 \\
(0.802)\end{array}$ & $\begin{array}{l}0.260 \\
(0.818)\end{array}$ & $\begin{array}{l}0.366 \\
(0.828)\end{array}$ & $\begin{array}{l}0.360 \\
(0.832)\end{array}$ & $\begin{array}{l}0.220 \\
(0.823)\end{array}$ \\
\hline
\end{tabular}

To test our second hypothesis we used two different methods. First we used a t-test to see if there was a difference between the two groups, the alliance group and the control group. Since our data is not fully normally distributed we used a Wilcoxon Signed Ranks test, next to a normal t-test, to compare the two groups. We find that our groups are significantly apart at the $5 \%$ significance level for both the normal t-test and the Wilcoxon Signed Ranks test. The increase in learning is significantly greater for the firms from the alliance group as compared to the firms from the control group. The results are reported in table 4.4 . 
Table 4.4: Results t-test and Wilcoxon Signed Ranks test.

\begin{tabular}{|l|l|l|l|l|}
\hline & Mean & Sid. Dev. & Sig.(2-lailed) \\
\hline T-hest & 0.972 & 4.601 & 1.865 & 0.066 \\
\hline & & & $Z$ & Sig.(2-tanled) \\
\hline Wilcok. Sign. R. & & & -2.176 & 0.030 \\
\hline
\end{tabular}

$N=78$

As a second test for the difference we regressed the Knowledge Base Overlap before and after of the two groups, the alliance group and the control group, using a dummy variable (ALLIACon). The dummy variable is zeto (0) if the firms belong to the alliance group, and one (1) if they belong to the control group. It turns out that the dummy variable is significant and negative, indicating that the firms in the alliance group learn significantly more than the firms in the control group (for results see table 4.5). It turns out that alliances are an important vehicle for learning among firms.

Table 4.5: Regression results hypothesis 2 .

\begin{tabular}{|l|l|}
\hline Variable & \\
\hline $\mathrm{KBO}_{B} 5$ & $0.743^{* * * *}$ \\
$(\mathrm{x} 1000)$ & $(0.041)$ \\
\hline ALLIACon & $-0.183^{* * * *}$ \\
& $(0.438)$ \\
\hline
\end{tabular}

$* * *=p<0.01$. Standard errors in parentheses 


\subsection{Discussion and Conclusions}

In this chapter we empirically investigated the effect of strategic technology partnering on the knowledge bases of companies. From a knowlledge-based perspective we hypothesised that the degree of overlap in the allying partners initial knowledge base is inverted U-shaped related to the degree of learning taking place in the alliance. Our empirical results indeed show that a medium degree of knowledge overlap between alliance partners is more effective than a degree of knowledge overlap that is either too high or too low. This supports the existing literature on absorptive capacity, which argues that if firms have too little overlap in terms of their technological know-how they will be unable to absorb the know-how of their partners. On the other hand, if firms are too similar they might suffer from a lack of synergy in the alliance. If similar players are linked in an alliance chances increase that the information flows between partners are redundant (Burt 1992; Krackhardt 1992).

Our second hypothesis argued that firms engaged in learning alliances will show higher degrees of learning than firms not engaged in these alliances. The results from our analysis show that alliances can be seen to have a significant. and positive effect on the learning rate of the companies in our study. This might be surprising given the high failure rates of strategic alliances that can be found in the literature (for an overview see (Duysters, Kok et al. 1999). The finding is however in line with more recent work in the area of innovation studies (Mowery, Oxley et al. 1996; Powell, Koput et al. 1996; Lane and Lubatkin 1998; Anand and Khanna 2000). This body of literature shows that learning alliances seem to be a particularly effective means of knowledge acquisition.

Our third and final hypothesis was concerned with the differences in learning rates of strong versus weak ties. We argued that strong ties would be more effective in transferring technological know-how because firms are more familiar with each other and will show higher trust levels. As a result, the chances of opportunistic behaviour between partners are considered to be lower and therefore we expect that information will flow more effectively between partners. Our findings however indicate that weak ties are more effective than strong ties. This seems to suggest that complementarity outweighs trust in alliance relationships. Synergetic effects might be higher in weak ties than in the case of strong ties. Furthermore, new knowledge generated in weak ties is likely to be more innovative than knowledge that is generated in strong ties relationships. On the other hand, firms connected via strong ties might also suffer from "over-embeddedness", leading them to develop core rigidities, and decreasing the learning potential from their partner. 
Therefore, the chances that knowledge exchange leads to the application of patents is likely to be higher in weak tie relationships.

Overall, we can conclude that alliances have established themselves as an important means of (external) knowledge acquisition but that partner selection forms at critical determinant for the effectiveness of the knowledge exchange process. In this partner selection process the knowledge overlap between the allying firms, and the strength of their tie seem of eminent importance. 


\section{CHAPTER 5 \\ The Spatial Dimension of Patenting by Multinational Firms in Europe}

\section{This chapret is based on the article Verspagen, B and W. Schoenmakers (2004). The spartal dimension of parenting by multinational. firms in Europe: " Louthal of Econonic Geograp hy $4(1), 23-42$}

\subsection{Introduction}

Even though the notion of globalisation has often been used without a very clear and precise definition, it has been argued that this development makes the role of space disappear (see Morgan 2004). The essence behind this argument is that with modern communication technologies, interaction between organizations and people can be effectively implemented over large distances, and hence physical presence is no longer a prerequisite to interact with a region's economic, social and technical system.

At the same time, it has been argued that technology and knowledge generation is an exception to the globalisation trend. For example, Patel and Pavitt (1991), in an analysis of patenting by the world's largest multinational enterprises (MNEs), conclude that technology is an important case of "nonglobalisation". By this, they mean that the MNEs in their sample undertake the largest part of their $\mathrm{R} \& \mathrm{D}$ activities in their home base country. However, even their analysis, as well as subsequent evidence provided by, e.g. Le Bas and Sierra (2002) indicates that although the largest part of R\&D by MNEs is performed in the home base, the part that is done abroad is non-negligible. We are thus left with the impression that R\&D activities by MNEs are subject to "globalisation", but to a lesser extent than other activities, such as production and marketing by the same firms.

Does this imply the "death of geography" in innovation systems, as the paper by Morgan asks? "The analysis here will argue exactly the opposite, i.e., that the tendency to perform R\&D abroad implies a strengthening of the notion of regional innovation systems. The line of reasoning will test on one important argument, namely that due to the existence of specific skills and competencies in people who are not perfectly mobile, technological capabilities of specific regional innovation systems cannot be tapped into easily from a distance. Thus, an MNE wishing to make use of such specific knowledge will have to acquire presence in the region, either by setting up a Greenfield R\&D 
facility, or by taking over an existing firm well embedded in the region's innovation system.

This is not a new vision on the role of regional innovation systems as a continuing factor even in the days of Intemet. Consequently, the chapter's contribution is not aimed at providing a new theoretical argument to support this vision. Instead, the chapter aims at providing some empirical evidence in support of the importance of regional innovation systems in Europe. For this purpose, it uses an extensive database on patenting by the world's largest MNEs from European locations. To our knowledge, this chapter presents the first European regional analysis of patenting activities by the world's largest firrins, based on European patents. ${ }^{3}$

The rest of the chapter is organized as follows. The next section will provide a short overview of the main theoretical starting points for our empirical analysis. The main parts of the literature that will be surveyed are the business literature on foreign R\&D activities by MNEs, and the economics and geographical literature on the local nature of knowledge and knowledge flows. Section 5.3 will present our database, and discuss the way in which we implement our indicators. Section 5.4 provides an overview of how $R \& D$ activity by the firms in our sample is spread over Europe's regions. In section 5.5 we will explore the reasons for the observed spread of $R \& D$ activities over the European regions and discuss the stability of this distribution over time. Section 5.6 will go deeper into the issue of knowledge flows, by using patent citations indicators. Finally, section 5.7 will provide the main conclusions.

\subsection{Theory and hypotheses}

The empirical research in this chapter will investigate two main hypotheses. The first is that there is now ample reason for multinational enterprises (MNEs) to locate at least part of their R\&D activities outside the home country. The second is that for choosing in which (foreign) region to locate these R\&D activities, a limited number of regions will be favoured more than other regions. This section aims to provide a concise survey of the existing literature dealing with these topics. As will be seen in the discussion, the two questions are not necessarily the same. Although from a geographer's point of view, it might seen only too obvious that R\&D activities cluster regionally, the business literature dealing with locational behaviour of $R \& D$ activities of MNEs does not necessarily come to this conclusion.

\footnotetext{
5 Previous works using a regional database of European patents, such as Brescliu (2000), Caniëls (1999) and Maurseth and Verspagen (2002) have not used data at the firm level.
} 
This business literature (e.g., Dunning and Narula 1995; Cantwell and Janne 1999; Patel and Vega 1999) has only recently come to the conclusion that "globalisation" is an important phenomenon affecting $R \& D$ activities of MNEs. Patel and Pavitt (1991) saw foreign R\&D activities of MNEs taking place, but concluded that foreign R\&D of these firms was by-and-large an important case of "non-globalisation". Subsequent literature has indeed concluded that foreign R\&D is on the rise. For example, Le Bas and Sierra (2002: 600), on the basis of patent data for 350 large firms known to be strong innovators, conclude that for the period 1994-1996,19.5\% of their total patents stem from R\&D performed outside the home country of the firm. The number was $15.8 \%$ for the period 1988-1990. They also report cases where up to $60 \%$ of all patents stems from foreign research.

The theoretical explanation for this trend points to two motives for locating $R \& D$ abroad. The first one can be called asset-exploiting foreign $R \& D$ (Dunning and Narula 1995). In this case, firms seek to exploit their existing technological capabilities (developed by home base R\&D) by means of performing $R \& D$ that is aimed at adapting products and technologies to local circumstances in a foreign country. This would happen if firms need to adapt their existing products to local taste, to local circumstances such as climate, or when additional peripheral products are in need in a foreign location. Similar motives may exist for other parts of the value-chain of a firm, such as marketing or production.

Because this type of $R \& D$ is specifically aimed at the foreign locale, it will under many circumstances be most efficient to undertake them in the specific foreign country or region. This has the advantage of close interaction with local people and other production factors, and to perform prototype testing under actual local circumstances. There are two essential points about this type of foreign R\&D. The first is that it is a substitute to domestic R\&D, and does not add in a radically new way to the specific technological capabilities of the firm. The second is that this type of foreign $R \& D$ does not show any particulat tendency to locate in specific foreign regions on the basis of the technological infrastructure of those regions. $\mathrm{It}$ is foreign demand that attracts this type of $R \& D$, not foreign technological capabilities.

The second type of foreign R\&D is called asset-seeking (Dunning and Narula 1995). This argument starts from the assumption that different regions are characterised by different knowledge bases, something that will be discussed below. The specific nature of the foreign technological knowledge base pulls the firms into doing foreign $R \& D$. Instead of building on its existing technological capabilities and seeking to extend these to foreign circumstances, the firm now aims at utilizing the local knowledge base to develop new capabilities that are complementary to its existing capabilities. The tapping into local knowledge bases may either be aimed at the (semi-) public research 
infrastructure, such as universities and research institutes, or at knowledge developed by other firms. The firm may use this knowiledge to expand its existing products and technologies into new technological directions, or to fuse its existing line of business with new dewelopments in certain technological fields.

This chapter does not aim to investigate whether foreign $R \& D$ is dominated by either asset-exploiting or asset-seeking $\mathrm{R} \& \mathrm{D}$, which is the dominating research question in the business literature (e.g., Patel and Vega 1999; Le Bas and Sterra 2002; Criscuolo, Narula et al. 2001). However, the distinction between the two forms of foreign $R \& D$ is important because they have different implications for the spatial dimension of knowledge. Whereas asset-exploiting foreign $R \& D$ does not lead to regional clustering of $R \& D$ activities (although it may follow a pattern of concentration of general economic activity), the asset-seeking variety is crucially linked to spatial concentration of R\&D activities. In order to see this, one must realise that the asset-seeking argument pre-assumes that knowledge bases differ between locations, and that they cannot easily be tapped into from a distance (e.g., the home base of a MNE). The asset-seeking strategy of foreign $R \& D$ would not be necessary if researchers in the home base lab of a MNE would be able to use the knowledge base of a foreign region. In other words, the asset-seeking argument assumes that geography matters.

This brings us to the second research hypothesis formulated at the beginning of this section. Continuing the line of argument, the choice for a particular region in terms of foreign R\&D location will depend on two factors: the nature of the region's local knowledge base, and the extent to which the entering firm will be able to tap into this knowledge base. These two factors are extensively covered in the literature on the spatial nature of knowledge systems (see, e.g, the paper by Morgan (2004) for an overview).

Traditionally, one may point to two factors that enhance the local concentration of certain types of knowledge building or R\&D. First, there is the traditional argument about agglomeration economies that is related to the availability of common resoutces. Examples of these common resources include a specialised workforce of skilled engineers with experience in a certain field of research, a university offering a specialised degree relevant for the type of R\&D, specialised firms that can supply certain types of instruments and/or services, or even a notion such as technological culture (Saxenian 1994). When these types of resources are important inputs into the $R \& D$ process, an emerging spatial cluster of $R \& D$ activities may provide important advantages to the "members" of such a cluster, and thus a self-reinforcing process may set in that leads to strong spatial concentration.

Hospers (2003) uses the "Blue Banana" concept to explain the concentration of innovative activity of MNEs across Europe. He essentially 
makes use of Schumpeterian economics, structural change theory and the arguments provided by the agglomeration economies to provide an explanation for the observed concentration of innovative performance in an area termed the "Blue Banana". Besides this Blue Banana area, running from London to Milan, he also distinguishes two other possible growth regions, the "Sunbelt", running from Milan to Valencia and the "Yellow Banana" from Paris via Cologne to Warsaw.

The second factor that may explain the spatial nature of knowledge is related to the nature of knowledge itself. Here the distinction between knowledge and information becomes of crucial importance. While information is by its very nature rather easy to codify, this is often not the case for knowledge (see, e.g., Cowan, David et al. 2000; Gertler 2001; Johnson, Lorenz et al. 2002 provides a discussion specifically related to geography). Knowledge, contrary to information, has a high degree of tacitness. This implies that it must be transmitted by close personal interaction as in a teacher-pupil relationship, or by a combination of codified sources, experimentation and hands-on trialand-error applications on the knowledge-receiving end.

This argument was first introduced into the literature on firms' technological capabilities and regional innovation systems by Von Hippel (1994). He used the term "sticky knowledge", where the "stickiness" of a given unit of information is defined as the incremental expenditure required to transfer that unit of information to another location where the information is useful for the information seeker (Von Hippel 1994: 430). With this term "sticky knowledge" Von Hippel (1994) indicates that knowledge cannot be transferred at non-significant costs between individuals or regions. When this cost is low, the information is considered non-sticky; on the other hand when the cost is high the "stickiness" is considered to be high too. The most important reason for knowledge to be "sticky" has to do with the tacit nature of knowledge. Even for tacit knowledge that is readily available on the marker, physical or geographical proximity is still necessary, since the transmission of tacit knowledge requires personal contact. "The marginal costs for transmitting codified knowledge does not depend on the distance over which it is transmitted, but with tacit knowledge this cost increases with distance. As a logical outcome of this, he argued that firms aiming at tapping into a knowledge base that has been developed in a certain region would locate in this region. In this way, they would be able to hire some of the engineers with experience in the field, to set up partnerships with firms in the region, and so on.

The argument is also found in the literature on the local nature of patent citations, which are often taken as an indication of a knowledge flow (e.g., Jaffe, Trajtenberg et al. 1993; Jaffe and Trajtenberg 1996; Maurseth and Verspagen 2002). The issue here is whether or not patent citations (and hence knowledge flows) between firms, or from (semi-) public knowledge institutes to firms, 
depend on geographical distance. The above quoted studies find that both in the U.S. and Europe, knowledge flows tend to be more intense between parties that are located close to each other in space.

Based essentially on a combination of this "spatial argument" and the theory of MVEs outlined above, Cantwell and lammarino (2001) have suggested that Europe is chatacterized by a division into three types of regions: higher order regions, intermediate onder regions and lower order regions. The last category is chatacterised by low technological activity, while the first two categonies have high technological activity. What distinguishes higher-order regions from intermediate order regions is the range of their activities over fields and the change of this. Intermediate order regions "attract innovative activities for a specific set of specialized expertise which can be accessed by asset-seeking large firms", whereas higher order tegions are "tmore likely to attract a broad range of both indigenous and foreign innovative activities "... large firms and [MNEs] located there will generally try to extend their established lines of specialization through intra-firm networks" (Cantwell and lammarino 2001: 1010-1011).

The short literatute review in this section suggests that there may be reasons for innovative activity (by the firms in our sample) to be clustered in a limited number of regions (if asset-seeking behaviour plays an important role), or reasons to expect that the distribution is more even (if asset-exploiting behaviour is important). We will use a descriptive approach to assess which of the two cases prevails. Given that out findings imply that regional concentration is a very relevant phenomenon indeed, we will extend our descriptive approach to investigate the relevance of some of the causal mechanisms discussed above (localised nature of knowledge flows).

We will follow the literature by using patents as a source of information. However, contrary to, for example, Cantwell and Iammatino, we will use European patents. This may be considered as an important complement to the existing analyses based on US data, because the European patent system may be more relevant for Eutopean based activities. We will not, like Cantwell and Tammarino, focus the analysis on a small set of predetermined tegions, but instead take a broad view including 125 regions in the former 15 European Union countries plus Switzerland and Norway.

\subsection{Data}

This chapter follows in a tradition that uses patents as an indicator of technological activity. As has been noted before (see chapter 2), this indicator is far from perfect. Some of the most well-known problems are that not all innovations are patented, not all patents are commercialised, that patents may 
vary wildly with regard to innovative size, and that the so-called propensity to patent (percentage of all inventions that is patented) varies by industry. Nevertheless, most authors surveying these issues tend to conclude that patent statistics can be useful indicators. For example, as a conclusion of an analysis comparing innovation count data and patent data as indicators of innovation at the regional level for the USA, Acs, Anselin and Varga (2002: 1080) conclude that theit "empitical evidence suggests that patents provide a fairly reliable measure of innovative activity".

Our data source is the European Patent Office (EPO) database on patent applications. We select all patent applications ${ }^{4}$ with a priority date in the yeats 1994-1997 (inclusive), whether they are granted, have been rejected (or withdrawn), or are still under review. Our sample of firms is limited to large multinational firms that appeared on the Fortune 500 list in 1997, supplemented by a few large firms from the Fortune lists in earlier years. Of these, we selected a sub sample of firms active in high- to medium-tech sectors. For these firms, we made use of the Dun \& Bradstreet Linkages database to construct a list of their subsidiaries. The Dun \& Bradstreet Linkages database includes only full, i.e., one hundred percent, subsidiaties. We tefer to this list as the "group". The version of the Dun \& Bradstreet Linkages database we used is from late 1998, and represents thus the parent-subsidiary relationships at, or in fact slightly before that point in time. Of course these connections have not always been like they were in 1998 . This is the reason why we will use only a limited set of years for our patent database. When analysing patent counts per firm, we use 1997 as the best approximate year, and we can be fairly confident that our data are correct in the large majority of cases. When it comes to analysing patent citations, we need to take into account a longer period, because the dates of cited and citing patents usually lie apart several years. We use the period $1994-1997$ in this case.

A total of 171 firms were investigated in this way. From this large sample, only firms with a minimum of 25 patents during 1994-1997 were included in the analysis (sce below for a note on how patents were counted). The resulting dataset comprises 87 firms, divided over the following sectors (numbers in brackets are the number of firms in the analysis): Aerospace (5); Chemicals (13); Computers (5); Electronics (13); Industrial and farm equipment (6); Petroleum (8); Pharmaceuticals (9); Metals (4); Motor vehicles (13); Scientific, photo and control equipment (3); Semiconductors (1), and Telecommunications (6). In the parts of the analysis below that refer to sectors, we will usually regroup the sectors into three large groups (electronics: computers, semiconductors, electronics, telecommunications; chemicals: pharmaceuticals, chemicals,

\footnotetext{
4 We will use the term "patents" loosely, i.e., also when we refer to patent applications.
} 
petroleum; other, aerospace, industrial and farm equipment, metals, motor vehicles, scientific, photo and control equipment).

In order to capture the geographical dimension of the data, we specify 125 European regions, largely based on the Nomenclature of Territorial Units for Statistics (NUTS) regional classification applied by Eurostat. We use essertially the same regional breakdown as in Maurseth and Verspagen (2002), which includes both NUTS 1- and 2-digit regions, but add a few countries. The countries in the database now include Norway, Sweden, Finland, Denmark, Ireland, the United Kingdom, the Netherlands, Germany, Belgium, Luxembourg, France, Italy, Spain, Portugal, Greece, Switzerland and Austria. In the case of Germany and Austria, small urban regions (Bremen, Hamburg, and Vienna) were merged with neighbouring or surrounding regions to awoid too small geographical entities. For Ireland, Finland and Luxembourg, we do not have a regional breakdown and these countries are included as a single region. For Switzerland, Norway and Denmark, the classification used was not based on NUTS, but instead on national administrative definitions (in Switzerland it was based on Cantons, in Norway on Fylken, in Denmatk on Landsdelen.

The assignment of patents to regions is done by using the postal code of the inventor address. The exact procedure used to do this is similar to Caniels (1999). We select patents by their applicants (i.e., the multinational firms groups), but assign patents to geographical regions by their inventors. By nature of the research question, we are interested in the geographical location of the inventor rather than the applicant, because this corresponds closer to where the actual research that led to the patent was unciertaken. In order to cope with multiple inventors and/or applicants of a single patent, we apply a fractional counting method. Suppose a patent has $n$ inventors and $m$ applicants ( $n$ is usually latger than $1, m$ usually equal to one). Suppose that of the $n$ inventors $n_{i}$ (smaller or equal to $n$ ) are located in region $A$, and that $m$ (smaller or equal to $m$ ) of the $m$ applicants are subsicliaries of firm B. Then a fraction $1 /\left(H_{a} m_{B}\right)$ of the patent is assigned to location $\mathrm{A}$ of firm B.

\subsection{The spread of patenting over European regions}

As was already noted above, it has been argued that $R \& D$ is an important case of "non-globalisation" (Patel and Pavitt 1991). Table 5.1 shows that argument is valid only for a relatively small part of our sample of multinational fitms. The table gives the share of patents originating from foreign regions in total patents per firm, with prionity date in $1997.5^{5}$ The table includes only

\footnotetext{
5. I.e, the able gives the mean, median and standard deviation over $i$ of the variable $x_{i}=F_{i} / T_{i s}$ whete $\mathrm{i}$ indicates a firm, $F_{\mathrm{i}}$ is parents of the firm inwented in a foreign location, and $T_{\mathrm{i}}$ is total patents of the firm.
} 
European firms, since we do not have data for patenting in locations other than European regions (i.e., the home base of the Japanese and US firms in the sample). The numbers given refer to all tirms in the given sector, or the total sample.

Table 5.1. Share of patents invented in foreign regions in Europe by European based MNEs, 1997

\begin{tabular}{|c|c|c|c|c|}
\hline sector 7 sample & Mirnoter of & Mean & Median & $\begin{array}{l}\text { Siandard } \\
\text { deviations }\end{array}$ \\
\hline Total sample & 52 & 018 & 0.11 & 021 \\
\hline $\begin{array}{l}\text { Chenicals, Pharnaiceutrals } \\
\text { perroleum }\end{array}$ & & 118 & 0.13 & 1013 \\
\hline $\begin{array}{l}\text { Computers, electronics, } \\
\text { telecomm. }\end{array}$ & 12 & 0.24 & 0.15 & 0.27 \\
\hline Allother sectors & 19 & 0.5 & 00 & 0.24 \\
\hline
\end{tabular}

Source: own calculations on the basis of EPO data. Other sectors include: acrospace, industrial and farm equipment, basic metals, motor vehicles, scientific, photo and control equipment.

The mean of the shate of patents originating from foreign locations differs between the sectors in the database: it varies from 0.15 to 0.24 . The highest values are found for the British company BTR $(100 \%)$, the Swedish company Electrolux (81\%), the Swiss company $\mathrm{ABB}(61 \%)^{6}$, and the French company Alcatel (52\%). These are the only companies with more than half of their patenting activity abroad. The median of the share of foreign patents is in all cases smaller than the mean, which indicates that the distribution is skewed towatds the left side, i.e., towards firms with low values for the internationalisation of $\mathrm{R} \& \mathrm{D}$. But the median value is clearly above $10 \%$ for the two largest sectors in our database, i.e., chemicals and electronics (but clearly lower for the other sectors in the database). In addition, it has to be born in mind that this number would increase if one takes into account patents in the US or other non-European parts of the world. Finally, the standard deviation is rather large for all sectors, indicating that there is indeed a large variety berween firms with regard to their level of R\&D globalisation. The findings in Table 5.1 support the case that foreign $R \& D$ is a substantial part of all R\&D activities by the firms in our sample.

\footnotetext{
$6 \mathrm{ABB}$ is one of the companies for which it is hard to determine the home base. We have used Swizerland, while Sweden would have been the other candidate country. There are two other companies for which this is difficult: Unilever and Royal Dutch Shell. We decided to use the Netherlands as the home base for these counrries (the UK was the other choice awalable).
} 


\section{CHAPTER V}
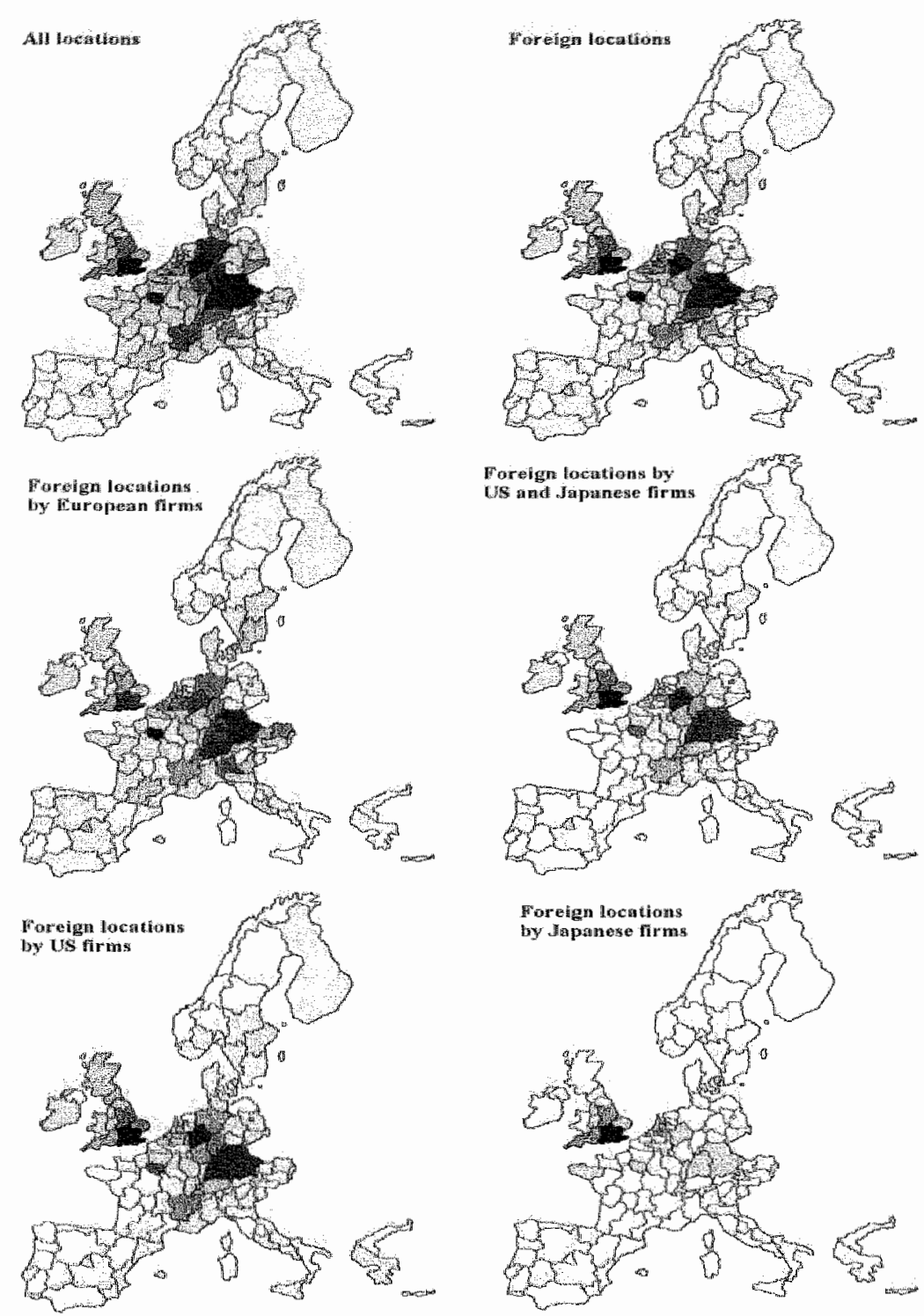

Figure 5.1. The presence of large MNEs R\&D activities in European regions dight shades indicate a low anount of firms, dart'k shades a high amount of firms)

In order to provide an overview of which European regions attract most R\&D activity by the firms in our sample, we constructed the maps in Figure 5.1. The shading of the maps is an indication for how many firms have a positive number of patents from that particular region. Light shades indicate 
low activity (lightest shade is zero firms), while dark shades indicate the high activity (the maximum varies per map). The map in the upper-left comer refers to the total of 87 firms in the sample. What is clear is that the central part of the map is most in demand in terms of locations for R\&D. Three German regions, the Southeast of the UK (London) and the region around Patis are on top of the list. These regions all attract around 45 firms, i.e., slightly more than half of all the firms in the sample are active in these regions. Other regions in the centre of the map also rank telatively high, including most other tegions in West Germany, South of the Netherlands, North-West of Italy, and Southeast of France. Outside the cluster of regions in the centre, only some isolated regions attract a significant amount of firms: the regions around Stockholm, Madrid, Rome, as well as Scotland and Ireland. In comparison with the list of higher order regions defined by Cantwell and Tammarino (2001), we find that their regions are included in the top of our list as well. However, our results clearly show that in terms of the sheer numbers of firms active in regions, the ctiterion of having only one region per country is rather restrictive.

The pattern changes only slightly if we leave out the R\&D activities of the European firms in their home country. This is displayed in the map in the rightupper comer. The most prominent difference with regard to the previous map is that the three top German regions are now somewhat less pronounced, although still quite high up on the list. Now the regions around London and Paris are leading the ranking (35-40 firms). We also find that a number of higher order regions of Cantwell and Iammarino are no longer very prominent when only foreign activities are considered. This is the case for Stockholm, and, to a somewhat lesser extent, the area around Brussels.

The next four maps refer to a subset of the second map, i.e., they each single out a specific category of foreign activities. The third map (centre-left) displays all foreign activities by European firms. What is notable here is that the region atound Paris is much favoured as compared to the other top locations from the previous maps. The picture is quite opposite with regard to the Japanese and US firms taken together. This is in the centre-right picture. Here the Southeast of the UK (London) and, to a lesser extent, Germany stand out. What is notable also is the almost complete absence of Japanese and US firms from other parts of Europe than Central Europe. Among the non-European firms, the US firms are a larger group than the Japanese firms. The last two maps single out the data for these two groups separately. The US firms are in the lower-left comer. Here we see some activity in most of the central regions that were seen before. However, London and the German regions particularly attract the US firms. The few Japanese firms that are active in Europe (rightbottom corner) are mostly concentrated in the area around London.

Concluding, we observe a tendency for R\&D activities by MNEs to be concentrated in relatively few regions in Europe. Large parts of Europe do not 
see any, or wery lintle R\&D activities by the firms in our sample. Moreover, foreign R\&D activities tend to be aimed at an even smaller set of regions, with US and Japanese firms being again somewhat more selective that European firms doing R\&D in foreign European countries.

Given the rather concentrated pattern of MNE presence in European regions, it becomes of interest to look at the spread of patenting over regions at the level of individual firms. One indicator that can be used for this is the Herfindahl-equivalent-number-of-regions indicator:

$$
Q_{i}=1 / \sum_{j}\left(P_{i j} / \sum_{k} P_{i k}\right)^{2},
$$

where $Q_{i}$ is our indicator for firm $i$ (charted on the vertical axis of Figure 5.2), $P_{i j}$ is the number of patents of firm $i$ originating from region $j . Q$ can be interpreted as the number of regions that would generate the same value of the indicator, but with equal shares of patenting in all regions. The larger (smaller) this value is, the more (less) spread out are the $R \& D$ activities of the firm.

The indicator is displayed in Figure 5.2, against the logarithm of the total number of patents for each firm in Europe for the period 1994-1997. The two axes drawn in the figure correspond to the median values of the indicators. There is no clear relationship between the two indicators in the graph. High values of the spread over regions are found for intermediate values of total patenting rather than the extremes of this distribution. There are also no clear differences between the three sectors in the graph.

The minimum for the spread variable lies slightly above one, which would correspond to the case where almost all patenting of a firm is concentrated in a single region. The highest value for this indicator is reached at a value just under 11 (for the Swedish company Electrolux, the next highest value is the US company Du Pont), while the median is at 3.2. The overall picture is thus one in which most firms have significant foreign patenting activities, but these are concentrated in a limited number of regions, both from a European spatial one, and from the point of view of the number of locations per firm. There are also some firms, however, which patent from a large number of regions and source a large share of their total patents from foreign (European) countries. 


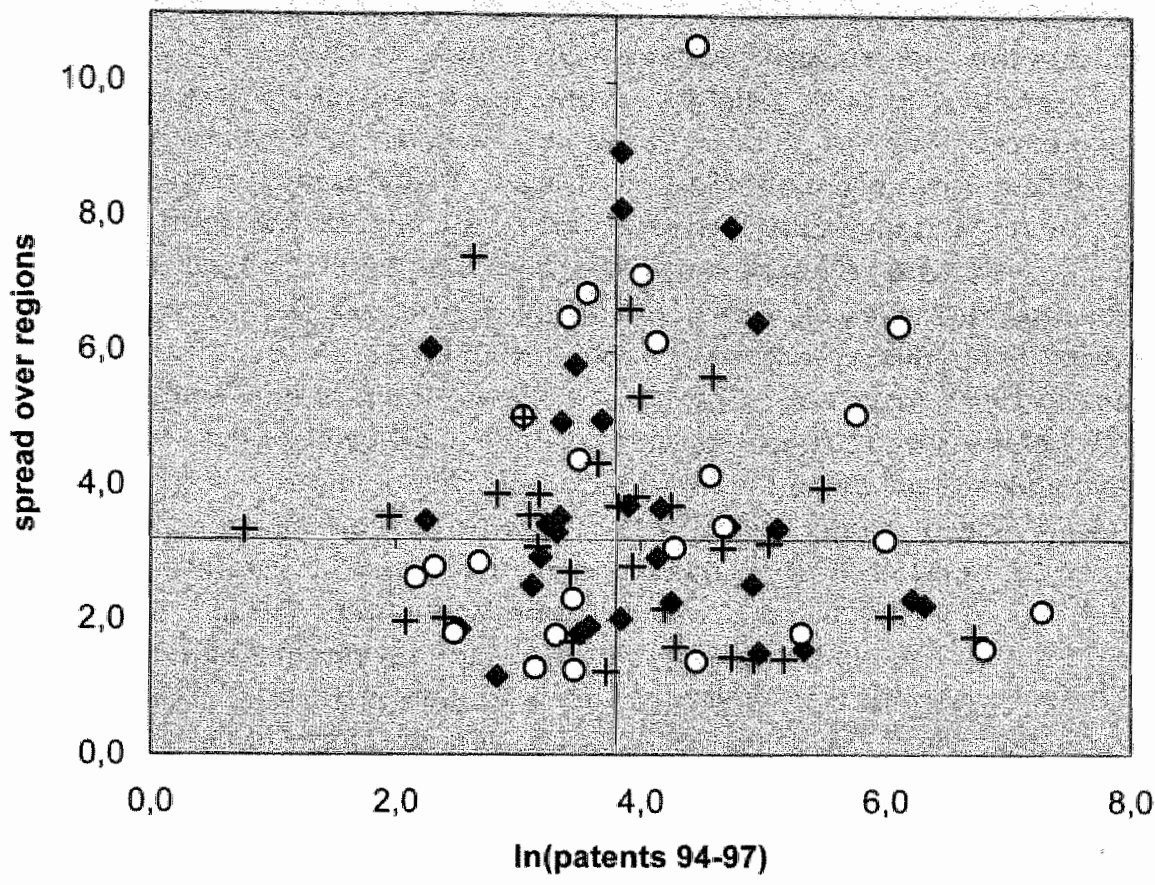

chemicals etc. O electronics etc. +others

Figure 5.2. The mumber of patents vs. the average spread of patenting over European regions, firm level.

\subsection{The Blue Banana and beyond}

So far we have looked at the distribution of patenting activity across the European regions without looking deeper into the underlying reasons for this distribution. We now want to provide some explanation for the observed phenomenon and will try to relate it to other researches. We found that even though modern-day communication systems would seen to eliminate geography as an important factor in economic life, this is apparently not the case for innovations. We found a clear indication for the concentration of economic activity, and especially innowation activity, in geographical space for the firms in our dataset. Innovation rutns out to be even more concentrated in space than the production of goods and services (Paci and Usai 2000, 2001). 
For innovative performance of company's, geography apparently still matters. This notion of the geographical concentration of economic activity is not new. Alfred Marshall (1936) already acknowledged this phenomenon. He recorded three essential advantages that would lead firms to cluster. First there is the collective market for skilled workers who posses industry-specific competencies. Second there are the non-tradable and intermediate inputs prowided by local suppliers. And third, there is the easy transmission of new ideas which leads to increased productivity via improvements in production, organisation and in technical features. These three factors will enhance a sort of "industrial atmosphere", which is able to endorse economic growth. Other scholars have pointed to the path-dependency of "economic geography" (e.g. Cantwell 1991; Krugman 1991). According to Cantwell and Iammarino (2003: 5) "Technological change is a path-dependent process in the sense that the probabilities of adopting a certain kind of technology are influenced by past decisions, which in turn constrain the range of existing choices. Therefore, the industrial composition of innovative activities in a given location reflects past technological accumulation ". Also Nelson and Winter (1982) emphasise the importance of location for firms seeking to tap into the innovation systems of other firms. Again they point to the path-dependency of the innovation process, but also to the firm-specific nature of such innovations. This brings us to a third argument about geographical concentration and deals with the distinction between information and knowledge, and especially the transmission of both. While information is free in principle and relatively costless and easy to transport this is not the case for knowledge. This has to do with the tacit nature of knowledge on the one hand and the absorptive capaciry necessary to interpret and absorb outside knowledge on the other hand (Nelson and Winter 1982; Von Hippel 1994). The cost of transmitting information is more or less indifferent to the distance over which it is transported; the cost of transmitting knowledge is not (Von Hippel 1994). Von Hippel (1994) uses the term "sticky knowledge" to refer to the ease with which a given unit of information is transported to another location. "The "stickier" the given unit of information is, the higher are the incremental costs to transport this information to another geographical location. The costs also increase with the distance over which it is transported. Since the transmission of tacit knowledge requires close interpersonal contact, even for tacit knowledge that would be freely available on the market, close geographical proximity is a prerequisite. From this point of view it would make sense for firms to cluster in a certain region, for this would enhance knowledge transfer between the firms. This clustering of firms in turn makes the designated area even more attractive for other firms. In addition these areas usually have fully-evolved technological markets, a well-developed local science and technology infrastructure, institutional relationships, services and various facilities (Cantwell and Iammarino 2003). The path-dependency 
and firm-specificity of the innovative process determine the costs of articulating new knowledge. These costs in turn will determine the degree to which tacit knowledge will stay tacit over time. Geographical clustering can reduce these costs (Cantwell and Iammarino 2003) stressing the fact that knowledge spillovers are basically geographically bounded (Jaffe, Trajtenberg et al. 1993; Almeida and Kogut 1997).

For Europe this concentration of economic activity in only certain geographical locarions is sometimes named the "Blue Banana" after the shape of the area on the map of Europe and the coincidental colour of the area when it was first introduced (Hospers 2003). Even though Europe is being unified ever more we still can detect a more or less homogenous economic area where we can find the greatest growth potential in Europe. The area runs mote or less from London via the Benelux and the Rhine area towards Milan (see figure 5.3)

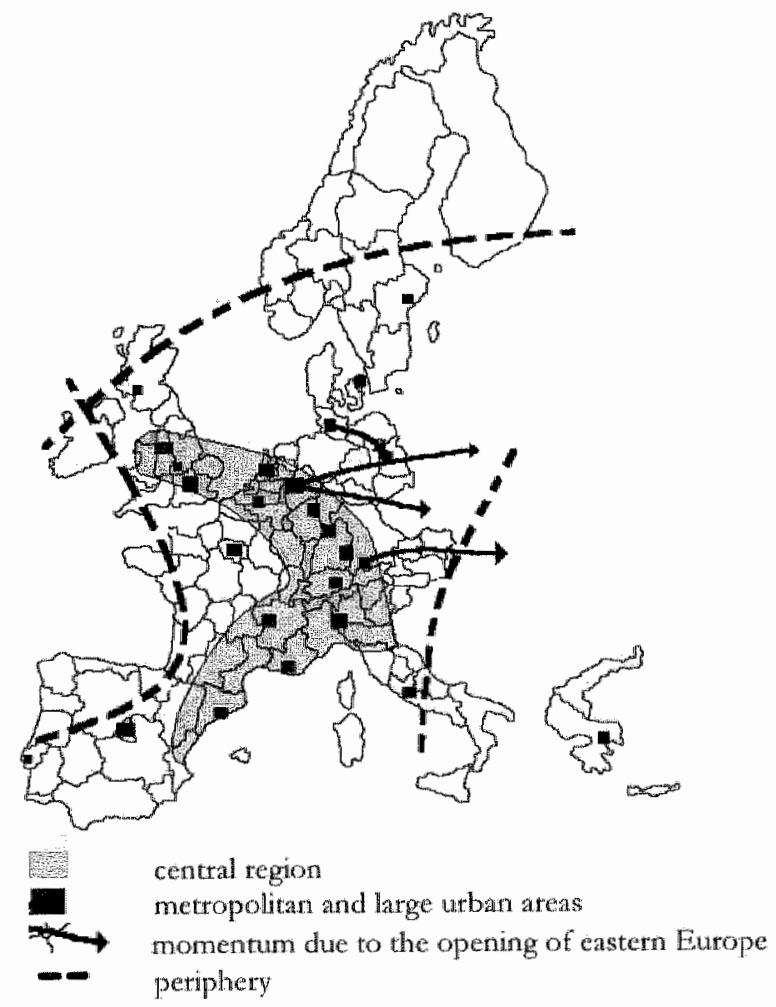

Figure 5.3: The Blue Banana and Begond

Source: Reproduced from L.Schäel (ed): Wirtschaftsgeographic de Europäischen Gemeinschaft, Stuttgart 1993; Uni-TB. 
The Blue Banana area differs from other European areas in demographic, economic, infrastructural and cultural-educational aspects (Hospers 2003). The area is highly populated and highly urbanised, comprising many large and medium sized European cities (e.g. London, Amsterdam, Brussels, Basle and Milan). More than $40 \%$ of the EU population lives in this area, which is also described the "city belt". "... [T] he regions within the Blue Banana have higher per capita incomes and lower employment rates than the rest of Europe. Besides, this zone contains large industrial concentrations $[. .$.$] as well as$ strongly developed services, banking and public administration. [...] the Blue Banana has a well-developed physical and telecommunications infrastructure as well as dense traffic networks. Finally this area [has a] relatively large supply of cultural and educational facilities" (Hospers 2003: 78). "The Blue Banana area probably dates back to medieval or even Roman times and might reflect the old trading routes via the Alps and along the Rhine. It was also this area where the Industrial Revolution spread over the whole of Europe. Apparently the longterm structures continue to be important even until today (Hospers 2003). Recently two orher growth zones are being recognised in Europe: the Sunbelt running broadly from Milan to Valencia along the Mediterranean coast; and the Yellow banana running from Paris via Cologne and Berlin to Warsaw (Hospers 2003).

If we look back at our own results (see figure 5.4) we can see that we also found that the areas that lie in the Blue Banana region show clearly a higher innovation activity. We do not find conclusive evidence for the supposed Sunbelt or the Yellow Banana region, but we need to note that our data consists only of data for the former $15 \mathrm{EU}$ countries supplemented with Switzerland and Norway. For a clear view on the Yellow Banana region this might not be enough. We observed however higher patenting activity for the area around Paris and for some northern German regions, which could be part of this Yellow Banana region. Also in the south of France we found higher patenting activities, which could be part of the Sunbelt region. For Spain however only the area around Madrid has high patenting activity, the northeast of Spain shows only very low patenting activity.

As reported before Cantwell and Iammarino (2001) make the distinction between higher-order, intermediate and low-order regions. Their research provides support for the proposition that a geogtaphical hierarchy of regional locations exists across national boundaries within the $\mathbb{E U}$. Besides the loworder regions characterised by low innovative activity, the core European regions can be divided into two groups, the intermediate region with traditional specialisation for the region, and the higher-order region with developments in fields of high technological opportunities. Intermediate-order regions like for instance the Northwest in the UK and Piemonte in Italy attract innovative activities for a specific set of specialised expertise, which outside firms are able 


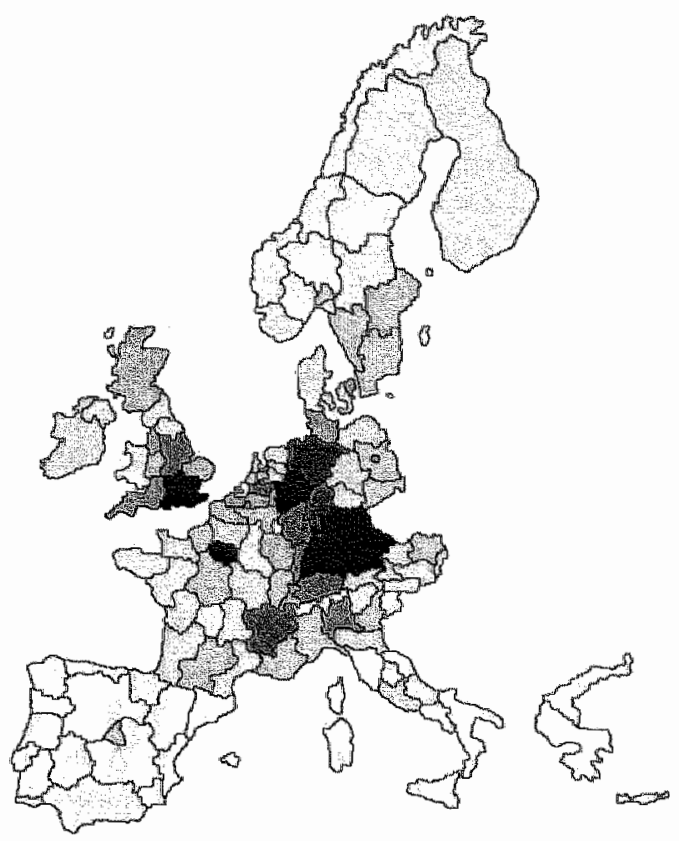

Figure 5.4: Patenting activity by all firms in our dataset (light shades indicate low patenting activity, dark shades high patenting activity).

Source: own calculations based on our own datenset.

to tap into in order to increase their capabilities in this particular field of expertise (Cantwell and Iammarino 2001). Higher-order regions on the other hand are characterised as being overall highly dynamic in the senge of technological and productive activities, overall infrastructure, financial facilities, business climate and business culture, and the openness to external netwotks. Higher-order regions are thus able to compete on an international, cross-border scale (Cantwell and Iammarino 2001). Following the rationalisation process of firm innovative processes Cantwell and Iammarino (2001) expect more and more competition especially among the higher-order regions in Europe. For our result this would mean that MNC innovative activity will get more and more concentrated in the area described as the Blue Banana. In this area we find the higher-order regions of Europe with their highly developed transportation, financial, and educational infrastructure. MNCs that want to be on the cutting edge of innovative activity have to be present in these regions, thereby reinforcing the importance of the regions. For intermediate-order 


\section{CHAPTER V}

regions it will be difficult to become a higher-order region, where being close to 3 higher-order region might be helpful in succeeding. For low-order regions it will be virtually impossible to change into a higher-order region. It would requite substantial investments in the region and possibly also in changing the mind setting of the managers.

Hospers (2003) makes, based on the "three sector hypothesis" by Fourastié (1951), more or less the same division among regions as Cantwell and Iammarino (2001) do. He names the regions the peripheral agricultural areas, the intermediate industrial areas and the core service areas. According to Hospers (2003) the core service areas are, most likely, the new growth areas of Europe because of theit diversified economic and institutional structure and advanced educational and infrastructure facilities" (Hospers 2003: 83). Flexilbility is necessaty in order to absotb new techno economic developments and to develop "new combinations" (Hospers 2003). The intermediate industrial areas might also dexelop into new growth areas, but just like for the intermediate-order regions of Cantwell and Iammarino (2001) this will be difficult for them. The peripheral agricultural areas will experience even more difficulty to change since they lack the change the intermediate industrial areas already went through and have to change immediately to a core service area. According to F-lospers (2003) what is most important when concluding where the European areas are going to is to look at where they ate coming from. Therefore Hospers (2003) expects the Blue Banana area to stay the most important growth atea in Europe for at least the coming decades.

Apparently the division of patenting over areas that we found based on our own data is rather stable over time, and is thus not changing quickly. And if changes occur they will very probably be gradual and needing huge investments in for instance infrastructure and the educational system. Also we found that the Bluc Banana area is indeed the most important innovative atea in Europe, supplemented with a few peripheral regions. Due to the huge investments necessary in order to come to a patentable new invention we can expect that not many changes will occut in the division of patenting across the European regions in the near future. Patenting will very probably be concentrated in the areas we now observed as being highly innovative for the near future and even beyond.

\subsection{Geographical distance and patent citations in Europe}

So far, it has been shown that the R\&D activities of MNEs in Europe are indeed concentrated in a limited number of regions. Attention has been paid to the mechanisms that may lead to this tendency. This section will investigate the issue of sticky knowledge flows in a more detailed way. In order to do this, we 
need to operationalise two additional dimensions in the database: distance and knowledge flows.

The starting point for measuring the distance between regions is the classification in terms of NUTS units introduced above. The distance between two regions $p$ from $q$ is measured by counting the (minimum) number of botders on the NUTS map one has to cross to reach region $p$ from $q$. For the sake of this calculation, some regions with sea areas between them have been defined as actual neighbours, in order to make all regions reachable from all other regions. This is an admittedly naïve way of measuring distance, which could be improved in a number of ways, such as measuring actual distance in kilometres or miles, or by measuring virtual distance in terms of travelling time. However, the analysis in Maurseth and Verspagen (2002) showed that for a subset of regions in the present sample, the results do not differ substantially between the present distance measure and a more sophisticated one based on actual distance in kilometres.

In order to measure knowledge flows, we will use patent citations, i.e., references to previous patents given in patent documents. The legal purpose of patent citations is to indicate which parts of the described knowledge are claimed in the patent, and which parts have been claimed earlier by other patents. From an economic point of view, however, the assumption is that a reference to a previous patent indicates that the knowledge in the latter patent was in some way useful for developing the new knowledge described in the citing patent. This is the line of reasoning offered in the studies by e.g., Jaffe, Trajtenberg and Henderson (1993), Jaffe and Trajtenberg (1996 and 1998) and Maurseth and Verspagen (2002). The detailed case study by Jaffe, Fogarty and Banks (1998) on a limited sample of patents, as well as Jaffe, Trajtenberg and Fogarty $(2000 \mathrm{~b})$ conclude that patent citations are a "valid but noisy measure of knowledge spillowers". ${ }^{7}$

It should be emphasized that knowledge flows are a much broadex concept than what is captured by patent citations (U.S. or European). First, in order for patent citations to take place, both the knowledge receiving and knowledge-generating firm must be actively engaged in R\&D and apply for (European) patents. Patents citations are knowledge flows between researchers, not, for example, between supplier and customers. Second, patents are an ultimate example of codified knowledge, because they require an exact description of technological findings according to legally defined methocks. Thus, one can have little hope of identifying tacit knowledge flows by means of the paper trails that patent citations leave. One may assume, however, that the codified knowledge flows of patent citations go hand-in-hand with more tacit

\footnotetext{
"Maurseth and Verspagen (2002) discuss the differences between the U.S. and European pattent system that are relevant for the interpretation of parent citations.
} 
aspects of knowledge flows, but this argument remains admittedly specularive (Patel and Pavitt 1997). The conclusion is therefore that our analysis will only refer to a very specific and limited form of knowledge generacion activities and knowledge flows, and our data have important imperfections. The approach has, however, the advantage that we can make use of a very detailed and precise clatabase.

Patent citations involve a time lag. Just after the patent has been applied for, only little citations to it will appear. The number of citations rises until it reaches a peak after (for the case of EPO patents) two or three years. Then the number of citations gradually falls to zero over a prolonged period that may take up to 20 years. In order to stay as close as possible to the year (1997) for which our parent-subsidiary relationships for furms hold, we looked at cited patents that have priority date in 1994, and citing patents in the years 1994 1997. Although the 3-year citation lag that this implies is indeed rather short, it does capture a significant amount of a typical patent's citation life.

Because a patent citation involves two patents, the way of counting changes slightly as compared to just patent counts. In principal, the fractional way of counting is maintained, but we will look only at whether the number of citations between two units is positive or zero. As a unit that may cite or be cited, we will take the location of a firm in a region. Thus, if firm $A$ has patenting from regions $i$ and $j$, and firm $\mathrm{B}$ has patenting from regions $p$ and $q$, all possible citation links include $\mathrm{A} i-\mathrm{A} i, \mathrm{~A} i-\mathrm{A} j, \mathrm{~A} i \mathrm{~B} p, \mathrm{~A} i-\mathrm{B} q, \mathrm{~A} j-\mathrm{A} i, \mathrm{~A} j-\mathrm{A} j, \mathrm{~A} j-\mathrm{B} p$, $\mathrm{A} j \mathrm{~B} q, \mathrm{~B} p-\mathrm{A} i, \mathrm{~B} p-\mathrm{A} j, \mathrm{~B} p-\mathrm{B} p, \mathrm{~B} p-\mathrm{B} q, \mathrm{~B} q-\mathrm{A} i, \mathrm{~B} q-\mathrm{A} j, \mathrm{~B} q-\mathrm{B} p, \mathrm{~B} q-\mathrm{B} q$. Note that because citations are directional (it matters who cites and who is cited), $A i-A j$ is different from $\mathrm{A} j$-A $i$. Note also that we include citations between members of the same MNE group, both if they are located in the same region (e.g., $A i-A z$ ) and if they are located in different regions (e.g., Ai-Ay). An additional dimension is added by time. If firm $A$ has patenting from region $i$ in 1994, and firm $B$ has patenting from region $p$ in 1994 and 1997, the possible citation links include $A i(94)-A i(94), A i(94)-B p(94), A i(94)-B p(97), B p(94)-A i(94), B p(94)-B p(94)$ and $\mathrm{B} p(94)-\mathrm{B} p(97)$.

Our approach will be to identify all combinations of firms/regions/years that may cite each other, simply by enumerating them as in the above, simplified examples. This is important, because the evidence presented above suggests that we cannot pre-suppose that patenting activity is randomly distributed over space. If it were, we could simply compare the spatial distance between firms/regions with positive citations to the overall mean distance on our European maps. Now that it turns out that the regions that are heavily involved in patenting ase a non-tandom selection from the complete sample of regions, we have to take into account the underlying distribution of patenting over regions. This can be done by looking at which of the potential citarion 
links are actually realised (1.e., lead to pasitive citations), and see whether or not these links are characterised by relative closeness on the map.

Note that this approach does not take into account in any special way the existing regional specialization pattern with regard to patenting. One may expect that R\&D activities of a similar kind are geographically concentrated. This could imply, for example, that there is a tendency for patents within a single technology class to concentrate in a limited space. If there were also a tendency for the citation rate between patents within the same technology class to be higher than between different technology classes, this would imply spatial concentration of patent citations. This is a tendency that would certainly be picked up by our indicator.

It is out explicit choice not to "correct" our indicator for this tendency. "The reason for this is that we would argue theoretically that the specialisation pattern of regions with regard to technology classes would at least partly be caused by the localised nature of knowledge flows. In this case, the very effect that we would attempt to fillter out of the indicator would in fact be related in a systematic way to the phenomenon that we want to illustrate. Hence we leave an analysis of the causal structure between concentration of innovative activities and the existence of localised knowledge flows to future research (we expect econometric methods might yield some insight into this issue). Of course this implies that we cannot draw any conclusions on causality between these two phenomena.

The first notable finding on citations is that the number of positive citation links is small relative to the potential number of such citation links. This is documented in Table 5.2. The number of positive citation links as a percentage of the potential number of citation links (positive links plus zero links) is always small, never exceeding $3 \%$. It is higher for within group citations, indicating that knowledge flows relatively more often between units that are part of the same MNE group than between units that are part of different groups. In fact, the fraction of between group citation links that is positive never exceeds $0.2 \%$, whereas it only falls below $1 \%$ for two of the cases in the table (computers and telecommunications) for within firm citations. We thus conclude that knowledge flows between the MNEs in our sample as indicated by patent citations are a relatively rare phenomenon.

There are also important differences between sectors. At the highest level of aggregation, i.e, all chemicals related sectors against all electronics \& telecommunications related sectors, there is a striking difference with regard to within group and between group citations. The latter type of citations is relatively low in the chemicals related sectors, while the former is relatively high. This indicates that the knowledge in chemically related patents builds to a relatively large extent on the firm's own knowledge base. 'This finding can be 
interpreted in the light of the literature on "technology regimes" (Pavitt 1984; Breschi, Malerba et al. 2000; Marsili 2001). The argument here is that different

Table 5.2. The number of citation links with positive citatons, within and between MNE groups; by sector, cited patents in 1994, citing patents $1994-1997$

\begin{tabular}{|c|c|c|c|c|}
\hline Sector & $\begin{array}{l}\text { Betweengr } \\
\text { wiling groups } \\
\text { cirations }\end{array}$ & $\begin{array}{l}\text { Postrive } \\
\text { citations }\end{array}$ & $\begin{array}{l}\text { \# zero } \\
\text { citntions }\end{array}$ & $\%$ positive \\
\hline Chemicals, phatma, petroleum & Between & 274 & 766473 & 0,04 \\
\hline he turals, platina, pe & Whing & 679 & 30910 & 2.15 \\
\hline Chenncals & Between & 113 & 217661 & 0.05 \\
\hline Chemucals & Whin & 429 & 1908 & 220 \\
\hline maceuticals & Berween & 21 & 43247 & 0.05 \\
\hline als & Whine & 180 & 6692 & 202 \\
\hline Petroleum & Benween & 14 & 28016 & 0,05 \\
\hline Peiroleun & Whinin & 70 & 5135 & 134 \\
\hline Wlectrones, computers, & Between & 397 & 401768 & 0.10 \\
\hline Heotronics, computers relecom & Wuin & 299 & 26405 & M12 \\
\hline Mlecronics & Between & 187 & 136493 & 0.14 \\
\hline Mectonds & Mutur & 218 & 16282 & 132 \\
\hline Computers & Between & 2 & 5088 & 0.04 \\
\hline Computers & Wrin & 11 & 2501 & 0,4 \\
\hline Telecommuntcations & Between & 29 & 17099 & 0.17 \\
\hline Teleconmunicatons & Whin & 64 & 7228 & 0.88 \\
\hline Antomobiles & Between & 56 & 81423 & 0.07 \\
\hline Anomolites & Whin & 106 & 8865 & 118 \\
\hline
\end{tabular}

Source: own calculations based on EPO data. Some sectors not documented due to the small amount of positive citations

sectors rely on different technology bases, and these may differ with regard to the degree of cumulativeness and relative openness to "outsiders". The chemicals knowledge base is usually cited as an example of a knowledge base that is strongly cumulative and not very open to non-insiders. The results here seem to confirm this finding. The electronics knowledge base, however, is more open and less cumulative, as indicated by the findings in Table 5.2 . 
With regard to differences within the broadly aggregated sectors, it can be noted that the three chemicals sectors are relatively homogenous, with only small differences between them in terms of the percentage of positive citation links. This is not the case for the electronics related sectors. This seems to indicate that within the broad electronics and telecommunications aggregate, there are large differences with regard to the knowledge bases underlying the individual sub-sectors, while for the chemicals related sectors, these differences are much smaller.

Electronics, computers, telecom

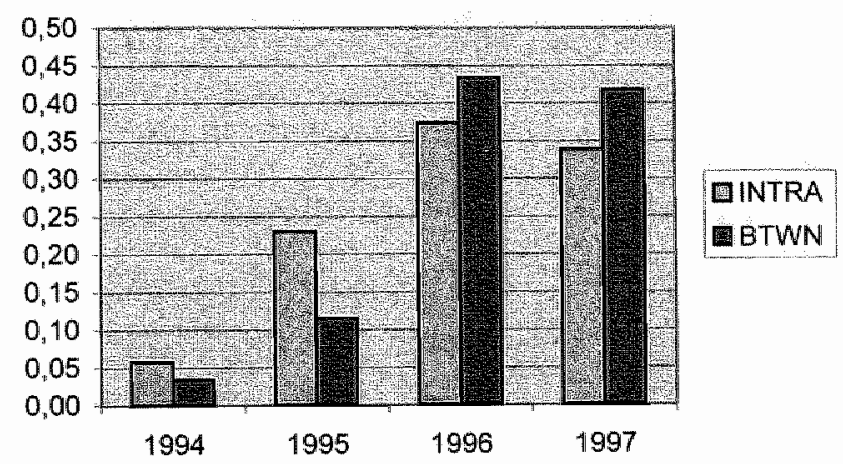

Chemicals, pharma ${ }_{y}$ petrolleum

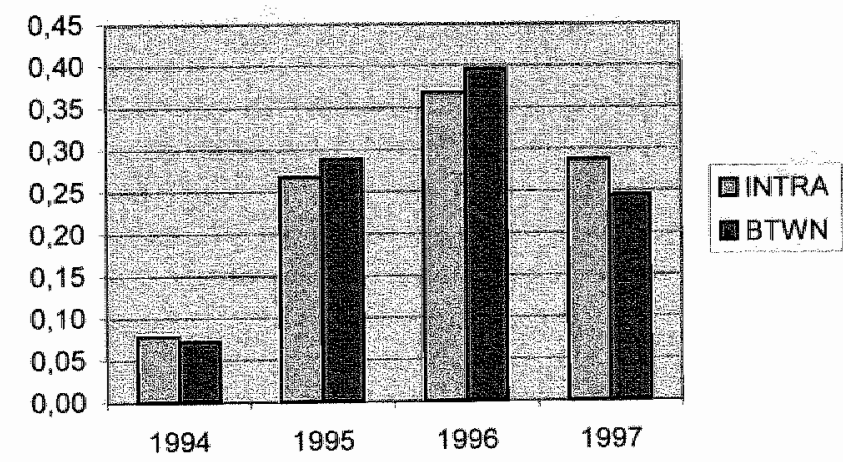

Figure 5.5. Citation pattems by sector and for within and between MNEs citations 
We now turn to a more detailed analysis of citation patterns over time. For the two broad sectors that have been defined (chemicals etc. and electronics etc.), there are enough positive citation links to make a comparison over time between within and between group citations. This is done in Figure 5.5. The bars in the figure show the distribution (fraction) of all positive citation pairs over the years $1994-1997$, i.e., within each figure, bars of the same shading add up to one. The left bar for each year is for within group citations only, the right one for between groups citations.

The influence of time on the number of citations is similar to the stylised pattern discussed above. All series have a peak in 1996, i.e., 2 years after the cited patent was filed. The number of citations occurring in the same years as the cited patent (1994) is quite small, typically around $5 \%$. The rate at which the number of citations rises from 1994 onwards differs by sector. For the chemicals sectors, there is not a very large difference between within and between group citations. In electronics, between groups citations significantly lag behind within group citation. Thus, we seem to have evidence that knowledge diffuses more rapidly within firms than between firms only for the electronics sector.

From the point of view of technology regimes, this finding seems to go against the intuition of the chemicals knowledge base as mote cumulative and closed to outsiders. On the basis of this intuition, one might expect that between firms diffusion is relatively slow for the chemicals sectors. A possible explanation for this paradox might be the relative homogeneity of the knowledge base between the three chemicals sub-sectors, and the heterogeneity of the knowledge bases between the electronics and telecommunications sectors. Indications for this difference between the two broadly aggregated sectors were already found in Table 5.2. Unfortunately, the number of firms and patents in the current sample is not sufficient to prepare a reliable breakdown of the data in Figure 5.5 for the sub-sectors.

Finally, we analyse the relationship between citations, distance and time together at the sector level. To do this, we start by dividing the potential citation pairs into four segments according to the two criteria within/between groups and zero/positive citations. We also calculate the distance between the two regions involved in the citation link. Then, for each of the four tesulting segments, we calculate the (unweighted) mean of the distance of all citation links in the segment. This will enable us to compare the average distance for positive citation links with that of zero citation links. In light of the theoretical discussion above, we would expect that the distance for the positive citation links would be lower than for the zero citation links. 

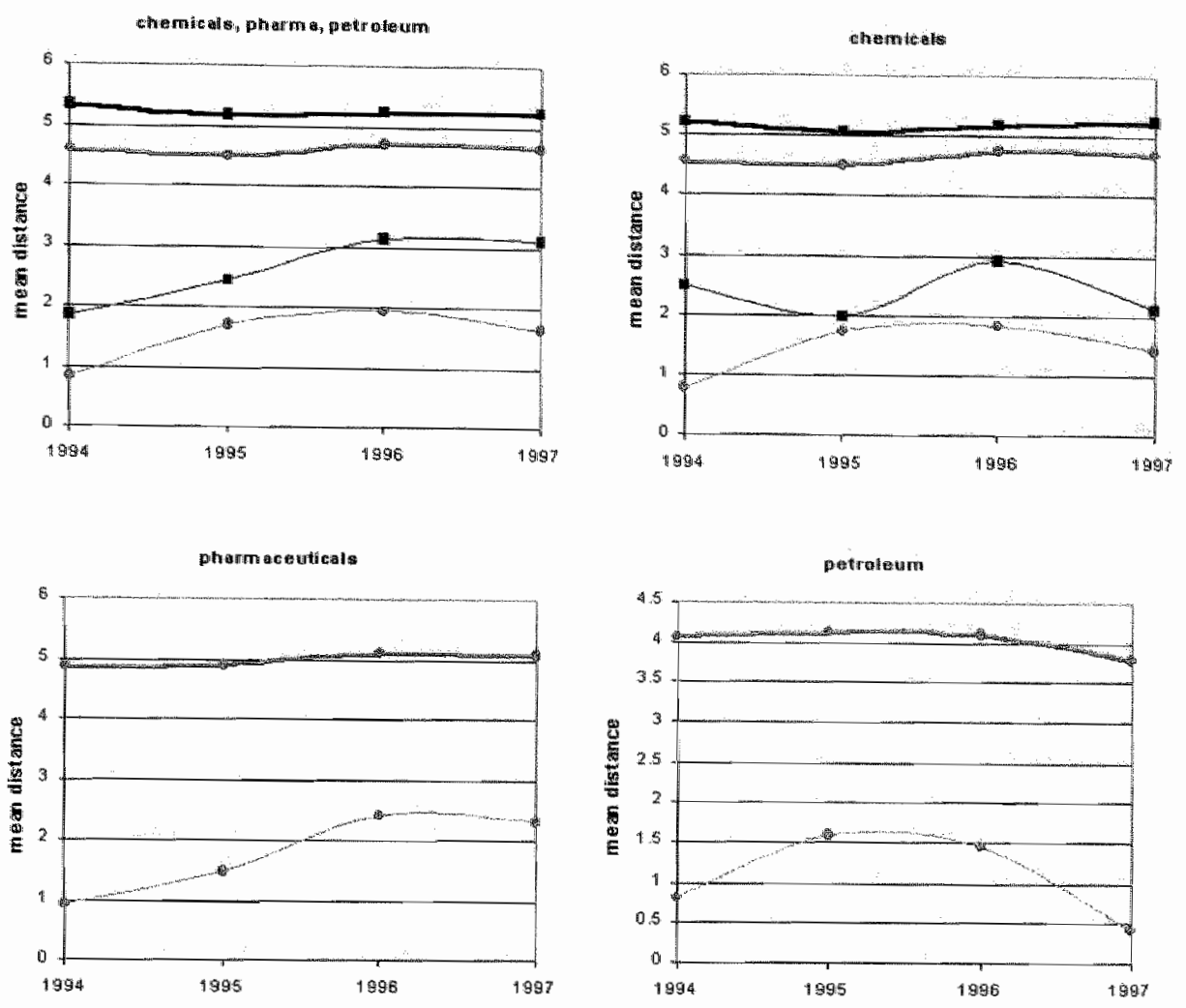

Figure 5.6. The impact of distance and time on patent citations, within and between MNEs, Chemicals.

In addition, we can look at the development of average distance over time within each segment of positive citations. Jaffe and Trajtenberg (1996) found that as time passes since the application of the cited patent, patent citations span a wider geographical distance. Such a phenomenon is broadly in accordance with the notion of knowledge diffusion as a spatial phenomenon (Hägerstrand 1967), implying that knowledge will first diffuse to spatial units close to where the knowledge originated, and subsequently diffuse to a larger spatial area. This theory nicely complements the vision of knowledge as a spatially sticky phenomenon that was discussed above. 
Figure 5.6 displays the results for the chemicals sectors. The thin lines indicate the development of average distance over time for the positive citations segments; the thick lines do the same for the zero citations segments. For pharnaceuticals and petroleum, the segment for positive citations between groups is too small to make the results reliable, so these lines are omitted from the graphs. The first result is that the lines for positive citations are well below the lines for zero citations for all cases. In other words, positive citation links show a lower mean distance between the two regions involved than zeto citation links. It must be noted, however, that the standard deviation of the distances within all segments is rather large. This suggests that a test for statistical significance of the difference between the mean distance of positive and zero citations would not reject the null hypothesis of equal means, although it is not quite clear which test could be used since the distributions of the distances involved appear to be non-normal (skewed to longer distances). On the other hand, the fact that for all four graphs we find the same result, namely that positive citations have lower mean distance than zero citations, is suggestive of a systematic tendency rather than a random phenomenon.

With regard to knowledge flows as a spatial process, the results are quite interesting. Statting with the graph for all three chemicals sectors together (upper left comer), we do observe an initially increasing mean distance. That this is not due to a change in the underlying distribution of patenting (as opposed to citation) activity over space is indicated by the fact that the lines for zero citations remain largely flat over time. Thus, we do find that, on average, knowledge diffuses first to nearby spatial units, and only later to regions further away. However, after two years, a peak is reached, and the graph levels off, and even starts to decline marginally. This general pattern is common between citations between groups and citations within groups, although the decline is more significant for the within groups citations.

The decline of average distance for the last year (1997) can be interpreted in terms of an assumed interaction between spatial distance and the specificity of knowledge for the regional innovation system. In such an interpretation, the flow of knowledge to spatial units further away (i.e., the increasing part of the curve) corresponds at the same time to a broadening of the field of application of the knowledge. This follows from an assumption that each tegional system has its own specific pattern of technological interests and applications. After the knowledge has become older and hence more obsolete, it looses its relevance to areas of application that are further away from the original field in which it was developed. This implies that the spatial reach of the knowledge flows declines, and hence that the curve would fall (or level off). Obviously, there is an element of speculation in this interpretation, but it would be possible to test this proposition in future research by looking at the technology classes in 
which citations occur, and by extending the time period of citations beyond the four years we have used here.

The observed general time pattern for the three chemicals sectors as a whole is, to the extent that data is available, more or less repented for the individual sectors. The one exception is the curve for between group citations in chemicals. This curve starts from a relatively high level of mean distance. However, it must be noted that this particular observation is based on only four citation links, and hence may be influenced by random factors. The eventual decline is strongest for within group citations in the petroleum sector. This sector also shows a rather early peak, i.e., at the year 1995. Finally, it is observed that all peaks of the observed curves for positive citations are well below the level indicated by the mean distance for zero citations.

Figure 5.7 displays the same indicators for the electronics sectors and motor vehicles. Computers has too little citations in either category to provide reliable results, for telecommunications it is only possible to calculate reliable results for within group citations. In the electronics graphs, the findings differ substantially between within group and between groups results. For the electronics sectors together as well as for the electronics sector in a nartow interpretation, between groups citations start off at a level that is above the mean distance for zero citations for that segment. The two curves then converge more or less to the mean distance of zero citations links. This result is obviously in contract to the expectations based on the theoretical discussion above. Closer inspection shows that this is largely driven by two firms: Nokia and Ericsson. Of the 187 between groups citations in electronics (narrowly defined), 87 have distance larger than 6 (which is about the mean distance of zero citations). Of these 87 cases, only 8 (or $9 \%$ ) do not involve either Nokia or Ericsson. Although these Scandinavian firms do a large part of their research abroad, their domestic patents citing other patents add long distance citations to the sample.

The other curves in the figure are consistent with the patterns already observed in the previous graphs. They show mean distances below the values for the corresponding category of zero citations, as well as the typical hillshaped pattern that was discussed above. Still, there are some differences between the various curves. In telecommunications and motor vehicles, the peak of the curves occur rather early, i.e., 1995, as opposed to 1996 or the other sectors.

Summarizing, we do find support for the hypothesis of spatial concentration of knowledge flows. In general, positive citation links between regions are characterised by lower mean distance between the citing and cited region than for potential citation links that do not lead to positive citations. This seems to be a tendency that is not specific to any of the sectors considered here, but instead occurs across the board of sectors and technologies. To the 


\section{CHAPTER V}
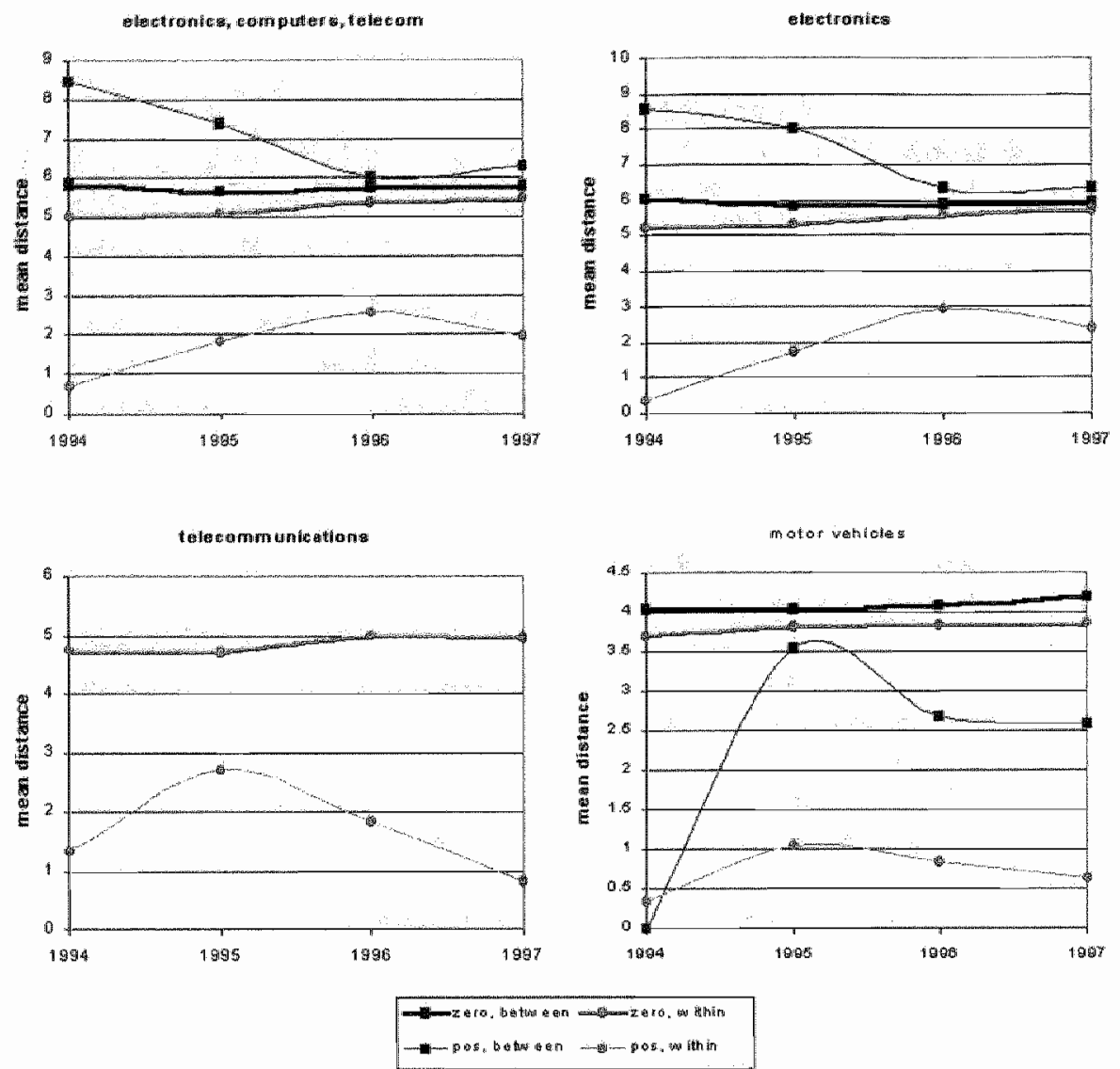

Figure 5.7. The impact of distance and the on patent citations, within and between MNEs, clectronics and motor vehicles.

extent that there are exceptions to this tendency (Nokia and Ericsson in electronics), firm specific factors seem to play a role in explaining these, rather than sector differences. We also find evidence for a spatial pattern of knowledge diffusion, i.e., at first citations occur at low distance, after which the spatial reach of the citation process increases. We do observe, however, a levelling off, or even reversal of this process. 


\subsection{Discussion and conclusions}

The aim of this chapter was to provide empirical evidence for the argument that even in an age of "globalisation", regional innovation systems matter. To this end, a database on patenting by 87 latge multinational enterprises (MNEs) from European regions was used. Several empirical findings stand out.

First, it was found that the degree to which the firms in the sample perform their R\&D in foreign countries varies. The data on this phenomenon are limited to European firms. Of these, the percentage of foreign patenting in total patenting varied between vittually zero and $100 \%$. The mean was around one fifth, which is comparable to previous findings in the literature (e.g., Le Bas and Sierra 2002).

Second, it was shown that a limited set of European regions attracts by far the largest part of (foreign) R\&D activity by the firms in our sample. Almost all these regions are located in the central part of Europe, more specifically in the United Kingdom, Ftance, Germany, Switzerland, the Netherlands and Belgium. Southern Europe attracts very little R\&D activity by the firms in our sample, and only some very limited number of Northern European regions rank high. We take this as evidence of the fact that regional technological capabilities (still) matter in the decision of our MNEs on where to locate (foreign) R\&D. We also observe differences in terms of where to locate between European, Japanese and US firms.

Third, we found evidence that the Blue Banana area (Hospers 2003) is still the most important growth area in Europe. We found only scant evidence for the Sunbelt area, and no support for the Yellow Banana area. Economic and institutional diversity is probably the most important explaining factor for the difference in growth rate between the Blue Banana area and the other European areas. The observed distribution of innovative activity across European regions is probably very stable over time, and is not very likely going to change in the near future.

Fourth, it was found that there is no clear linear or monotonic relationship between the size of a firm's R\&D activity and its tendency to spread over more locations. In fact, it was found that the firms with intermediate levels of activity are most likely to have a large spread over European regions.

Fifth, we tested whether or not technology flows as indicated by patent citations are localized in space. We analysed both flows between MNEs and flows within MNEs, i.e., between a firm's different regional locations. It was found that for all cases where enough data exists, knowledge flows within a MNEs have an important local component, i.e., that these flows are more intense between units of the firm that are nearby than between units of the firm that are further apart. Although we found differences in citation patterns 
between sectors, the spatial dimension of citations seemed to be largely invariant between sectors. The spatial concentration finding holds for most cases of knowledge flows between MNEs, although there are exceptions to the phenomenon in this case. Specifically, it was found that in electronics, the citation flows involving two large Scandinavian firms, i.e., Nokia and Ericsson can be chatacterized as "Iong distance". This shows that besides geographical factors, knowledge flows are also characterized by a number of other, often firm-specific factors.

Finally, we used the citation data to test the hypothesis that knowledge first diffuses to nearby locations, and subsequently reaches a larger spatial realm. We found evidence supporting this hypothesis, again for citations within MNEs and between MNEs, although the case was stronger for citations within MNEs. Moreover, we found that during the early stages of the knowledge diffusion process, the spatial reach of knowledge increases, but after a while (usually two years), the reach declines again. 'This was attributed to the regional specificity of knowledge, although more empirical work needs to be done to substantiate this argument further.

Overall, the results support the conclusion that regional innovation systems in Europe still matter, at least as far as large MNEs are concerned. This is a conclusion that both has important policy implications, and has implications for further research in the field. With regard to policy, one might expect that European regional cohesion be at stake, especially because of the localised nature of knowledge flows. This means that there might be selfreinforcing tendencies for rapid growth based on the application of new knowledge. However, further research is necessary to see what role is played in this by smaller firms than the ones in our sample, and whether or not the localised nature of knowledge flows is also relevant for knowledge flows related more to production than the flows that we analysed. 


\section{CHAPTER 6 \\ Appraisal}

\subsection{Introduction}

In this chapter we will summarise the main points of the thesis. We will seek to answer our central research question and try to reflect on the resuits of our analyses. We will elaborate on the strengths and weaknesses of the thesis and provide suggestions for further research.

The purpose of this thesis was to increase our current understanding of the knowledge flows that occur between multinational companies. In order to do this we empirically tested several hypotheses. We first investigated the factors that influence the knowledge flows between multinational companies. We tried to identify the particular factors and their impact on the knowledge flows. Next we analysed the influence of strategic technology alliances on knowledge flows between multinational companies, and on organisational learning in general. Finally we studied how spatially bounded knowledge flows are, and how they spread over distance and over time. The answers to the different specific research questions will enable us to answer the central research question as postulated in the first chapter.

In the remainder of this chapter we will summarise the empirical results from our research and reflect on their major conclusions. We will also present an overall answer to the central research question of this thesis (section 6.2). We will summarise without giving elaborate references to underlying theories, since this is already done in the individual chapters themselves. We will also elabotate on the strengths and weaknesses of this thesis (section 6.3), and finally provide suggestions for further research (section 6.4).

\subsection{Overall summary and conclusions}

For the empirical part of this thesis we made use of an extensive database that was constructed especially for the purpose of this thesis (see also chapter 2). For the construction of this database we made use of several existing databases of which the European Patent Office database, the Dun \& Bradstreet linkages database and the MERIT-Cooperative Agreements and Technology Indicators database are the most noteworthy. Because of the size and the 
specificity of the database we were now able to test relationships that were not testable before. We have detailed information on 171 multinational firms working in the medium- or high-tech sectors of the economy. Especially the combination of the different databases has proven to contribute considerably to our research.

\subsubsection{Factors influencing knowledge flows}

We started by investigating the factors that influence the knowledge flows between multinational companies. Based on the resource-based view of the firm (Penrose 1959; Wernerfelt 1984) and the dynamic capabilities view of the firm (Teece, Pisano et al. 1997) we know that resources and especially the renewing of firm resources are very important for company success. Renewing of firm capabilities is particularly important in modern day economic environment with its constantly changing competitive edges. For this renewing of firm resources organisational learning is a related and very important concept. Firms can only keep their resources dynamic when they are able to learn from other companies, or when they have enough resources to conduct research themselves. Since organisational learning turns out to be of such vital importance for the renewing of the dynamic capabilities of the firm and consequently for the firm's economic performance, it would be interesting to shed light on how this mechanism of learning works by investigating how knowledge flows outside firm boundaries. In chapter 3 we therefore researched which factors influence the knowledge flows between multinational companies, and in what way. We followed the reasoning of Jaffe and Trajtenberg (1998) in that patents are pieces of knowledge, and patent citations are a proxy for a knowledge flow.

For knowledge to flow between companies two factors are very important. First firms must be willing to use the new knowledge and if necessary adapt it to their internal needs (Trott, Cordey-Hayes et al. 1995). Second even if firms are willing to incorporate outside knowledge they must also be able to absorb the new knowledge (Cohen and Levinthal 1990). Put differently they must be able to understand the new knowledge. This then supposes a certain relatedness between the new knowledge and the knowledge that already resides within the firm. Firms can only learn from new knowledge if they are able to relate it to already existing knowledge within the firm. This absorptive capacity of the firm is therefore considered to be the first important factor in firm learning. We specified absorptive capacity as the total amount of knowledge available in the firm, measured as the total pool of patents of the firm, the firm specific knowledge base. We therefore hypothesised that a larger 
firm specific knowledge base will increase the knowledge flows to and from a company.

The total collection of patents of a firm can be considered a static measure of absorptive capacity; we should however also incorporate dynamic factors influencing the absorptive capacity. One of the most important dynamic factors influencing the specific knowledge base of a firm is the R\&D spending of the firm. Besides using $R \& D$ for direct innovations firms also use it to develop their capabilities further and to assimilate and exploit external knowledge (Cohen and Levinthal 1989). So not only is investing in R\&D a means of internal leaming it also helps in learning from the environment. "These firms will thus be better at incorpotating knowledge flows from the environment. On the other hand can we expect these firms to be more interesting for other firms as a supplier of external knowledge. We would thus expect more knowledge flows to and from a company with increasing R\&D spending.

As R\&D spending can be considered a means of internal learning, firms can also learn directly from the environment. Nowadays external learning is quite often done via a strategic alliance. More and more, alliances are used to learn from the allying partners (Hagedoorn and Schakenraad 1990; Hagedoom $1993 \mathrm{~b}$ ), and firms especially establish alliances for the exchange of knowledge. The advantage of learning via a strategic alliance is the speed at which it can be accomplished compared to intemal developments via R\&D. We thus expected more knowledge flows to a company that is involved in an alliance, but we also expected more knowledge flowing from the company to the allying partner(s).

From our statistical analysis we found strong support for a positive influence of the three factors we investigated on the knowwledge flows to and from the company. This thus means that a larger specific knowledge base of a company will generate more knowledge flows to and from the company. Also firms that invest more in $R \& D$ will experience more knowledge flows to and from the company. And last, companies with more alliances will have more knowledge flows in and out of the company. We also found that the larget the firm the more on average they will cite other firms. However based on our results we were unable to make any comments on the influence of firm size on the knowledge flowing from the firm to other firms.

Overall we concluded that knowledge flows, which ate very important for company performance, are positively influenced by the three factors we researched, namely absorptive capacity, $R \& D$ intensity and number of alliances. Since knowledge flows are so important for organisational learning and thus for overall company success it is very important to understand the factors influencing these knowledge flows. For firms working in high-or medium-tech sectors it is thus very important to work on their absorptive capacity via investments in $\mathrm{R} \& \mathrm{D}$ and through the forming of alliances. 


\subsubsection{The influence of strategic technology alliances}

Next we tried to deepen our understanding of the influence of strategic technology alliances on the knowledge flows to and from the company. We again build on the resource-based view of the firm and on the dynamic capabilities view, but we extended our theoretical basis with the knowledgebased view of the firm (Grant and Baden-Fuller 1995; Foss 1996). This last theory provides a new explanation for the observed trend towards collaborative agreements between firms. Firms have been shown to collaborate, among other things, to be able to get access to the knowledge of other firms (Hagedoorn 1993b). Given the increasing importance of external knowledge appropriation by means of strategic alliances, it is of major importance to understand the nature of strategic technology alliances and to take a closer look at the impact of firm collaborations on organisational leaning.

We first looked at the shape of the relationship of learning between firms within strategic alliances. Again we turned to the concept of absorptive capacity (Cohen and Levinthal 1990). If firms want to absorb the knowledge of their allying partner, we can expect that for learning to take place, there has to be at least some relatedness between the two firms' general knowledge bases. The general knowledge base of a firm we defined as the total amount of patents of the firm plus the patents cited in these patents. The absorptive capacity of a firm we defined as the overlap in the general knowledge bases of the two allying firms. We then expected that a very small overlap in knowledge bases would have a negative influence on the learning in the alliance. Firms need to have at least a minimal absorptive capacity in order to be able to learn from their allying partner. If the overlap increases we expected also the learning to increase, but not indefinitely. There will probably be an optimal point of overlap that will facilitate the learning best. If the overlap would be too large we would expect that the firms will not be able to learn much from their partner, because they posses the knowledge alteady themselves. We therefore hypothesised an inverted U-shaped relationship between the overlap in general knowledge bases of the firms and the learning taking place in the alliance.

Besides using alliances to learn from the allying partners, alliances are also established for many other purposes (Hagedoorn 1993b). In complementary allinces (Teece 1986) the aim is not to learn from the partner, but on the contrary they are established so that every partner can specialise in his own field of expertise; the firms complement each other. If we look back at the discussion we had before about the general knowledge base overlap, then we would expect this overlap in a complementary alliance to decrease or at least not increase. Firms specialise in that case in different fields of knowledge and are not trying to learn from each other's technical knowledge. We therefore expected that we would observe a significantly greater increase in general 
knowledge base overlap, and thus more learning, between firms allying in a learning alliance as opposed to a complementary alliance, or firms not working together in an alliance.

A further point of interest is the difference in learning between strong and weak tie alliances. Weak ties are considered to be more important for the diffusion of unrelated knowledge, and strong ties for the diffusion of related knowledge. In weak tie relationships firms are able to learn from dissimilar knowledge bases whereas in the case of strong ties they are able to deepen their existing knowledge base. Firms would be able to learn more in a weak tie relationship, since the knowledge base of the partner firm is more dissimilar, but the lack of "social capital" or trust might inhibit learning. Opportunism can be expected to be larger in weak tie relationships as opposed to strong tie relationships and this would prevent learning from taking place. In strong ties opportunistic behaviour affects reputation more as opposed to a weak tie situation. So while firms could potentially learn more in a weak tie situation, the fear for opportunistic behaviour might prevent learning from taking place: Consequently we expected more learning to take place in a strong tie relationship as opposed to a weak tie relationship.

From our statistical analysis we found corroborating evidence for an inverted U-shaped relationship between the overlap in knowledge base between the allying partners before the alliance and the learning taking place in the alliance. This means that there is an optimal overlap at which the learning is maximal. Too little or too much overlap might inhibit the knowledge from flowing. It also turned out that firms working together in a learning alliance showed more knowledge flows amongst each other, compared to firms not working together in a learning alliance, or not working together in an alliance. Furthetmore we found, contrary to what we expected, that weak ties turned out to be more important for learning in strategic alliances than strong ties. Complementarity between firms thus seems to outweigh trust in strategic alliances. The firms in our dataset might also be suffering from "overembeddedness". Overall, we concluded that strategic alliances ate an important vehicle for knowledge acquisition, but that the selection of the allying partner turns out to be of critical importance for effecive knowledge exchange. For the partner selection process especially the knowledge base overlap between the partners turns out to be very important, along with the strength of the tie between the firms.

\subsubsection{The spatial dimension of knowledge flows}

Further we investigated the spatial pattern of patenting by the world's largest multinational enterprises (MNEs). In the business literature on 
locational behaviour of R\&D activities by MNEs there is no single unique point of view on this subject. Onlly recently has business literature come to the conclusion that "globalisation" is an important phenomenon effecting R\&D activities by MNEs (e.g. Dunning and Narula 1995; Patel and Vega 1999). A theoretical explanation for this trend is two-fold. First, firms might use their home-base developed technologies and use them in a foreign location to be able to adapt their products and technologies to the local market, called assetexploiting behaviour (Dunning and Narula 1995). Second, firms might try to tap into local knowledge bases in order to expand their existing technological capabilities, this is called asset-seeking behaviour (Dunning and Narula 1995). Firms than use the local knowledge base to develop new capabilities, complementary to their already existing capabilities. Only this second explanation is linked to spatial concentration of $R \& D$ activities, the first one is not. If firms were able to develop all knowledge in their home base there would be no need for foreign R\&D activities, but apparendly geography matters. For this to be true we thus had to pre-assume that knowledge bases differ between regions, and that it is not easy to tap into these knowledge bases from a distance. It would therefore be interesting to investigate the concentration of patenting activitics over different regions in order to be able to make a statement about the spread of R\&D activities by MNEs.

Continuing this line of argument, the choice for a particular region in terms of foreign $R \& D$ location depends on two factors: the regions local knowledge base, and the extent to which the firm is able to tap into this knowledge base (Morgan 2004). R\&D activities might be localised for two reasons. First, there is the argument of agglomeration economies where certain resources, like skilled labour, are only available in (a) certain region(s). The second reason for the spatial nature of knowledge is related to the tacit nature of knowledge itself, which necessitates nearness. Depending on whether assetexploiting or asset-seeking behaviour is more important, innovative activities might be spread more evenly over regions, or be more concentrated in a limited number of regions. We thus wanted to investigate which of the two prevails for the firms in our dataset.

The overall picture that emerges from our research is that most firms have substantial foreign patenting activities, but these are concentrated in a limited number of regions, both from a European spatial perspective, and from the point of view of the number of locations per firm. There are however also some firms, which patent from a large number of regions and source a large shate of their total patents from foreign countries. We additionally compared our results with other researches on the spread of innovative activity of MNEs across Europe. We found that our results are inline with these other researches (e.g. Cantwell and Iammarino 2001; Hospers 2003). Especially the European area termed the "Blue Banana" is also found in out results to be the most 
important area for innovative activity in Europe. This division across regions is moreover expected to be rather stable over time.

We further wanted to investigate the mechanism that may lead to this concentration. To do this we first operationalised two additional dimensions: distance and knowledge flow. Distance is defined based on the NUTS classification, where we measured distance by counting the minimal number of borders that have to be crossed to go from one region to the other. Knowledge flows are again measured as a patent citation (Jaffe, Trajtenberg et al. 1993; Jaffe, Fogarty et al. 1998).

The first noteworthy finding is that knowledge flows between the MNEs in our sample as indicated by patent citations are a relatively small phenomenon. We also found important differences between sectors.

Secondly, we seem to have evidence that knowledge diffuses more tapidly within firms than between firms only for the electronics sectors. A possible explanation for this finding might be the relative homogeneity of the knowledge bases between the three chemical sectors and the relative heterogeneity of the knowledge bases in the electronics sectors.

Next we analysed the relationship between citations, distance and time at the sectoral level. We wanted to investigate the spatial concentration of knowledge further. We also expected that as time passed, patent citations would span a wider geographical distance (Jaffe and Trajtenberg 1996). For the chemicals sectors we indeed found that knowledge flows are spatially concentrated. For these sectors we also found, on average, that knowledge diffuses first to nearby spatial units, and only later on to regions further away. For the electronics sectors the results are consistent with the pattern observed in the chemicals sectors. Only here the results of the between group citations differ substantially, but this is caused by two firms with many long distance citations. For the telecommunications and motor vehicles sector we saw that the curves peaked one year earlier as compared to the other sectors. For the pharmaceutical, the petroleum and the telecommunications sectors we did not have enough information to draw the between patent citation links.

On the whole we come to the following conclusions. First, the degree to which firms perform $\mathrm{R} \& \mathrm{D}$ in foreign countries varies considerably for the firms in our sample, but on average they petform a considerable amount of R\&D abroad. Second it is shown that for the firms in our dataset only a few European regions attract the largest part of (foreign) R\&D activity. Most of these European regions are in the central part of Europe, in an atea also called the Blue Banana. Third, we did not find a clear linear or monotonic relationship between the size of a firm's R\&D activity and the tendency to spread the R\&D over more locations. We actually found that firms with an intermediate level of R\&D activity have a higher chance of having a larger spread over the European regions. Fourth, we investigated if knowledge flows are localised in space, we 
tested this both between en within MNEs. What we found was that proximity does matter for knowledge flows, again both between and within MNEs, although there are also firm-specific factors that seem to play a role here. Lastly we tested if knowledge first diffuses to nearby locations before reaching a larger spatial area. We did indeed find support for this hypothesis although stronger support for within knowledge flows than for between knowledge flows. The spatial reach turned out to expand first and to contract later on, uscially after two years. Probably this is due to the regional specificity of the knowledge, although more research is needed here. We thus found evidence for the hypothesis of spatial concentration of knowledge flows, and we also found evidence for a spatial pattern of knowledge diffusion. On the whole, our results show that regional innovation systems in Europe still matter, which could have serious policy implications; the European regional cohesion might be at stake. However, also here further research is needed.

\subsubsection{Overall conclusions}

When looking back at our central research question in chapter 1: What is the nature of knowledge flaws berween whitinational companies? We can conclude that knowledge flows to and from multinational companies are positively influenced by the level of absorptive capacity of the firm, the R\&D intensity and the number of alliances the company has had. The notion of the importance of absorptive capacity and R\&D intensity on organisational learning is confirmed in many studies, some of the most notable are Cohen and Levinthal (1990), and Lane and Lubatkin (1998). Strategic technology alliances, despite their high failure rate (Duysters, Kok et al. 1999), in general turn out to be an effective wehicle for organisational learning and knowledge transfer (e.g. Hagedoorn and Schakenraad 1990; Hagedoorn 1993b; Mody 1993; Khanna 1998; Lane and Lubatkin 1998). Especially important in this respect turned out to be the knowledge base overlap between the allying firms. Dyer and Singh come to the same conclusion (Dyer and Singh 1998). Learning in strategic technology alliances furthermore follows an inverted U-shaped pattern, depending on the knowledge base overlap between the allying partners before the alliance. This means that the learning will first increase with increasing knowledge base overlap, reach a maximum and then decrease again. Some recent studies suppose a linear relationship between knowledge base overlap and learning taking place (e.g. Mowery, Oxley et al. 2002). Our results are however inline with earlier research on the relationship between the relatedness of acquired and acquiring knowledge bases and the innovative performance of companies in case of an acquisition by Ahuja and Katila (2001). Alliances established especially for the purpose of knowledge transfer also showed a larger increase 
in knowledge base overlap than do firms in a non-learning alliance, or firms not involved in an alliance. Similar tesults were found by for instance Nakamura, Shaver et al. (1996) and by Mowery, Oxley et al. (1996 and 2002). Furthermore weak tie relationships in alliances turned out to be better suited for knowledge transfer than strong tie relationships. This result is in contrast with our expectations but could indicate that for alliances in this environment complementarity between. firms seems to outweigh trust. It might also indicate that the firms in our dataset are suffering from "over-embeddedness".

Knowledge flows also turned out to be spatially concentrated, both within and between MNEs. Earlier research by Cantwell and Iammarino (2001) showed more or less similat results, although our results are more specific. If we compare our results to the "Blue Banana" concept (Hospers 2003) we find that this European area is also according to our results the most important area for innovative performance of MNEs in Europe. When knowledge flows between regions, it will follow a spatial diffusion pattern, flowing first to nearby regions and later on to regions further away. This is inline with research of Hägerstrand (1967), and Jaffe and Trajtenberg (1996). We do however experience that the spatial reach will increase for about two years, after which a maximum is reached and the spatial reach will decrease, possibly brought about by the regional specificity of knowledge and by the fact that knowledge becomes obsolete over time.

When answering our central research question we can thus conclude that knowledge flows turn out to be concentrated in certain geographical areas, and follow a spatial diffusion pattern (at least for Europe). Increasing the absorptive capacity of the firm, brought about by relatively high $R \& D$ investments and alliance formation, enhances knowledge flows. Strategic technology alliances in this respect turn out to be an excellent vehicle for organisational learning since they facilitate knowledge flows between companies.

For firms that want to be on the cutting edge of new technological developments it is thus very important to be in, or as close as possible to, the area(s) where the necessary knowledge is in order to be able to tap into this knowledge pool in time. Especially helpful in gaining new knowledge are strategic technology alliances and own R\&D developments, both facilitate the absorption of new knowledge. Since knowledge flows turn out to be so wery important for our economy, and for individual company success, it is therefore very important to understand the nature of such flows. Hopefully this thesis car be of help in enlightening the nature of knowledge flows in our economy. 


\subsection{Strengths and limitations, and suggestions for future research}

Of course this study, like any other, has its strengths and limitations. We will touch on the most important here. In this section we also want to offer suggestions for further research. Part of these suggestions were already done in earlier chapters, but are summarised here. Some suggestions are more general in nature and were not presented earlier.

One of the most obvious strengths of this thesis is the extensive database that is constructed and used to test the hypotheses. Especially the combination of two vast datasets one on patents, the EPO database, and the other on strategic alliances, the MERIT- CATI database is important in this respect. The database contains detailed information on 171 multinational firms covering the medium- and high-tech sectors of the 500 largest companies in the world. Because of the size of the database, the conclusions ate applicable to a large part of the economy. We are not restricted to just one or a few sectors. Although we researched many firms in many sectors, we restricted the research to only multinational enterprises. This means that our conclusions are restricted to only MNEs; we can say next to nothing about the knowledge flows between smaller firms although they might be equally important. For future research it would thus be interesting to extend our database to smaller firms and to replicate our research. This could be a valuable complement to our research.

We also constructed our main database only for 1998; for future research it would be very interesting to construct the same database for more years. Although this is very time consuming, it could strengthen and help fine-tune our results considerably. As discussed before in chapter 2 this database construction is however still under construction.

Patent data further has its flaws and drawbacks as discussed in chapter 2. The most noticeable difficulties with patent data are that some inventions are never patented; that there are considerable differences in patenting behaviour between sectors; that all patents, disregarding their importance, receive the same weight; and that patents only cover codified knowledge. Most of these weaknesses were however accounted for one way or the other (see chapter 2 for the accountability).

Another important strength of the thesis lies in the high degtee of specificity of the database concerning the patenting data. We are able to exclude self-citations from the other patent citations, and are therefore able to distinguish between within and between MNEs knowledge flows with a very small error level. Although we are able to distinguish between inter- and intrafirm knowledge flows, in our research we mostly looked at inter-firm knowledge flows. Based on our dataset it would also be interesting to look at 
the intra-firm knowledge flows and to see how knowledge enters the MNE, and at what places, and how it seeps further through the MNE (if it seeps through anyhow).

We further distinguished the most important factors influencing the knowledge flows to and from multinational enterprises, and researched the direction and magnitude of their influence. Concerning these factors we have two suggestions for future research. The first suggestion is that more research is needed into the direct influence of the observed factors. The three factors, absorptive capacity, $R \& D$ intensity and number of former alliances, ate now each treated as one homogeneous factor. We might be able to clarify their influence even better by looking in more detail at these factors (we did this only for the factor "number of alliances"). Secondly we would like to suggest to direct research on the discovering of other factors. We very probably have found the most important factors, but might have overlooked smaller, but still important other factors.

We have also proven that the relationship of the learning taking place in a learning alliance follows an inverted U-shaped relationship, based on the prealliance knowledge base overlap. We also proved that strategic technology alliances are an important vehicle for knowledge transfer and organisational learning. We have three suggestions for further research. The first suggestion would be to replicate the research using alliance data for other years than 1993 , to be able to control for yearly fluctuations. Furthermore we could enlarge the research by including so called complementary alliances, to better test the difference in learning between the different alliance types. Another measure for the strength of the tie in the alliance relationship could be another improvement to the current research.

Further, in this thesis we were able to test the theoretical deducted assumption of spatial concentration and spatial diffusion of knowledge flows for the whole of Europe, for multinational enterprises. To our knowledge this was the first time that this relationship has been empirically tested, based on the patent activity of MNEs. Concerning this relationship we would suggest to investigate the causal structure between concentration of innovative activities and the existence of localised knowledge flows. Econometric methods might be able to shed light on this matter. Concerning the knowledge diffusion process, more research is needed into the declining of the reach after an initial period of increase and the link with regional specificity of knowledge. Possible suggestions could be to include the technological classes in which the citations occur, and by extending the time period over which the patent citations are included beyond the four years we are using.

An additional important point is the use of European patent data in this thesis, which makes our study an important complement to existing research, 
based on US patent data. The European patent data might also be more relevant for research in a European setting.

A further suggestion for future research is the use of performance measures for the firms we are researching. It would be interesting to combine the results of our study with company performance measures to see if firms that have more knowledge flows actually also perform better. It would also be very interesting to use qualitative research methods in order to test some of our hypotheses by for instance interviewing the appropriate people in MNEs. Both could be important complements to our current tesearch. 


\section{Appendices}

Appendix 1: Company names for the companies in our dataset.

\begin{tabular}{|l|}
\hline Company name \\
\hline AB Volvo \\
\hline ABB Asea Brown Boveri Ltd \\
\hline Abbott Laboratories \\
\hline Aerospatiale \\
\hline Akzo Nobel NV \\
\hline Alcatel \\
\hline Allied Signal Inc. \\
\hline Aluminum Company of America \\
\hline American Home Products Corporation. \\
\hline Ameritech Corporation \\
\hline Amoco \\
\hline Asahi Chemical Industry Co. Ltd. \\
\hline Ashland Inc. \\
\hline AT\&T Corporation \\
\hline Atlantic Rich field Company \\
\hline BASF AG \\
\hline Bayer AG \\
\hline Bayerische Motoren Werke AG \\
\hline BCE Inc. \\
\hline Bell Atlantic Corpotation \\
\hline Bellsouth Corporation \\
\hline Bristol-Myers Squibb Company \\
\hline British Aerospace plc \\
\hline British Steel plc \\
\hline British Telecom plc \\
\hline BTR plc \\
\hline Cable \& Wireless plc \\
\hline Canon Inc. \\
\hline Caterpillat Inc. \\
\hline Cea-Industrie \\
\hline Chevton Corporation \\
\hline Chrysler Corporation \\
\hline Chinese Petroleum Corpotation \\
\hline Compag Computer Corporation \\
\hline Cosmo Oil Co. Ltd. \\
\hline Daewoo Corporation \\
\hline
\end{tabular}




\begin{tabular}{|l|}
\hline Daimler-Benz AG \\
\hline DDI Corporation \\
\hline Deere \&CCompany \\
\hline Degussa AG \\
\hline Dell Computer Corporation \\
\hline Den Norske Stats Oljeselskap A.S. \\
\hline Denso Corporation \\
\hline Deutsche Telekom AG \\
\hline Du Pont de Nemours and Company Inc. \\
\hline Eastman Kodak Company \\
\hline Electrolux AB \\
\hline Elf Aquitaine \\
\hline Emerson Electric Co. \\
\hline ENI S.p.A. \\
\hline Exxon Corporation \\
\hline Fiat \\
\hline Ford Motor Company \\
\hline France Telecom S.A. \\
\hline Fried. Krupp AG Hoesch-Krupp \\
\hline Fuji Heavy Industries Ltd. \\
\hline Fuji Photo Film Co. Ltd. \\
\hline Fujitsu Limited \\
\hline GEC General Electric Co.plc \\
\hline General Electric Company \\
\hline General Motors Corporation \\
\hline Glaxo Wellcome plc \\
\hline GTE Corporation \\
\hline Henkel KgaA \\
\hline Hewwlett-Packard Company \\
\hline Hitachi Ltd. \\
\hline Hoechst AG \\
\hline Honda Motor Co. Ltd. \\
\hline Hyundai Motor \\
\hline Idemitsu Kosan Co. Ltd. \\
\hline Imperial Chemical Industries plc \\
\hline Indian Oil Corporation Limited \\
\hline Intel Corporation \\
\hline IRI \\
\hline Suzu Motors Ltd. \\
\hline
\end{tabular}




\section{APPENDICES}

\begin{tabular}{|c|}
\hline Japan Energy Corporation \\
\hline Johnson \& Johnson \\
\hline Johnson Controls Inc. \\
\hline Kawasaki Steel Corporation \\
\hline Kobe Steel Ltd. \\
\hline Komatsu Ltd. \\
\hline Koninklijke KPN NV \\
\hline LG Electronics \\
\hline Lockheed Martin Corporation \\
\hline Lucent Technologies \\
\hline$M A N A G$ \\
\hline Mannesmann $A G$ \\
\hline Matsushita Electric Industrial Co. Ltd. \\
\hline Matsushita Electric Works Ltd. \\
\hline Mazda Motor Corporation \\
\hline MCT Worldcom Corporation \\
\hline Merck \& Co Inc. \\
\hline Metallgesellschaft $A G$ \\
\hline Minnesota Mining and Manufacturing Company \\
\hline Mitsubishi Chemical Corporation \\
\hline Mitsubishi Electric Corporation \\
\hline Mitsubishi Heavy Industuies Ltd. \\
\hline Mitsubishi Materials Corporation \\
\hline Mitsubishi Motors Corporation \\
\hline Mobil Corporation \\
\hline Monsanto Company \\
\hline Montedison S.p.A. \\
\hline Motorola Inc. \\
\hline NEC Corporation \\
\hline Nippon Oil Co. L.td. \\
\hline Nippon Steel Corporation \\
\hline Nippon Telegraph \& Telephone Corporation \\
\hline Nissan Motor Co. Led. \\
\hline NKK Corporation \\
\hline Nokia Corporation \\
\hline Norsk Hydro ASA \\
\hline Northrop Grumman Corporation. \\
\hline Novarris group \\
\hline Occidental Petroleum Corporation \\
\hline PetroFina S.A. \\
\hline
\end{tabular}




\section{APPENDICES}

\begin{tabular}{|l|}
\hline Petröleo Brasilieto S.A. \\
\hline Petroleos de Venezuela S.A. \\
\hline Petroliam Nasional Berhad \\
\hline Peugeot S.A. \\
\hline Pfizer Inc. \\
\hline Phillips Petroleum Company \\
\hline Pohang Iron \&Steel Co. Ltd. \\
\hline Raytheon Company \\
\hline Renault \\
\hline Repsol S.A. \\
\hline Rhone-Poujenc \\
\hline Ricoh Company Ltd. \\
\hline Robert Bosch GmbH \\
\hline Roche Holding Ltd. \\
\hline Rockwell International \\
\hline Royal Dutch/ Shell Group \\
\hline Royal Philips Electronics \\
\hline Samsung Corporation \\
\hline Sanyo Electric Co. Ltd. \\
\hline SBC Communications Inc. \\
\hline Sharp Corporation \\
\hline Showa Shell Sekiyu K.K. \\
\hline Siemens AG \\
\hline SK \\
\hline Smithkline Becham plc \\
\hline Sony Corporation \\
\hline Sprint Corporation \\
\hline Sumitomo Metal Industries Ltd. \\
\hline Sunoco Inc. \\
\hline Surzuki Motor Corporation \\
\hline Swiss post \\
\hline Telecom Italia S.p.A. \\
\hline Telecomunicacoes Brasileiras S.A. \\
\hline Telefonakriebolaget Lm Ericsson \\
\hline Telefonica S.A. \\
\hline Telstra Co.Ltd. \\
\hline Texaco Inc. \\
\hline Texas Instruments Incorporated \\
\hline Textron Inc. \\
\hline The Boeing Company \\
\hline
\end{tabular}


APPENDICES

\begin{tabular}{|l|}
\hline The British Petroleum plc \\
\hline The Coastal Corporation \\
\hline The Dow Chemicals Company \\
\hline Thyssen AG \\
\hline Tosco Corporation \\
\hline Toshiba Corpotation \\
\hline Total S.A. \\
\hline Toyota Motor Corporation \\
\hline TRW Inc. \\
\hline United Technologies Corporation \\
\hline US West Inc. \\
\hline Usinor \\
\hline USX Corporation \\
\hline Volkswagen AG \\
\hline Xerox Corporation \\
\hline
\end{tabular}




\section{APPENDICES}

Appendix 2: Descriptive statistics for the firms in the dataset.

\begin{tabular}{|l|c|c|c|c|}
\hline Variable & Mean & $\begin{array}{c}\text { Standard } \\
\text { Deviation }\end{array}$ & Minimum & Maximum \\
\hline $\begin{array}{l}\text { Number of } \\
\text { patents }\end{array}$ & $1,838.05$ & $2,721.88$ & 0 & 18,438 \\
\hline $\begin{array}{l}\text { R\&D } \\
\text { expenditures } \\
\text { (\$millions) }\end{array}$ & 728.59 & 964.77 & 2 & 6827 \\
\hline $\begin{array}{l}\text { Revenues } \\
\text { (\$millions) }\end{array}$ & $27,301.35$ & $25,645.35$ & 8,968 & 178,174 \\
\hline $\begin{array}{l}\text { Number of } \\
\text { employees }\end{array}$ & $89,273.94$ & $83,828.97$ & 2,803 & 608,000 \\
\hline $\begin{array}{l}\text { Number of } \\
\text { subsidiaries }\end{array}$ & 163.16 & 141.50 & 2 & 822 \\
\hline
\end{tabular}




\section{References}

Accenture (1999). "Dispelling the myths of alliances." Ontlook Special Edition.

Acs, Z. J. and D. B. Audretsch (1988). "Innovation in large and small firms: An empirical analysis." The American Economic Review, 78(4): 678-690.

Acs, Z. J., L. Anselin, et all. (2002). "Patents and innovation counts as measure of regional production of new knowledge." Researreb Polig, 31: 1069-1085.

Ahuja, G. and R. Katila (2001). "Technological acquisitions and the innovation performance of acquiring firms: A longitudinal study." Strategic Management Journal, 22: 197-220.

Albert, M. B., D. Avery, et al. (1991). "Direct validation of citation counts as indicators of industrially important patents." Researhs Poligy, 20: 251-259.

Almeida, P. and B. Kogut (1997). "The exploration of technological diversity and the geographic localization of innovation." Small Business Economics 9: 21-31.

Anand, B. N. and T. Khanna (2000). "Do firms learn to create value? The case of alliances." Strategic Management Journal, 21: 295-315.

Anderson, H. C. (1848). Det er ganske wist. H.C. Andersens Eventyr. P. Hoybe. Copenhagen, Forlaget Notabene: $72-75$.

Appleyard, M. M. (1996). "How does knowledge flow? Interfirm patterns in the semiconductor industry." Stratagic Management Jotrmal, 17(Winter special issue): $137-154$.

Archibugi, D. (1992). "Patenting as an indicator of technological innovation: a review." Science and Public Policy, 19(6): 357-368.

Archibugi, D. and M. Pianta (1996). "Measuring technological change through patents and innovation surveys." Technovation, 16(9): 451-468.

Arora, A. (1995). "Licensing tacit knowledge: Intellectual property rights and the market for know-how." Economics of Innovation and New Tectsnology, 4: 41-59.

Arundel, A. and I. Kabla (1998). "What percentage of innovations are patented? empirical estimates for European firms." Research Poligy, 27: 127-141. 


\section{REYERENCES}

Arundel, A. (2001). "The telative effectiveness of patents and secrecy for appropration." Researob Polig, 30:611-624.

Badaracco, J. L. (1991). The knowledge link. How firms compete throwgh strategh allances. Boston MA. Harvard Business School Press.

Barkema, H.G., O. Shenkar, et at (1997). "Working abroad, working with others: how furms leatn to operate international joint ventures." Academy of Managentent Joman, $40(2): 426-442$.

Bamey, J. (1991). "Firm resounces and sustained competitive advantage." Jownal of Managenent $17(1): 99-120$.

Basberg, B. I. (1987). "Patents and the measurement of technological change: A survey of the literature." Researd Poliog, 16:131-141.

Blomstróm, M. and F. Sjöholm (1999). "Technology transfer and spillovers: Does local participation with multinationals matter?" Exropean Esonownic Revtew, 43: $915-923$.

Bloom, N. and J. V. Reenen (2000). "Patents, productivity and market value in a panal of British firms." London, Tbe Inshitute for Fiscal Stadier 27.

Bower, G. H. and E. R. Hilgard (1981). Tbeonies of haming. Englewood Cliffs N], Prentice-Hall.

Brass, D.J., K.D. Butterfield, et al. (1998). "Relationships and unethical behavior: a social network perspective." Aradewy of Managemo Rowa, 23(1):14-31.

Bresch, S. (2000). "The geography of innovation: a cross-industry analysis". Regional Sindies, 34: $213-229$.

Breschi, S., F. Malerba, et al. (2000). "Technological regimes and Schumpeterian patterus of innovation." Econamic Jowmal, 110:388-410.

Burt, $\mathbb{R}$ S. (1992). Stmotwh Holes. The social stmotwe of tompetition. Cambridge M.A., Harvard University Ptess.

Cameron, A. C. and P. K. "Trivedi (1986). "Econometric models based on count data: Comparisons and applications of some estimators and tests." Jommal of Applied Eowownervits, $1: 29-53$. 


\section{REFERENCES}

Cameron, A. C. and P. K. Trivedi (1990). "Regression-based tests for overdispersion in the Poisson modiel." Jownal of Economemes, 46: $347-364$.

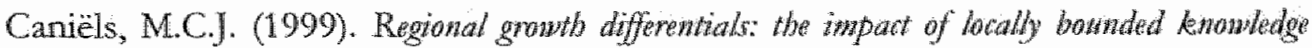
spillowers. Maastricht University Press. Maastricht.

Cantwell, J. (1991). Historical trends in intemational pattems of technological

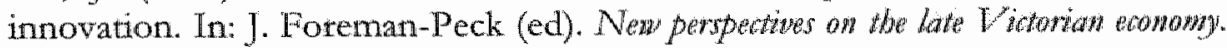
Cambridge, Cambridge University Press.

Cantwell, J. and O. Janne (1999). "Technological globalisation and innovative centres: the role of corporate technological leadership and locational hietarchy." Research Palio, 29: 119-144.

Cantwell, J. and S. Iammarino (2001). "EU regions and multinational comporations: change, stability and strengthening of technological comparative advantages." Indworial and Corporate Change, 10(4): 1007-1037.

Cantwell, J. and S. Iammarino (2003). Multinational corporations and Europeaw regional systems of inmonation. London, Routledge.

Capron, L. (1999). "The long-term performance of hotizontal acquisitions." Strategrc Managewent Jownal, 20: 987-1018.

Carpenter, M. P., F. Narin, et al. (1981). "Citation rates to technologically important patents." World Patent Information, 3(4): $160 m 163$.

Carpenter, M. P. and F. Narin (1983). "Validation study: Patent citations as indicators of science and foreign dependence." Whorld Palent Information, 5(3): 180-185.

Cohen, M. D. (1991). "Individual leatning and organizational routine: emerging connections." Onganization Saence, 2(1): 135-139.

Cohen, W. M. and D. A. Levinthal (1989). "Innovation and learning: The two faces of R\&D." Tbe Economic Jommal, 99 (September): $569-596$.

Cohen, W. M. and D. A. Levinthal (1990). "Absorptive capacity: A new perspective on learning and innovation." Adminisfative Scieve Qmarterb, 35: $128-152$. 


\section{REFERENCFS}

Cohen, W. M. and S. Klepper (1996). "A reprise of size and R\&D." The Economic Journal, 106 (July): $925-951$.

Cohen, W. M., A. Goto, et al. (2002). "R\&D spillovers, paterits and the incentives to innovate in Japan and the United States." Ressearch Poling, 31: 1349-1367.

Conner, K. R. (1991). "A historical comparison of tesource-based theory and five schools of thought within industrial organization economics: do we have a new theory of the firm?" Journal of Management, 17(1): 121-154.

Cook, C. (2002). Patents, profits and power. London, Kogan Page Limited.

Cowan, R., P. A. David, et al. (2000). "The explicit economics of knowwledge codification and tacitness." Industrial and Conporate Change, 9(2): 211-253.

Criscuolo, P., R. Narula, et al. (2001). Measuring knowledge flows among European and American multinationals: a patent citation analysis. Paper propared for the Ecis conference on the future of innowation studies, Eindhoven, the Netherlands.

Cyert, R. and J.G. March (1963). A behavioural theory of the from, Englewood Cliffs, Prentice-Hall.

Dierickx, I. and K. Cool (1989). "Asset stock accumulation and sustainability of competitive advantage." Managemen Science, 35: 1504-1511.

Dodgson, M., (ed.) (1989). Technology Strategy and the farm: managemsent and public poligy. Essex, Longman Group UK Iimited.

Dollinger, M. J, P. A. Golden, et al. (1997). "The effect of teputation on decision to joint venture." Stratigi Managenzent Joumal, 18(2): 127-140.

Doz, Y. L. and G. Hamel (1998). Alliance advantage. Boston MA, Havard Business School Press.

Drejer, A. (2000). "Organisational learning and competence development." The Lataning Oryanization, 7(4): 206-220.

Dumont, M. and $A$. Tsakanikas (2001). Knowledge spillovers through R\&D networking. CESIT Discussion Paper: 22.

Dunning, J. and R. Narula (1995). "The R\&D activities of foreign firms in the United States." International Studies of Managentent \& Organization, 25: 39-73. 
Duysters, G. and J. Hagedoom (1995). "Strategic groups and Inter-firm networks in international high-tech industries." Joumal of Management Studier, 32(3): 359-381.

Duysters, G., G. Kok, et al. (1999). "Crafting successful strategic technology partnerships." ReoD Management, 29(4): 343-351.

Duysters, G. and A. D. Man (2002). Samenwerking en innovatie. Literatuuroverzicht van de relatie tussen innovatiekracht en interorganisatorische samenwerking. EZ onderzoekesreeks. Den Haag. 03107.

Duysters, G. and Ch. Lemmens (2003). "Alliance group formation: enabling and constraining effects of embeddedness and social capital in strategic technology alliance networks." International Studies of Management and Organization, 33(2): 49. 68.

Duysters, G., J. Hagedoorn et al. (2003). "The innovative performance of alliance block members in changing technological environments: evidence from the microelectronics industry". Unpublished paper.

Dyer, J. $\mathbb{H}$. and $\mathbb{H}$. Singh (1998). "The relational view: cooperative strategy and sources of interorganizational competitive advantage." Academy of Managenent Review 23(4): 660-679.

Edmondson, A. C., R. M. Bohmer, et al. (2001). "Disrupted routines: Team learning and new technology implementation in hospitals." Administrative Science Quarterly, 46: 685-716.

Foss, N. J. (1996). "Knowledge-based approaches to the theory of the firm: some critical comments." Organization Seintee, 7(5): 470-476.

Fourastié, J. Le grande espoir du XXe siècle: progrès technique, ptogrès ćconomique, Progrès social, Paris, 1949. In: J. Fourastié: Machinisme et bientaếtre, Pátis, 1951, édition de minuit. Presses Universitaires de France.

Freeman, C. and L. Soete (1997). The economicr of industrial innowation. London, Pinter.

Friedkin, N.E. (1984). "Structural cohesion and equivalence explanations of social homogeneity." Saciological Metbods and Research, 12: 235-261.

Gertler, M. (2001). Tacit knowledge and the economic geography of context. Paper prepared for the DRUID sumwer conference, Aalborg, Denmark. 
Gilbert, M. and M. Cordey-Hayes (1996). "Understanding the process of knowledge transfer to achieve successful technological innovation." Telbnovation, 16(6): 301.312.

Granovetter, M. S. (1973). "The strength of wealk ties." Anwerican Journal of Socialogy $78(6): 1360-1380$.

Granovetter, M. S. (1992). Problems of explanation in economic sociology. In: N. Nohria and R.G. Eccles (eds.), Networles and organizations: structure, form and action. Boston, Harvard business School Press: 25-56.

Grant, R. M. and C. Baden-Fuller (1995). "A Knowledge-based theory of interfirm collaboration." Proceedings of the Academy of Management Conference: 17-21.

Grant, R. M. (1996). "Toward a knowledge-based theory of the firm." Strategir Management Journat, 17(Winter special issue): 109-122.

Greene, W. H. (2000). Econometric analysis. New Jersey, Prentice-Hall.

Griliches, Z., (ed.) (1984). RœD, Patents, and Productivity. Chicago, The University of Chicago Press.

Griliches, Z. (1990). "Patent staristics as economic indicators: A survey." Joumal of Economic Literature, 28(4): 1661-1707.

Griliches, Z. (1998). RevD and Prodwotivity - The econometric evidente. Chicago, The University of Chicago Press.

Gulati, R. (1995). "Does familiarity breed trust? The implications of repeated ties for contractual choice in alliances." Aademy of Management Journal, 38(1): 85-112.

Gulati, R. (1998). "Alliances and networks." Strategic Managensent Jostral, 19: 293-317.

Gulati, R. and M. Gargiulo (1999). "Where do interorganizational networks come from?" Anserican jommal of Soriology, 104(5) March: 1439-93.

Gupta, A. K. and V. Govindarajan (2000). "Knowledge flows within multinational corporations." Strategic Management Journal, 21: 473-496. 


\section{REFERENCES}

Hagedoorn, J. and J. Schakentaad (1990). Inter-firm partnerships and co-operative strategies in core technologies. In: C. Freeman and L. Soete (eds.), Nen explorations in the econownics of tedmical change. London, Frances Pinter: 3-37.

Hagedoorn, J. (1993a). Strategic technology alliances and modes of cooperation in high-technology industries. In: G. Grabher (ed.), The enbedded firm, on the socioeconomics of industrial metavortes. London, Routledge: 116-137.

Hagedootn, J. (1993b). "Understanding the rationale of strategic technology partnering: Interorganizational modes of cooperation and sectoral differences." Strategic Managennen Journal, 14(5): 371-385.

Hagedoorn, J. and J. Schakenraad (1994). "The effect of strategic technology alliances on company performance." Strategir Management Journal, 15: 291 -309.

Hagedoorn, J. (1995). "A note on international market leaders and networks of strategic technology partnering." Strategic Management Jourmal, 16: 241-250.

Hagedoorn, J. and G. Duysters (1999). External appropriation of inwovative capabilities: the preference for strategic alliances or Mos. As: 36.

Hagedoorn, J. (2002). "Inter-firm R\&D partnerships: an overview of major trends and patterns since 1960." Research Policy, 31: 477-492.

Hagedoom, J. and G. Duysters (2002). "External sources of innovative capabilities: the preference for strategic alliances or mergers and acquisitions." Journal of Managernent Studies, 39(2): 167 188.

Hagedoom, J. and M. Cloodt (2003). "Measuring innovative performance: is there an advantage in using multiple indicators?" Research Polig, 32(8): 1365-1379.

Hägerstrand, T. (1967). Innovation diffuston as a spatial process. Chicago.

Hall, B., A. B. Jaffe, et al. (2001). The NBER patent citations data file: lessons, insights and methodological tools. NBER Working Paper Series. Cambridge MA: 74.

Hamel, G. (1991). "Competition for competence and inter-partner learning within international strategic alliances." Strategic Management Jourmal, 12: 83-103.

Hausman, J., B. H. Hall, et al. (1984). "Econometric models for count data with an application to the patent-R\&D relationship." Econometrica, 52(4): 909-938. 


\section{REFERENCES}

Fospers, G.-J. (2003). "Beyond the Blue Banana? Structural change in Europe's geoeconomy." Intrecomontos, (March/April): 76-85.

Inkpen, A. C. (1998). "Learning and knowledge acquisition through international stranegic alliances." The Acadwy of Maragement Executine, 12(4):69-80.

Inkpen, A. C. and A. Dinur (1998). "Knowledge management processes and international joint ventutes." Organzation Schence, 9(4): 454-468.

Inkpen, A. C. (2002). Learning, knowledge management, and strategic alliances: so many studies, so many unanswered questions. In: F. Contractor and $P$. Lorange (eds.), Cooperatiwe strategies and allantes. Amsterdam, Pergamon. 267-289.

Jaffe, A. B., M. Trajtenberg, et al. (1993). "Geographic localization of knowledge spillovers as evidence by patent citations." The Quarterly Jawnal of Economics, 108 : $577-598$.

Jaffe, A. B. and M. Trajtenberg (1996). Flows of knowledge from universities and fedetal labs: Modelling the flow of patent citations over time and actoss institutional and geographic boundaries. NBER Working Paper Serien. Cambridge MA: 30.

Jaffe, A. and M. Trajtenberg (1998). International Knowledge Flows: Evidence from patent citations. NBER WFonking paper Series. Cambridge MA, National Bureau of Economic Research: 39.

Jaffe, A. B., M. S. Fogarty, et al. (1998). "Evidence from patents and patent citations on the impact of NASA and other tederal labs on commercial innovation." Tbe Jownal of Industral Exonomics, 46(2): 183-205.

Jaffe, A. B., M. Trajtenberg, et al. (2000a). "Knowledge spillovers and patent citations: Evidence from a survey of inventors." Amorican Economic Review, Papers and Procedings: $215-218$.

Jaffe, A. B., M. Trajtenberg, et al. (2000b). The meaning of patent citations: Report on the NBER/Case-westem teserve survey of patentees. NBER Working Paper Serros. Cambridge MA: 43.

fafte, $\mathrm{A}_{n} \mathrm{~B}$, and M. Trajtenberg (2002). Patents, citations and innowations - a whdow on the knowledge enonony. Cambridge MA, The MIT Press. 


\section{REFERENCES}

Johnson, B., E. Lorenz, et al. (2002). "Why all this fuss about codified and tacit knowledge?" Industial and Coponate Change, 11:245-262.

Kamien, M. I. and N. L. Schwartz (1982). Manhet strutwe and innowation. Cambridge, Cambridge University Press.

Khanna, T. (1998). "The scope of alliances." Orgawnotion Sowne 9(3): 340-355.

Khanna, T., R. Gulat, et al. (1998). "The dynamics of learning alliances: Competition, cooperation and relative scope." Strategic Matagenwew Jowmal, 19(3): $193-210$.

Kogut, B. (1988). "Joint ventures: Theoretical and empirical perspectives." Strategic Managoment Jommal, 9: 319-332.

Kogut, B. and U. Zander (1992). "Knowledge of the firm, combinative capabilities, and the replication of technology." Organization Saence, 3(3): 383-397.

Krackhardt, D. (1992). "The strength of strong ties: the importance of philos in organizations. In: Nohria and Eccles (eds.), Nutworks and organizations: strwotur, farm and action: $216-239$.

Knugman, P. (1991). Geograpby and trade. Cambridge, MA, MrT Press.

Lane, P. I. and M. Jubatkin (1998). "Relative absorptive capacity and interorganizational leaming." Sirategic Managentent Joumal, 19: 461-477.

Lane, P. J. J. E. Slak, et al. (2001). "Absorptive capacity, learning, and performance in international joint ventures." Stratogic Management Joumal, 22: 1139-1161.

Larsson, R., L. Bengtsson, et al. (1998). "The interotganizational learning dilenma: collective knowledge development in strategic alliances." Organtration Sience, $9(3): 285-305$

Le Bas, C. B. and C. Sietra (2002). "Location versus home country advantages in R\&D activities: some further tesults on multinationals locational strategies." Rerearch Policy, 31: 589-609.

Leonard-Barton, D. (1995). Wellsprings of knowledge. Boston, Harvard Business School Press. 


\section{REHERENCES}

Levin, R C., A. K. Klevorick, et al. (1987). "Appropriating the teturns from industrial research and development." Brooking papers on economic activity: 783-831.

Levinthal; D. A. (1994). Surviving Schumpeterian environments: an evolutionary perspective. In: J. A. C. Baum and J. V. Singh (eds.), Evolutionary dynamizes of arganizationis. New York, Oxford University Press.

Levitt, B. and J. R. March (1988). "Organizational learning." Anntal Revziew of Sociology, 14: $319-340$.

Lucas, R. E. (1988). "On the mechanics of economic development." Journal of Monetary Ecomomics, 22: 3-42.

Mansfield, E. (1986). "Patents and innovation: an empirical study." Management Srience, 32: 173-181.

Marshall, A. (1936). Principles of economics. London, MacMillan and Co. Ltd.

Marsili, O. (2001). The anatomy and evolution of industries" technological change and industrial dymaniss. Cheltenham, Edward Elgar.

Marx, K. (1858). Grundrisse. London, Allen Lane (1973).

Maurseth, P. and B. Verspagen (2002). "Knowledge spillovers in Europe. A patent citation analysis." Scandinavian Jownal of Economics, 104: 531-545.

Meyer, M. (2002). "Tracing knowledge flows in innovation systems." Scientometrics, 54(2): 193-212.

Michel, J. and B. Bettels (2001). "Patent citation analysis. A closer look at the basic inpur data from patent search teports." Satentometrics, 51(1): 185-201.

Mody, A. (1993). "Learning through alliances." Journal of Economic Bebarior and Organization, 20: 151-170.

Monjon, S. and P. Waelbroeck (2003). The nature of innovation and the origin of technological spillovers: an econometric analysis on individual French data. Minno: 26.

Morgan, K. (2004). "The exaggerated death of geography: learning, proximity and territorial innovation systems." Journal of Economic Geography, 4: 3-21. 


\section{REFERENCES}

Mowery, D. C. (1983). "The relationship between intrafirm and contractual forms of industrial research in American manufacturing, 1900-1940." Explonations in Econownic History, 20: 351-374.

Mowery, D. C., J. E. Oxley, et al. (1996). "Strategic alliances and interfirm knowledge transfer." Strategic Managment Jotrmal, 17 (Winter special issue): 77-91.

Mowery, D. C., J. E. Oxley, et al. (2002). The two faces of partner-specific absorptive capacity: learning and cospecialization in strategic alliances. In: F. Contractor and P. Lorange (eds.), Cooperative strategies and alliames. Amsterdam, Pergamon. $291-319$.

Mueller, D. C. (1986). The modern conporation. Brighton, John Spiers.

Mytelka, L. K., Ed. (1991). Strategic Parnerships. London, Pinter Publishers Limited.

Nadiri, M. I. (1993). "Thnovations and technological spilloyers." Econowic Research Reports. New York: 47.

Nakamura, M., J. M. Shaver, et al. (1996). "An empirical investigation of joint venture dynamics: Evidence from U.S.-Japan joint ventures." International jourmal of Industrial Organization 14:521-541.

Napolitano, G. and G. Sirilli (1990). "The patent system and the exploitation of inventions: Results of a statistical survey conducted in Italy." Technovation, 10(1): 5-16.

Narin, F., E. Noma, et al. (1987). "Patents as indicators of corporate technological strength." Research Poliy, 16: 143-155.

Nelson, R. and S. Winter (1982). An evolutionary theory of economic change. Cambridge, Harvard university press.

Nelson, R.. R. (1991). "Why do firms differ, and how does it matter?" Strategir Management Joumal, 12 (Winter Special Issue): 61-74.

Niosi, J. (1999). "The internationalization of industrial R\&D. From technology transfer to the learning organization." Research Policy, 28: 107-117.

Nonaka, T., P. Byosiere, et al. (1994). "Organizational knowledge creation theory: a first comprehensive test." International Basiness Remien, 3(4): 337-351. 


\section{RETERENCES}

Paci, R. and S. Usai (2000): "Technological enclaves and industrial districts: an analysis of the regional distribution of innovative activity in Europe." Regional Sindies, 34(2): 97-114.

Paci, R. and S. Usait (2001). "Externalities, knowledge spillowers and the spatial distribution of innovation:" GEOjournal, 52(12).

Pakes, A. and Z. Griliches (1980). Patents and R\&D at the firm level: a first look. NBER Wrorking Paper Series. Cambridge MA: 33.

Parise, S. and J. C. Henderson (2001). "Knowledge resource exchange in strategic alliances." IBM Systems Journal 40(4): 908-924.

Parker, J. E. S. (1978). The economics of inmonation. The national and muthinational enterprise in technological change. London, Longman.

Parkhe, A. (1991). "Interfirm diversity, organizational learning, and longevity in global strategic alliances." Journal of International Business Studies, 22(4): 579-601.

Patel, P. and K. Pavitt (1991). "Large firms in the production of the world's technology: An important case of "non-globalisation"." Journal of Intermational Business Siudies, First Qwarter: 1-21.

Patel, P. and K. Pavitt (1997). "The technological competencies of the world's largest firms: Complex and path-dependent, but not much variety." Research Poligy, 26: $141-156$.

Patel, P. and M. Vega (1999). "Patterns of internationalisation and corporate technology: location versus home country advantages." Reseamb Polig, 28: 145155.

Patel, P. (2000). Technological Indicators of Performance. In: J. Tidd (ed.), From Knowledge Management to Strategic Competence: Measuring Tedbrological, Market and Organisattonal Intovation. London, Imperial College Press. 3.

Pavitt, $\mathbb{K}$. (1984). "Sectotal patterns of technical change: towards a taxonomy and a theory." Researd Poligy, 13: 343-373.

Pavitt, K. (1988). Uses and abuses of patent statistics. Handbook of quantitative studies of science and technology. In: A. F. J. v. Raan (ed.), Handbook of quanitative studies of science and lecbnology. Amsterdam, Elsevier: 509-536. 


\section{RETERENCES}

Penrose, E. T. (1959). The theory of the gronth of thie from. London, Basil Blackwell Publisher.

Peteraf, M. A. (1993). "The comerstones of competivive advantage: a resource-based wiew." Strategic Management Jown al, 14(3): 179-191.

Plasmans, J. and R. Lukach (2001). Measuring knowledge spillovers using Belgium EPO and USPTO patent data. CESFf Wrorteing paper. Munich: 21.

Powell, W. W., K. W. Koput, et al. (1996). "Interorganizational collaboration and the locus of innovation: Networks of learning in Biotechnology." Admimistrative Science Quarterly, 41(March): 116-145.

Prahalad, C. K. and G. Hamel (1990). "The core competence of the corporation." Hanard Busimess Review, May-June: 79-91.

Romer, P. M. (1986). "Increasing returns and long-run growth." Joumial of Politiral Eronomy, 94(5): 1002-1037.

Romer, P. M. (1990). "Endogenous technological change." Jormal of Political Economy, 98: $\$ 71-S 102$.

Rumelt, R. P. (1996). Towards a strategic theory of the firm. Resources, firms, and strategies. In: N. J. Foss. (ed.) Resourres, fimms, and strategies. Oxford, Oxford University Press: 131-145.

Sarkar, M. P., R. Echambadi, et al. (2001). "Alliance entrepreneurship and firm market performance." Strategir Managentent Journal, 22: 701-711.

Saxenian, A. (1994). Regional advanage. Cuiture and antetition in Silicon Valley and Route 128. Cambridge MA, Harvard University Press.

Schätzl, L. (ed.): Wirschaftrgeographie der Europäuschen Genneinschaft, Stuttgart, 1993. Uni-TB.

Schein, E. H. (1996). "Three cultures of management: the key to otganizational learning." Sloan Managentent Review, 38(Fall): 9-20.

Scherer, F. M. (1965). "Firm size, market structure, opportunity, and the output of patented inventions." The American Economic Review, 55(5): 1097-1125.

Scherer, F. M. (1984). Innowation and Growth. Cambridge MA, The MIT Press. 


\section{REHERENCES}

Schumpeter, J. A. (1934). The theory of economic development. Cambridge, Harvard university press.

Schumpeter, J. (1939). Business goles: a therrtind bistoncal and statistical analysis of the capitalist process. New York, McGraw-Hill.

Schumpeter, J. A. (1942). Capitalism, Socialism and Democray. New York, Harper \& Row.

Smith. A. (1776). An inquiry into the mature and catuses of the whalth of nations, Penguin (1999).

Steenhuis, H.-J. and E. J. De Bruijn (2002). "Technology transfer and learning." Toronology Analysis and Strategic Management, 14(1): 57-66.

Stel, A. v. and H. Nieuwenhuijsen (2002). Knowledge spillowers and economir growth: an anpwrial awalysis for the Nothenands. Discussion paper. Rotterdam: 29.

Stuatt, T. E. and J. M. Podolny (1996). "Local seatch and the evolution of technological capabilities." Strategic Managentent Journal, 17:21-38.

Stuart, T. F. and J. M. Podolny (2000). Positional causes and consequences of alliance formation in the semiconductor industry. In: J. Weesie and W. Raub (eds.) The mandgentent of dwable relations: theoretical models and empincal studies of bouselolds and organizations. Amsterdam, Thelathesis.

Szulanski, G. (1996). "Exploring intenal stickiness: impediments to the transfer of best puactice within the firm." Strategh Mamagement Jommal, 17(Winter Special $(\operatorname{sinuc}): 27-43$.

Tecce, D. J. (1986). "Profiting from technological innovation: Implications for" integration, collaboration, licensing and public policy." Researd Polig, 15: 285305.

Teece, D. J., G. Pisano, et al. (1997). "Dynamic capabilities and strategic management." Strategic Managew*nt Jourmal 18(7): 509-533.

Trott, P., M. Cordey-Hayes, et al. (1995). "Inwatd technology transfer as an interactive process." Tedhowarion, 15(1): 25-43. 


\section{REFERENCES}

Uzzi, B. (1997). "Social stmcture and competion in intetfirm networks: the paradox

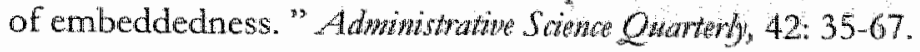

Vanhaverbeke, W., G. Duysters, et al. (2002). "A longitudinal analysis of choice between technology based strategic alliances and acquisitions in high-tech industries: the case of the ASIC industry." Onganization Shent, 13(6): 714-733.

Verspagen, B. (1996). Technology indicators and econonic growth in the European area: Some empirical evidence. In: B. V. Ark and N. Crafts (eds.), Comparative Experience of Economic Growt in Pastor Enope. Cambridge, Cambridge UP: 215 . 243.

Verspagen, B. and W. Schoenmakers (2004). "The spatial dimension of patenting by multinational firms in Eutope." Jounnal of Economuc Geograpby, 4(1): 23-42.

Von Hippel, E. (1994). "Sticky information and the locus of problem solving: implications for inthovation." Mangewent Sciene, 40(4):429-439.

Wemerfelt, B. (1984). "A resource-based view of the firm." Strategic Mawaymswn" Jowring, 5(2): 171-180.

Westney, D. E. (1988). Domestic and foreign leaming curves in managing international cooperative strategies. In: F. Contractor and P. Lotange (cds.) Coopenative strategies in intenational bxsiness. Lexington, MA. Lexington Books. 339-346.

Zahra, S. and G. George (2002). "Absorptive capacity: a review, reconceptualization and extension." Aradewy of Management Reviow, 27 (2): 185-203. 



\title{
Nederlandse Samenvatting Kennisstromen tussen Multinationale Ondernemingen.
}

\author{
Een Patent Data Analyse
}

\section{Introductie.}

Dit proefschrift bestudeert de eigenschappen van de kennisstromen die optreden tussen multinationale ondernemingen die actief zijn binnen de hoogen middeltechnologische sectoren van de economie. Uit de litemtuur weten we dat kennisstromen uitermate belangrijk zijn voor ondernemingen, omdat ze belangrijk zijn voor het leren binnen ondernemingen, wat weer belangrijk is voor de bedrijfsresultaten van de ondememing. Om een indruk te krijgen van de manier waarop bedrijwen van elkaar leren is het dus belangrijk de aard wan de kennisstromen tussen bedrijven te onderzoeken. De centrale probleemstelling van dit proefschrift luidt dan ook: wat zijn de eigenschappen nan de kennisstromen thassem mulinationale ondernemingen? Deze centsale probleemstelling kan worden opgedeeld in drie deelvragen. Allereerst, welke factoren beinvloeden de kennisstromen tussen multinationale ondernemingen en wat is de richting van hun beinvloeding? Voorts, welke invloed hebben strategische technologie allianties op de kennisstromen tussen multinationale ondernemingen? En tenslotte, zijn kennisstromen ruimrelijk gebonden?

Om dit onderzock te kunnen uitvoeten hebben we allereerst een uitgebreide eigen databank gebouwd (zie hoofdstuk 2). Deze databank combineert de informatie van meerdere bestaande databanken, maar voegt tevens voor een belangrijk deel extra informatie toe. De belangrijkste gebruikte databanken zijn de European Patent Office (EPO) databank met informatie over bijna 1 miljoen patenten en de MERIT-Cooperative Agreements and Technology Indicators (CATI) databank die informatie bevat met betrekking tot meer dan 15.000 samenwerkingsverbanden tussen meer dan 9.500 bedrijyen. De basis voor onze databank vormt de Fortune 500 lijst met de 500 grootste bedrijven ter wereld. Uit deze lijst hebben we die bedrijven gekozen die werkzaam zijn binnen hoog- en middeltechnologische sectoren. Uiteindelijk hielden we 171 multinationale moederbedrijven over. Vervolgens hebben we gebruik gemaakt van de Dun \& Braclstreet linkages databank, waarin alle bedrijfsstambomen staan van leder moederbedrijf met alle 
dochterondernemingen. Middels deze databank hebben we alle patenten die we konden vinden in de EPO databank van de dochterbedrijven en het moederbedrijf geaggregeerd tot op moederniveau. Deze uitgebreide databank vormt de basis wan het onderzoek in dit proefschtift.

\section{Theoretisch achtergronden}

Reeds Adam Smith (1776) erkende het belang van innovaties zowel voor de groei van de economie in zijn geheel als voor de groei van de individuele onderneming. Ook Karl Marx (1858) zag uitvindingen en innovaties als zeer belangrijke elementen voor de groei van de economie. Het was Joseph Schumpeter (1934; 1939 en 1942) die de grondbeginselen legde voor de hedendaagse economische theorieën met betrekking tot innovatie. Hij beschreef de invloed van innovaties op de economie op basis van twee concepten, "creaticve vetnietiging" en "creatieve accumulatie". In de tachtiger jaren van de worige eeuw ontstond de "New Growth theory", vooral op basis van werk van Romer (1986 en 1990) en Lucas (1988). In deze theorie krijgt technologische verandering een zeer centrale plaats die zorgt voor voordelen voor zowel de individuele onderneming als voor de economie in zijn geheel.

Wat alle vootgaande theorieen gemeen hebben is dat ze zich rootal concentreren op de invloed van kennisstromen op de economie in zijn geheel. Hoewel de invloed van kennisstromen op de economie als geheel uiterst belangrijk is, zijn wij, woor ons onderzoek, meer geïnteresseerd in de invloed van kennisstromen op de individuele onderneming. We dienen ons derhalve te tichten op de theorieën van de ondememing. Een van de invloedrijkste theorieën van de onderneming is de "resource-based" theorie, die zijn oorsprong vindt in het werk van Penrose (1959) en Wernerfelt (1984). Deze theorie verklaart verschillen tussen ondernemingen op basis van de mate waarin de ondernemingen strategische huipbronnen beheren en veronderstelt dat deze verdeling van hulpbronnen vrij stabiel is. De bedrijfshulpbronnen worden gezien als waardevol, zeldzaam en lastig te imiteren of te substitueren. Vandaar dar bedrijven die deze hulpbronnen beheren economisch beter presteren dan bedrijven die deze hulpbronnen ontberen. Voortbouwend op deze theorie ontstond de "dynamic capabilities" theorie (Teece, Pisano et al 1997). Ook deze theorie stoelt op de veronderstelling dat bedrijfshulpbronnen wan belang zijn voor de bedtijfsresultaten van een onderneming. In tegenstelling tot de "resource-based" theorie gaat de "dynamic capabilities" theorie er van uit dat ondernemingen nieuwe hulpbronnen kunnen verkrijgen, onder andere door middel van organisationeel leren. Een derde theorie die hier van belang is, is de "knowledge-based" theorie. Ook deze theorie baseert zich op de "resourcebased" theorie en onderschrijtt het belang van organisationeel leren. In deze 
theorie is de belangrijkste hulpbron binnen het bedrijf de kennis die in de onderneming opgeslagen is. Het betreft hier zowel de kennis rervat in bijvoorbeeld patenten, als ook de kennis die anwwezig is bij de individuele wetknemers of binnen de bedriffstoutines.

\section{Factoren die de kennisstromen beïnvloeden.}

Als eerste onderdeel van de beantwoording van de centrale probleemstelling hebben we gekeken naar de factoren die de kennisstromen tussen multinationals beïnvloeden (zie hoofdstuk 3). Op basis wan literatuuronderzoek werd een aantal factoren geidentificeerd. De belangrijkste factoren blijken het absorberend vermogen van de onderneming, de relatieve hoogte van de uitgaven voor onderzoek en ontwikkeling $(O \& O)$ en het aantal samenwerkingsverbanden van het bedrijf met andere bedrijwen: het aantal allianties. Middels onze eigen databank waren we in de gelegenheid deze theoretisch veronderstelde verbanden te onderzoeken en tevens de tichting van de verbanden vast te stellen. Middels negatief binomiale regressie hebben we de verbanden onderzocht.

Voor de operationalisering van onze variabelen hebben wij gebruik gemaakt van de definities van Jaffe en Trajtenberg (1998) die een patent een maat noemen voor een stukje kennis, en een patent citaat een maat voor een kennisstroom. Het absorberend vermogen van de onderneming, gedefinieerd als het totaal aantal patenten van de onderneming blijkt een positieve invloed te hebben op de kennisstromen, zowel wan andere bedrijven naar het bedrijf toe, als andersom. Bedrijven met meer patenten hebben meer kennis in huis om kennis van buiten op te nemen. Anderzijds zijn ze door hun grotere aantal patenten interessanter voor andere bedrijven om kennis te vergaten. De tweede factor, de relatieve uitgaven aan $O \& O$, heeft ook een positieve invloed op de kennisstromen, zowel naat het bedrijf toe als omgekeerd. De verklatingen zijn vrijwel identiek aan die voor het absorberend vermogen. De derde en lateste onderzochte factor, het aantal allianties, blijkt eveneens een positieve invloed te hebben op de kennisstromen en weer in beide richtingen. Verder blijkt de grootte van het bedrijf belangrijk voor de mate waarin kennis naar het bedrijf toestroomt. Over het verband tussen de mate waarin kennis van het bedrijf weg stroomt en de bedrijfsgrootte valt, op basis van ons onderzoek, niks te zeggen.

De drie onderzochte factoren blijken dus een positieve invloed te hebben op de kennisstromen zowel naar het bedrijf toe als van het bedrijf af. 


\section{Leren binnen strategische technologie allianties.}

$\mathrm{Na}$ de factoren vastgesteld te hebben die een invloed hebben op de kennisstromen tussen multinationale ondernemingen wilden we vervolgens één van deze factoren verder gaan uitdiepen. We wilden met name kijken naar het leren binnen strategische technologie allianties (hoofdstuk 4). Strategische technologie allianties worden steeds vaker aangegaan met de bedoeling van elkate teren, wat uiteraard leidt tot kennisstromen tussen de bedrijven in een alliantie. Om te beginnen hebben we de relatie onderzocht van het leten binnen strategische technologie allianties. Wij veronderstellen daarbij een negatief parabolisch verband tussen het leren binnen de alliantie en de kennisbasis overlap van de bedrijven in de alliantie. De kennisbasis van een bedrijf hebben we geclefinieerd als de patenten van een bedrijf plus de patenten (van andere bedrijven) waar de bedrijven naar verwijzen in hun eigen patenten, de zogenaamde patent citaten. Voor de operationalisering van onze variabelen hebben wij wederom gebruik gemaakt van de definities van Jaffe en Trajtenberg (1998). We verwachtten dat het leren eerst toeneemt naarmate de overlapping in kennisbases toeneemt, maar dat deze na een bepaald omslagpunt weer afneemt. Verwolgens hebben we verondersteld dat er meer geleerd zal worden binnen allianties die zijn opgezet met het doel van elkaar te leren dan wanneer het niet zulke allianties betreft. Om deze veronderstelling te testen hebben we een controle groep moeten bouwen aangezien we alleen gegevens van leerallianties hebben. Als laatste wilden we de invloed van de sterkte van de verbanden binnen de allianties vergelijken met de kennisoverdracht tussen de bedrijven. Onze veronderstelling was dat bedrijven met een sterk alliantie werband meer van elkaar zouden leren dan bedrijven die via een zwak verband met elkaar verbonden zijn.

Om een en ander te testen hebben we wederom gebruik gemaakt van onze eigen databank. Ten gevolge van de beperkingen van de voorhanden zijnde gegevens dienen we gebruik te maken van Tobit regressie. Onze eerste twee hypotheses werden door onze analyses bevestigd. Leren binnen allianties gebeurt dus in de vorm van een negatief parabolisch verband met betrekking tor de yoor-alliantie kennisbasis overlapping. Bedrijven leren eerst meer van elkaar, waarna een maximum bereikt wordt en de bedrijven vervolgens minder van elkaar leren. Ook blijken zogenaamde leer-allianties uitermate effectief voor kennisoverdracht tussen de alliantie partners. Tot onze verbazing blijkt echter onze derde hypothese niet bevestigd te worden door onze analyses. Blijkbaar zijin zwakke verbindingen tussen de bedrijven in onze databank belangrijker voor kennisoverdracht dan sterke verbindingen. Vertrouwen aussen de samenwerkende bedrijven is blijkbaar minder belangrijk dan complementariteit. Verder is het mogelijk dat de onderzochte bedrijven lijden aan zogenaamde "over-embeddedness". Doordat bedrijven te sterk vertrouwen op enkele 
alliantie partners waar ze sterke verbanden mee hebben leren ze uiteindelijk minder van deze partners. Ze weten het meeste immers al rengevolge van de voorgaande samenwetkingsverbanden.

\section{De ruimtelijke dimensie van patentering door multinationale bedrijiven in Europa.}

Als laatste hebben we onderzoek gedaan naar de ruimtelijke dimensies van de kennisstromen van multinationale ondernemingen. Daaronder wordt allereerst verstaan dat gekeken wordt naar de geografische verdeling wan de O\&O activiteiten van de bedrijven in onze databank binnen Eutopa. We zijn dus geïnteresseet in hoeveel $O \& O$ activiteiten de bedrijven verrichten binnen en buiten hun eigen regio. Concentreren deze activiteiten zich in bepaalde regio's? En als dit zo is, in welke regio's treedt deze concentratie dan op? Dit zijn de vrager die we in het eerste gedeclte van hoofdstuk 5 wilden beantwoorden. Vervolgens hebben we gekeken naar de verspreiding van kennis in de tijd en over regio's. Opnieuw hebben we gebruik gemaakt van onze eigen databank. We hebben allereerst gekeken naar de O\&O activiteiten van de bedrijven in onze databank en met name naar de spreiding hiervan over de NUTS regio's binnen Europa. De patenten worden daarbij toegewezen aan de regio door middel van de postcode van de uitwinders. Overigens is dit de eerste keer dat dit soort onderzoek platsvindt op deze schaal en met deze mate van specificiteit voor heel Europa. Het blijkt dat een redelijk deel van de O\&O activiteiten buiten de eigen regio wan de onderneming plaatsvindt. Voorts blijken slechts een beperkt aantal regio's het leeuwendeel van deze O\&O activiteiten te ontvangen. Met name zijn dit drie regio's in Duitsland, het gebied rond Londen en dat rond Parijs.

Onze resultaten vallen samen met ander onderzock (m.n. Hospers 2003) waarbij binnen Europa een bepaald kerngebied op het vlak van innovatie wordt aangewezen. Dit zogenaamde "Blue Banana" gebied, zo genoemd naar de vorm en de kleur van de regio bij de eerste presentatic, loopt grofweg van Londen via de Benelux en de Rijntegio naar Milaan. Dit gebied herbergt van oudsher belangrijke innovatieve centra. Het blijkt dan ook dat de verdeling van innovatieve activiteiten over regio's viij stabiel is over tijd. Achtergestelde regio's kunnen alleen thet behulp van grote investeringen in bv. Infrastructuur en scholing proberen de achterstand op de top regio's in te lopen.

Met betrekking tot de spreiding over tijd en regio vinden we dat kennis zich eerst verspreidt naar regio's dichtbij, en pas later naar regio's verder weg. Verder blijkt dat er na twee jaar een verzadigingspunt bereikt wordt waardoon kennis zich niet naar nog verder gelegen regio's verspreidt. Mogelijk wordt dit veroorzakakt door de lokale specificiteit en de veroudering van de kennis. 
Overigens blijkt dat de kennisoverdacht binnen een onderneming meer lokaal georienteerd is dan de kennisoverdracht tussen ondememingen. Wel zijn er duidelijke verschillen tussen de verschillende sectoren in onze databank. Gebaseerd op deze uitkomsten kunnen we stellen dat regionale innovatieve systemen nog steeds belangrijk zijn. Deze conclusie heeft ook grote politieke implicaties omdat het tot spanningen tussen regio's binnen de Europese markt kan leiden:

\section{Slot opmerkingen}

Voor ondernemingen die in de voorste gelederen van de economie willen meespelen is het dus erg belangrijk te werken aan hun absorberend vermogen door middel van investeringen in O\&O en door de vorming van allianties. Vetcler is het ook erg bellangrijk aanwezig te zijn in, of dichtbij, de regio's waat nieuwe technologische ontwikkelingen zich voordoen.

De kracht van dit proefschrift zit met name in de uitgebreidheid en nauwkeurigheid van de databank, waardoor het mogelijk is zaken te testen die voorheen niet testbaar waren. Door de wijze van constructie van de databank kunnen we ook zelfcitaties zoveel mogelijk tuit onze analyses filteren, wat de conclusies alleen mar sterker maakt. Ook kunnen we hierdoor de kennisstromen woot onze bedrijven opsplitsen in inter-en intra-multinationale kennisstromen. Verder beslat onze databank vrijwel alle multinationale ondernemingen die actief zijn in de hoog-en middeltechnologische sectoren van de economie. Onze onderzoeken, die gebaseerd zijn op Europese patentgegevens, zijn woorts een belangrijk complement voor de onderzoeken die zich baseren op Amerikaanse patent gegevens. Voor de Europese context warin onze onderzoeken plaatsvinden zijn de Europese patentgegevens mogelijk ook meer relevant dan de Amerikanse.

Patentgegevens hebben uiteraard hun beperkingen en onvolkomenheden (zie hoofdstuk2), naat yoot de meeste van deze problemen hebben we correcties doorgevoerd zodat we toch zinvolle conclusies hebben kunnen trekken op basis wan onze analyses. Verbeteringen die we zouden kumnen doorvoeren voor toekomstig onderzoek, hebben met name betrekking op het uirbreiden van de databank naar meerdere jaren en naar andersoortige bedrijven, met name kleinere bedrijven. Ook zou het goed zijn de relatie tussen de door ons gemeten kennisstromen en de winstgevendheid van de ondernemingen te onderzoeken. 


\section{Curriculum Vitae}

Wilfred Schoenmakers was born on the $12^{\text {th }}$ of May 1967 in Mastricht, The Netherlands. He received his secondary education at the Hentic wan Veldeke College in Maastricht. He studied Business economics at the Maastricht University with a specialisation in Marketing. Following his graduation in 1997 he started working as a tutor at the department of International Business Studies of the Maastricht University. In 1998 he started working on his Ph.D. project at the same department. During his Ph.D. project he followed courses at the Universite Louis pasteur in Strassbourg, the Erasmus University Rotterdam, the Technische Universiteit Einchoven and the University of Groningen. Currently he holds a Post-doc position at the Limburg University Centre in Diepenbeek (B).

Wilfred Schoenmakers werd op 12 mei 1967 geboren te Maastricht. Hij ronde achtereenvolgens de MAVO, de HAVO en het WWO aan het Henric van Veldeke College te Maastricht succesvol af. Hij studeerde aan de Universiteit Maastricht bedrijfseconomie met als specialisatie Marketing. In 1997 studeerde hij af en ging werken als docent bij de toenmalige sectie Internationale Bedrijfskunde aan de Universiteit Maastricht. Vanuit deze baan begon hij in 1998 als assistent-in-opleiding (aio) te werken aan zijn proefschrift bij dezelfde sectie. Tijdens zijn ailo-schap heeft hij diverse cursussen gevolgd o.a. aan de Université Louis pasteur in Strassbourg, de Erasmus Universiteit Rotterdam, de Technische Universiteit Eindhoven en de Rijksuniversiteit Groningen. Momenteel is hij als Post-doc verbonden aan het I.imburgs Universitair Centrum in Diepenbeek (B). 